NBS

Eechnical Note

\title{
THERMAL BALANCE IN THE F REGION OF THE ATMOSPHERE
}

\section{DOUGLAS C. HUNT}

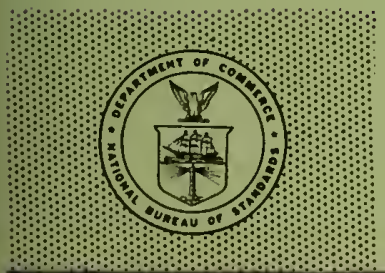

U. S. DEPARTMENT OF COMMERCE NATIONAL BUREAU OF STANDARDS 


\section{THE NATIONAL BUREAU OF STANDARDS}

\section{Functions and Activities}

The functions of the National Bureau of Standards are set forth in the Act of Congress, March 3, 1901, as amended by Congress in Public Law 619, 1950. These include the development and maintenance of the national standards of measurement and the provision of means and methods for making measurements consistent with these standards; the determination of physical constants and properties of materials; the development of methods and instruments for testing materials, devices, and structures; advisory services to government agencies on scientific and technical problems; invention and development of devices to serve special needs of the Government; and the development of standard practices, codes, and specifications. The work includes basic and applied research, development, engineering, instrumentation, testing, evaluation, calibration services, and various consultation and information services. Research projects are also performed for other government agencies when the work relates to and supplements the basic program of the Bureau or when the Bureau's unique competence is required. The scope of activities is suggested by the listing of divisions and sections on the inside of the back cover.

\section{Publications}

The results of the Bureau's research are published either in the Bureau's own series of publications or in the journals of professional and scientific societies. The Bureau itself publishes three periodicals available from the Government Printing Office: The Journal of Research, published in four separate sections, presents complete scientific and technical papers; the Technical News Bulletin presents summary and preliminary reports on work in progress; and Basic Radio Propagation Predictions provides data for determining the best frequencies to use for radio communications througho ut the world. There are also five series of nonperiodical publications: Monographs, Applied Mathematics Series, Handbooks, Miscellaneous Publications, and Technical Notes.

A complete listing of the Bureau's publications can be found in National Bureau of Standards Circular 460, Publications of the National Bureau of Standards, 1901 to June 1947 ( $\$ 1.25$ ), and the Supplement to National Bureau of Standards Circular 460, July 1947 to June 1957 (\$1.50), and Miscellaneous Publication 240, July 1957 to June 1960 (Includes Titles of Papers Publis hed in Outside Journals 1950 to 1959) (\$2.25); available from the Superintendent of Documents, Government Printing Office, Washington 25, D. C. 


\title{
NATIONAL BUREAU OF STANDARDS Eechnical Mote
}

162

\author{
SEPTEMBER 1962
}

\section{THERMAL BALANCE IN THE F REGION OF THE ATMOSPHERE}

\author{
Douglas C. Hunt \\ NBS Boulder Laboratories \\ Boulder, Colorado \\ (Central Radio Propagation Laboratory \\ and the University of Wyoming)
}

NBS Technical Notes are designed to supplement the Bureau's regular publications program. They provide a means for making available scientific data that are of transient or limited interest. Technical Notes may be listed or referred to in the open literature. 
CONTENTS

PAGE

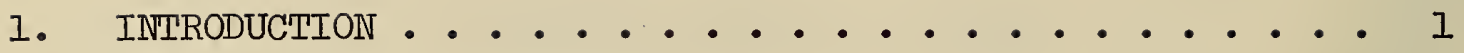

1.1 Scope of Present Work ............ I

1.2 Review of Atmospheric Physics ......... 1

1.3 Equilibrium Types in the Atmosphere ....... 3

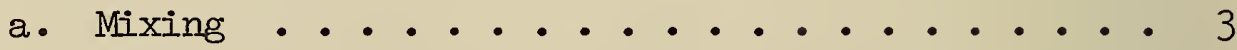

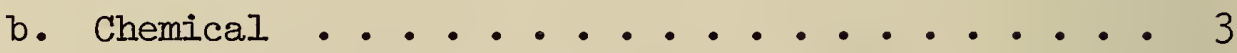

c. Diffusion ................. 4

2. THERMAL PROCESSES IN THE ATMOSPHERE $\ldots \ldots \ldots$

2.I The General Thermal Energy Continuity Equation ... 4

2.2 Terms in the Heat Balance Equation ...... 5

a. The Heat Conduction Term $\ldots . . . .5$

b. The Net Heat Generation Term ....... 6

c. The Heat Capacity Term ........ 9

3. DETAITED DISCUSSION OF THERMAL PROCESSES IN THE ASSUMFED

MODEL ...................... IO

3.1 The Gain Term, $G(h, t) \ldots \ldots 10$

3.2 The Conduction Term ........... 22

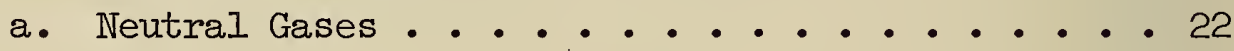

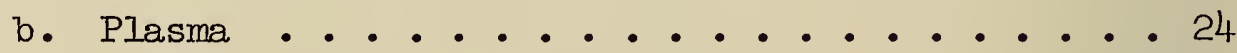

c. Turbulent Conduction . . . . . . . 25

3.3 The Ioss Term, $\mathrm{I}(\mathrm{h}, \mathrm{t}) \ldots \ldots . \ldots . . \ldots 25$

3.4 The Heat Capacity Term . . . . . . . 26 


\section{ABSTRACT}

An investigation is made of thermal mechanisms and detailed properties of the atmosphere at F-region heights. The method used constructs a thermal model of the atmospheric Fregion based on experimental data combined with theoretical results and arguments. The heat balance equation for this model is simultaneously solved with the barometric equation. The resultant mass density profiles are then compared with observed mass density profiles to determine how much heating by some extermal source is necessary in the Furégion.

Two cases are considered, one in which the time rate of change of temperature is zero (quasi-steady state) and one in which it is not zero (non-steady state). In the first case a complete solution is obtained. In the second case only a first approximation is carried out.

It is concluded that a heat flux of $1.13 \mathrm{ergs} / \mathrm{cm}^{2}-\mathrm{sec}$, originating in solar electromagnetic radiation, can satisfy the thermal requirements of the atmospheric F-region. The existence of other heat sources cannot be excluded altogether but their necessity is ruled out. For a 1.13 ergs/ $\mathrm{cm}^{2}-\mathrm{sec}$ electromagnetic radiation heat flux, a model of height and time variation of pertinent atmospheric parameters is constructed. These parameters are the temperature, molecular weight, scale height, detailed composition, optical depths, rate of heat generation due to absorption of solar electromagnetic radiation, rate of radiative heat loss, and the thermal conductivity. The effect of varying the parameters that determine the importance of the terms in the heat balance equation, and of varying the boundary conditions is tested. It is concluded that the uncertainties in these quantities cannot seriously affect the results obtained. 
4. SOLUTIONS OF EQUATIONS . . . . . . . . . . 27

4.I Terms in Final Form of Heat Balance Equation . . . 27

a. The Heat Capacity ......... 27

b. The Gain ............... 27

c. The Ioss ............ . . 28

d. The Conduction ............... 28

4.2 Boundary Conditions . . . . . . . . 29

a. The Height Range Considered . . . . . . 29

b. The Height $h_{d}$ and the Degree of $\mathrm{O}_{2}$ Dissociation - 29

c. The Temperature and the Total Mass Density at

$130 \mathrm{~km}$. . . . . . . . 30

d. The Conductive Flux at the Upper Boundary . . . 30

4.3 Solutions of the Heat Balance Equation ...... 30

a. Quasi-steady State Solution ........ 30

b. Non-steady State Solution . . . . . . 3 I

5. RESUITS AND DISCUSSION . . . . . . . . . 32

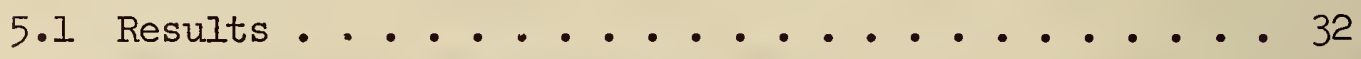

a. Quasi-steady State .......... 32

b. Non-steady State ............. 49

5.2 Boundary Conditions and Parameter Variation . . . 60

5.3 Range of Validity of Quasi-steady State Model . . . 70

5.4 The Non-steady State .......... 70

5.5 The Structure in the Observational Density and Scale

Height Profiles .......... 74

5.6 Conclusions . . . . . . . . . 75

5.7 Future Studies Indicated .......... 75

6. BIBLIOGRAPHY, .................... 76 


\section{FIGURES}

FIGURE

PAGE

1. $\partial \mathrm{T} / \partial \mathrm{t}$ necessary to make $\mathrm{C}_{\mathrm{V}} \rho \partial \mathrm{T} / \partial \mathrm{t}$ equal to the heat generation rate $\mathrm{G} . . . . . . . . . .11$

2. Temperature model for daytime and nighttime. . . . . 12

3. Solar electromagnetic flux outside the earth's atmosphere as a function of wavelength. ........ 16

4. Absorption cross sections of $\mathrm{O}_{2}$ versus wave length . . 19

5. Absorption cross sections of $\mathbb{N}_{2}$ versus wave length . . 20

6. Photoionization cross section of 0 versus wave length. . 21

7a. Mass densities versus height for heat flux triplets summing to $1.10 \mathrm{ergs} / \mathrm{cm}^{2}-\mathrm{sec}$............. 33

7b. Mass density versus height for heat flux triplets summing to $1.10 \mathrm{ergs} / \mathrm{cm}^{2}-\mathrm{sec} . . . . . . . . .34$

7c. Mass density versus height for heat flux triplets summing to $1.10 \mathrm{ergs} / \mathrm{cm}^{2}$-sec............... 35

8a. Scale heights versus height for heat flux triplets summing to $1.10 \mathrm{ergs} / \mathrm{cm}^{2}-\mathrm{sec} . . . . . . . . .36$

8b. Scale height versus height for heat flux triplets summing to $1.10 \mathrm{ergs} / \mathrm{cm}^{2}-\mathrm{sec} . . . . . . . . .37$

8c. Scale heights versus height for heat flux triplets summing to 1.10 ergs $/ \mathrm{cm}^{2}-\mathrm{sec} . . . . . . . . .38$

9. Scale heights versus height for several times $(x)$. The heat flux sum is 1.13 ergs $/ \mathrm{cm}^{2}-\mathrm{sec}$........ 39

10. Mass densities versus height for several times $(x)$. The heat flux sum is $1.13 \mathrm{ergs} / \mathrm{cm}^{2}-\mathrm{sec} . . . . . .40$

11. Temperature versus height for several times $(x)$. The heat flux sum is $1.13 \mathrm{ergs} / \mathrm{cm}^{2}-\mathrm{sec}$........ 42 
12. Mass densities versus time $(x)$ for several heights. The heat flux sum is 1.13 ergs $/ \mathrm{cm}^{2}-\mathrm{sec} . . . . . . .43$

13. Temperature versus time $(x)$ for several heights. The heat flux sum is 1.13 ergs $/ \mathrm{cm}^{2}-\mathrm{sec}$......... 44

14. Detailed composition versus height for a heat flux sum of 1.13 ergs $/ \mathrm{cm}^{2}-\mathrm{sec}$ and $x=0^{\circ}$........... 45

15. Detailed composition versus height for a heat flux sum of $1.13 \mathrm{ergs} / \mathrm{cm}^{2}-\mathrm{sec}$ and $x=30^{\circ}$.......... 46

16. Detailed composition versus height for a heat flux sum of 1.13 ergs $/ \mathrm{cm}^{2}-\mathrm{sec}$ and $x=60^{\circ} \ldots . . . . . .47$

17. Detailed composition versus height for a heat flux sum of 1.13 ergs $/ \mathrm{cm}^{2}-\mathrm{sec}$ and $x=90^{\circ}$......... 48

18. Optical depths versus height at several times $(x)$ for $584 \mathrm{~A}$ wave length range. The total heat flux is 1.13

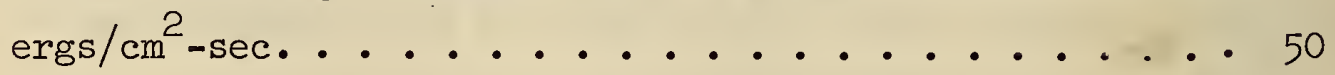

19. Optical depths versus height for $900 \mathrm{~A}$ wave length range at several times $(x)$. The total heat flux is

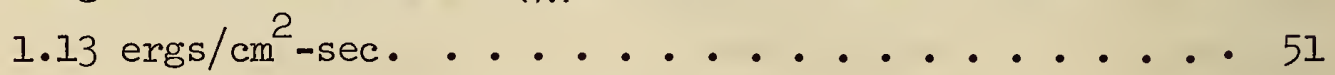

20. Optical depths versus height for several times $(x)$ in the $304 \mathrm{~A}$ wave length range. The total heat flux is

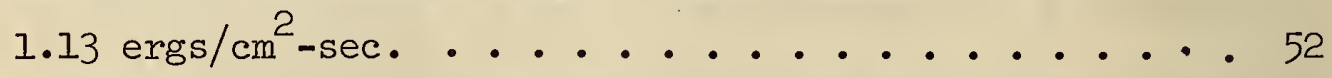

21. Rate of heat generation versus height for several times $(x)$. The total heat flux is $1.25 \mathrm{ergs} / \mathrm{cm}^{2}-\mathrm{sec} . . .53$

22. Radiative heat loss rate versus height for several times $(x)$ and a total heat flux of $1.25 \mathrm{ergs} / \mathrm{cm}^{2}-\mathrm{sec} . . .54$

23. Molecular weight and thermal conductivity versus height for a total heat flux of $1.25 \mathrm{ergs} / \mathrm{cm}^{2}$ at $x=0^{\circ}$... 55 24. Non-steady state mass densities versus height for several times $(x)$ and a total heat flux of $1.0 \mathrm{ergs} / \mathrm{cm}^{2}-\mathrm{sec} . .56$ 
25. Non-steady state temperatures versus height for several times $(x)$. The total heat flux is $1.00 \mathrm{erg} / \mathrm{cm}^{2}-\mathrm{sec} . . .57$

26. Non-steady state mass densities versus time $(x)$ for several heights and a total heat flux of $1.00 \mathrm{erg} / \mathrm{cm}^{2}-\mathrm{sec} \cdot .58$

27. Non-steady state temperatures versus time $(x)$ for several heights and a total heat flux of $1.00 \mathrm{erg} / \mathrm{cm}^{2}-\mathrm{sec} . . . \quad 59$

28. Effect of varying $H(130)$ by varying $T(130)$ for a total heat flux of 1.10 ergs $/ \mathrm{cm}^{2}-\mathrm{sec}$. . . . . . . . . 61

29. Effect of varying $H(130)$ by varying $M(130)$ for a total

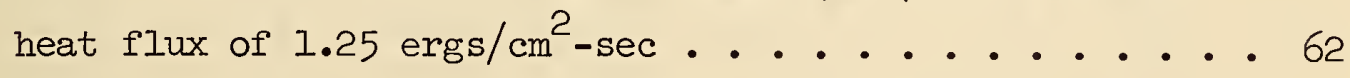

30. Effect of varying boundary mass density. The total heat flux is $1.25 \mathrm{ergs} / \mathrm{cm}^{2}-\mathrm{sec}$ and $\dot{x}$ is $0^{\circ}$......... 63

31. Effect on $\rho(h)$ of varying cross sections for a total heat flux of $1.25 \mathrm{ergs} / \mathrm{cm}^{2}-\mathrm{sec}$. . . . . . . . . . . 64

32. Effect of varying $h_{\mathrm{d}}$ for a total heat flux of 1.10 ergs $/ \mathrm{cm}^{2}-\sec$ at $x=0$. . . . . . . . . . . . 66

33. Effect of varying total heat flux. . . . . . . . . 67

34. Effect of varying thermal conductivity for a total heat flux of $1.10 \mathrm{ergs} / \mathrm{cm}^{2}-\mathrm{sec}$ at $x=0^{\circ}$.......... 68

35. The effect of varying the conductive thermal flux at the upper boundary. The total heat flux is $1.10 \mathrm{ergs} / \mathrm{cm}^{2}-\mathrm{sec}$ at $a \times$ of $0^{\circ} \cdot$. . . . . . . . . . . . . 69

36. Effect of varying compositions on mass density profiles for a total heat flux of $1.10 \mathrm{ergs} / \mathrm{cm}^{2}-\sec \left(x=0^{\circ}\right)$. . 71

37. Effect on composition and total mass density of Blamont, et. al. boundary conditions for a total heat flux of 1.25 ergs $/ \mathrm{cm}^{2}-\mathrm{sec}$ and $x=0$. Model curve is computed for boundary conditions selected in paper 

THERMAL BALANCE IN THE F REGION OF THE ATMOSPHERE

\section{Douglas C. Hunt}

\section{INTRODUCTION}

\subsection{The Scope of the Present Work}

The primary properties of an atmosphere are its density, temperature, pressure, composition, and motion. With the advent of high altitude rockets and globe-circling satellites, these properties are becoming better known up to very great heights in the Earth's atmosphere. However, there is still considerable uncertainty in these properties at F-region and upper E-region heights (about 130-500 km). Mass densities are here known to within a factor of two and from these pressures of about the same accuracy may be computed. However, the detailed properties, the temperature, composition, and motion are poorly known. This study makes use of the thermal balance in the F-region to investigate the atmospheric temperature and density. First, the mechanisms which contribute to the thermal balance are investigated both on the basis of experimental data and theoretical arguments. Then a thermal model is constructed (using photoionization by solar electromagnetic radiation as the F-region's primary heating source) which predicts the observed F-region daytime mass density profiles very closely. Finally, a daytime model of the detailed atmospheric properties, giving their height and time variation, is derived.

\subsection{Revièw of Atmospheric Physics}

If there is no relative velocity between the motion of the center of masses of the various constituents of a gas, no net motion of the total center of mass of the gas, and if thermal diffusion can be neglected (conditions which usually prevail in the atmosphere), then the gas is in hydrostatic equilibrium. In the atmosphere where the applied force is primarily that of gravity, the equation of hydrostatic equilibrium may then be used as follows:

$$
\mathrm{dp}(\mathrm{h}) / \mathrm{dh}=-\rho(\mathrm{h}) \mathrm{g}(\mathrm{h}),
$$

where $h$ is the height relative to the surface of the earth, $p(h)$ is the hydrostatic pressure, $g(h)$ is the acceleration of gravity, and $\rho(h)$ is the mass density of the gas. If the above equilibrium conditions do not hold (which may happen for a short while around sunrise and sunset), the continuity equation for momentum must be applied.

The mass density of the $i^{\text {th }}$ atmospheric constituent, $\rho_{i}(h)$, is related to the number density of the $i^{\text {th }}$ constituent, $n_{i}(h)$, by:

$$
\rho_{i}(h)=n_{i}(h) M_{i}(h) / N_{0}
$$


Here $M_{i}(h)$ is the gram molecular weight of the $i^{\text {th }}$ constituent and $N_{0}=$ $6.02 \times 10^{23}$ particles/mole is Avogadro's number. If by analogy then $\rho(h)$ is given as follows:

$$
\rho(h)=n(h) M(h) / N_{0} \text {, }
$$

where $\rho(h)$ is the total mass density, $n(h)$ is the total number density, and $M(h)$ is the mean gram molecular weight, then (1-2) and (1-3) yield:

$$
\rho(h) \equiv \sum_{i} \rho_{i}=\sum_{i} n_{i}(h) M_{i} / N_{0}=n(h) M(h) / N_{0}
$$

giving

$$
M(h)=\sum_{i} n_{i}(h) M_{i} / \sum_{i} n_{i}(h)
$$

where

$$
n(h) \equiv \sum_{i} n_{i}(h)
$$

In the upper atmosphere, because of the low pressure, the gas molecules behave in their interactions with each other almost exactly as elastic rigid spheres. Thus the ideal gas law is applicable and is written:

$$
p(h)=n(h) k T(h)
$$

Here $\mathrm{T}(\mathrm{h})$ is the kinetic temperature and $\mathrm{k}=1.38 \times 10^{-16} \mathrm{ergs} /{ }^{\circ} \mathrm{K}$ is Boltzmann's constant. Dividing (1-l) through by $p(h)$ and using (1-6) yields:

$$
\mathrm{d} \operatorname{lnp}(\mathrm{h}) / \mathrm{dh}=-\mathrm{I} / \mathrm{H}(\mathrm{h})
$$

Here $H(h)=R T(h) / M(h) g(h)$ and $R=N_{0} k=8.314 \times 10^{7}$ ergs $/$ mole $-K$ is the gas constant. Integrating ( $1-7)$ from some low height, $h_{0}$, to $h$ then gives: 


$$
p(h)=p\left(h_{0}\right) \exp \left(-\int_{h}^{h} \frac{d h}{H(h)}\right) .
$$

Here $H(h)$ is normally termed the "scale height" and physically is the logarithmic decrement of the pressure. Then using (1-3) and (1-6) gives what will be called the "barometric equation."

$$
\rho(h)=\frac{\rho\left(h_{0}\right) T\left(h_{0}\right) M(h)}{M\left(h_{0}\right) T(h)} \exp \left(-\int_{h_{0}}^{h} \frac{d h}{H(h)}\right) .
$$

\subsection{Equilibrium Types in the Atmosphere}

In order to know the detailed composition of the atmosphere one must know what process is dominant in its equilibrium behavi.or. The following are important in the atmosphere.

\section{a. Mixing}

If there are strong instabilities or turbulences present, the atmosphere may be forced to maintain the same percentage composition over a height range. Except for some trace constituents, observations of composition indicate the atmosphere to be mixed up to the mesopause (about $85 \mathrm{~km}$ ). The reasons for the instabilities leading to mixing are not well understood above the tropopause $(10-15 \mathrm{~km})$. It is possible that they may be caused by disturbances originating in the troposphere and propagated upward to be dissipated above the tropopause.

\section{b. Chemical}

If a constituent is being produced or destroyed by some reaction, this may be the controlling factor in the constituent's height distribution. The distribution of ozone, $\mathrm{O}_{3}$, in the stratosphere is an example of this (Wulf and Deming, 1937). Even though the atmosphere is generally turbulent in the stratosphere, the chemical reactions involving $\mathrm{O}_{3}$ are rapid enough to produce an $\mathrm{O}_{3}$ layer. Above the mesopause the dissociation of molecular oxygen, $\mathrm{O}_{2}$, starts to occur and soon becomes so rapid that turbulence cannot keep up with it. Early theories of $\mathrm{O}_{2}$ dissociation (Penndorf, 1949) assumed complete photochemical equilibrium, but more recent work (Nicolet and Mange, 1954) has shown that such an equilibrium cannot adequately explain this problem. 
If the times for establishing turbulent or chemical equilibrium are long compared with the diffusive time constant (the diffusive time constant is about 12 hours at $120 \mathrm{~km}$, and decreases exponentially above this height), then each constituent will tend to arrange itself according to its molecular weight. The heavier ones will fall and the lighter ones will rise. Such an equilibrium is termed diffusive. In this case the law of partial pressures may be applied and (1-1) and (1-6) may be written for each constituent. They are then combined as before giving the barometric equation for each constituent.

$$
\rho_{i}(h)=\frac{\rho_{i}\left(h_{0}\right) T\left(h_{0}\right)}{T(h)} \exp \left[-\int_{h_{0}}^{h} \frac{d h}{H_{i}(h)}\right] .
$$

Here $\mathrm{H}_{i}(\mathrm{~h})=\mathrm{RT}(\mathrm{h}) / \mathrm{M}_{i} \mathrm{~g}(\mathrm{~h})$.

The exact height above which diffusion predominates is not precisely known. Afternoon rocket observations (Meadows and Townsend, 1960) indicate a diffusive equilibrium between argon, $A$, and molecular nitrogen, $\mathbb{N}_{2}$, to start between 100 and $120 \mathrm{~km}$, indicating that the total atmosphere should be diffusively controlled above these heights. Nicolet (1960) asserts that, because of the increase of pressure when diffusion replaces mixing, diffusion may be inhibited until 150 to $160 \mathrm{~km}$. The height of the start of diffusive ecuilibrium, $h_{d}$, will probably be a function of time and will likely be highest during midday. This is because atmospheric chemistry and turbulence are related to the diurnal variation of heat flux absorption.

\section{THERMAL PROCESSES IN THE ATMOSPHERE}

\section{I The General Thermal Energy Continuity Equation}

The thermal balance in a gas is controlled by what will be called the thermal energy continuity equation. In the thermal energy continuity equation the rate at which the thermal energy of a unit volume of a gas changes is due to the following causes:

\section{a. Particle Divergence}

This energy change is due to a net influx or outflow of molecules. This net flow may be due to vertical transport (mutual diffusion of one constituent with respect to another in a several constituent gas) or convection. 


\section{b. Energy Divergence}

This energy change is due to different energies of the inflowing and outflowing molecules rather than different numbers. This flow is that due to ordinary thermal conduction.

\section{c. Surface Pressures}

This energy change is due to the work done by the surface pressures on the unit volume of gas.

In the atmospheric $F$ region, only the energy change due to vertical thermal conduction is assumed to be important. Also, to include the effects of sources and sinks of thermal energy, a net rate of heat generation (per unit volume) term, $G(h, t) \equiv G$, is included in the thermal energy continuity equation. The thermal energy continuity equation is then written in the following form, which will be referred to as the heat balance equation:

$$
\left.C_{V} \rho \partial T / \partial t=-\vec{\nabla} \cdot \vec{q}\right)_{z}+G
$$

Here $C_{V}$ is the specific heat of the gas at constant volume and $\left.\vec{\nabla} \cdot \vec{q}\right)_{z}$ is the derivative with respect to the vertical direction of the conductive heat flux vector, $\vec{q}$.

The neglect of the other terms is done, of course, to make the final equation more tractable and because such a neglect is a good approximation. However, any terms that do not add dimensions (time or space) to the problem may be included if a model of the functions involved is available.

\subsection{Terms in the Heat Balance Equation}

$$
\text { a. The Heat Conduction Term } \vec{\nabla} \cdot \vec{q})_{z}
$$

For a binary gas mixture, the heat flux vector, $\vec{q}$, will consist of three parts:

$$
\begin{aligned}
q_{z} & =\lambda \partial T / \partial h+5 / 2 k T\left(n_{1} \bar{C}_{1 z}+n_{2} \bar{C}_{2 z}\right) \\
& +\operatorname{knTk}_{T T}\left(\bar{C}_{1 z}-\bar{C}_{2 z}\right)
\end{aligned}
$$


where $\lambda$ is the thermal conductivity. The first term is the ordinary heat flow due to temperature inequalities in the gas. The second and third terms occur because of the relative diffusion of the two gases. The second term is relatively unimportant since it is the flow due to the fact that the thermal flux is measured relative to the mass velocity $\overrightarrow{\mathrm{c}}_{0}$ and not to $\overrightarrow{\vec{c}}$ (the mean molecular velocity). It may thus be transformed away. It this is done only the factor, $\mathrm{knTk}_{\mathrm{T}} \mathrm{C}_{1 z}$, remains where now $\mathrm{C}_{1 \mathrm{z}}$ is measured with respect to $\overrightarrow{\overrightarrow{\mathrm{c}}}$. This is the actual heat flow due to diffusion. This heat flow is a transient one which will disappear when mutual diffusion has stopped. Also, calculations of the ordinary conduction term compared with the diffusion term, based on average Fregion parameters (Champion, Minzner and Pond, 1959 ; Chapman and Cowling, 1952), indicate that a value of $\mathrm{C}_{1 \mathrm{z}}$ of about $100 \mathrm{~m} / \mathrm{sec}$ is necessary to make the diffusion term comparable in magnitude to the ordinary conduction term. Based on this and the previously postulated transport model, the thermal flux is taken to be due only to ordinary conduction.

\section{b. The Net Heat Generation Term}

This term includes sources and sinks of heat in the F-region. It will be taken to consist of three terms as follows:

$$
G=G(h, t)-L(h, t)+G_{c}(h, t) .
$$

The three terms in (2-2) will be discussed separately.

(l) The local loss term, $L(h, t)$. This term includes whatever thermal energy is lost to the atmospheric particles due to "in situ" processes such as radiation from collisionally de-excited particles or particles excited due to chemical reactions.

(2) The convective transfer term, $G_{c}(h, t)$. This term includes the net heat gain or loss due to the influx or outflow of molecules which have been heated or cooled elsewhere. This term has been neglected primarily because there is insufficient information available about it to include it intelligently. However, the data which do exist indicate that horizontal pressure gradients are probably insufficient to cause appreciable convective transfer. Johnson (1960) has suggested that convective transfer may be important in the vicinity of $200 \mathrm{~km}$; this possibility is treated further in the discussion of results.

(3) The local gain term, $G(h, t)$. This term includes all of the possible "in site" methods of heat generation. Many have been suggested 
by various workers at one time or another. Some can empirically or theoretically be argued to be small. A list of these with some argument as to their relative importance follows:

(a) Photonic heating: The possibility of heating the F-region by solar electromagnetic radiation depends on several things. First, there must be a certain solar wavelength range in which the probability of interaction with the F-region constituents is high. If there is such a wavelength range there must be enough photons in it to cause appreciable interaction. Iastly, there must be a way to convert efficiently the interaction energy into thermal energy of the molecules and atoms of the F region. Such a wavelength range is in fact available in the far ultraviolet region of the solar spectrum. In the range from about 170-912 A the interation probability (the absorption cross section ) is large $\left(\approx 10^{-17}-10^{-18} \mathrm{~cm}^{2}\right)$. Also rocket measurements (Violette and Rense, 1959; Hinteregger, et al., 1960) show the photon flux in this range to be on the order of $5 \mathrm{ergs} / \mathrm{cm}^{2}-\mathrm{sec}$. A theoretical estimate of the amount of absorbed energy turned into thermal energy (Hanson and Johnson, 1961) give a "thermal efficiency," $\epsilon(h, \lambda)$, for 304 A of $0.15-0.30$. This would mean that from $0.75-1.50 \mathrm{ergs} / \mathrm{cm}^{2}-\mathrm{sec}$ of thermal energy would be made available to the F-region if all the F-region ionizing flux were in the $304 \mathrm{~A}$ line. However, there is considerable flux in other interacting wavelength ranges. Thus, since the amount of thermal energy available per photon is roughly independent of photon energy (Hanson and Johnson, 1961), the amount of available thermal energy should be higher than the indicated range.

(b) Dissipation of hydromagnetic waves: This mechanism as a source of atmospheric heating was suggested by Dessler (1959). According to Dessler, interaction of the so-called "solar wind" with the Earth's static magnetic field may cause instabilities which generate electromagnetic waves. The magnetic component of these waves adds to the Earth's static field giving a time-varying magnetic field which is called a hydromagnetic wave. Ions in the atmosphere tend to follow the hydromagnetic wave and their extra kinetic energy may then collisionally heat the atmosphere. Dessler estimates the dissipation to peak at about $175 \mathrm{~km}$ with a maximum dissipative value of $5 \times 10^{-8} \mathrm{ergs} / \mathrm{cm}^{3}-\mathrm{sec}$. This would imply a total heat flux of about $0.7 \mathrm{ergs} / \mathrm{cm}^{2}-\mathrm{sec}$. This must, however, be regarded as a very rough estimate because the amplitudes of the hydromagnetic waves are not well established. Dessler also notes that during magnetic storms the amplitude of the waves may increase by a factor of 100, causing hydromagnetic waves to be a significant heat source. Some evidence for the existence of hydromagnetic waves exists, (Sugiura, 1961), but it is still generally thought to be a relatively minor heat source. Its height profile of heat generation is quite similar to that of photonic heating and so would be hard to distinguish from photonic heating. 
(c) Corpuscular heating: This has been suggested (Krassovsky, 1960) as the primary F-region heating source. Krassovsky obtains, from Sputnik III data, electron energy fluxes of about $100 \mathrm{ergs} / \mathrm{cm}^{2}-\mathrm{sec}$ at $1720-1880 \mathrm{~km}$, and estimates that about $1 \mathrm{erg} / \mathrm{cm}^{2}-\mathrm{sec}$ of this can reach the F-region. Because of the relatively long mean free path and short radius of gyration of these particles in the Earth's magnetic field at these heights ( $>1000 \mathrm{~km}$ ), the chances of an energy transferring collision before the particle is thrown back out into space are quite small. At the poles, however, the magnetic field cannot as effectively turn back these particles and hence, the occurrence of auroras. Bright auroras may involve energy fluxes of as much as $2000 \mathrm{ergs} / \mathrm{cm}^{2}-\mathrm{sec}$ (McIlwain, 1960). Some resultant local heating may occur, but the infrequency of strong auroras and the small fraction of the Earth's surface which they cover, indicate global effects are quite small. If corpuscular heating occurs, its profile should resemble that of photonic heating and would also be difficult to distinguish from the first two heating sources.

(d) Coronal conduction: This mechanism originally suggested by Chapman (1951), should be closely related to corpuscular heating. It is the conduction of heat to a cool speck (the Earth) imbedded in the hot gas of the Sun's corona. Chapman estimates a heat flux of about $0.2 \mathrm{ergs} / \mathrm{cm}^{2}$-sec may be brought into the atmosphere by this means. Again, because of the Earth's magnetic field, the conducting particles should enter the atmosphere mainly in the polar regions and give very little energy to the temperate latitudes before being thrown back into space. Also, as pointed out before, such a flux is unlikely to lead to appreciable global effects. This heating mechanism should be distinguishable from the true "in situ" heat sources.

(e) Meteorites: Johnson (1958), in his paper on atmospheric heating, has estimated from rocket data that the heating rate by micrometeorites would be $0.1^{\circ} \mathrm{K} /$ day. This would be caused by meteors of average mass $10^{-12} \mathrm{gm}$ and velocity of about $12 \mathrm{~km} / \mathrm{sec}$, statistics which Johnson says fits current meteoric theories best. In general, meteorites are thought to expend most of their energy in the E- and upper D-regions of the atmosphere. Kaiser (1955) has estimates that sporadic meteorites should produce about $2.3 \times 10^{-4}$ electrons $/ \mathrm{cm}^{3}-\mathrm{sec}$ in the E-region. If a thermal input of $6 \mathrm{e} \cdot \mathrm{v} \cdot /$ ionization is effected, that the total meteoric heat flux would be about $10^{-8} \mathrm{ergs} / \mathrm{cm}^{2}-\mathrm{sec}$. During meteor showers this value may rise considerably. Lovell (1950) has estimated that the ionization rate may then be $3 \times 10^{3}$ electrons $/ \mathrm{cm}^{3}-\mathrm{sec}$, leading to a meteoric heat flux of about $0.05 \mathrm{ergs} / \mathrm{cm}^{2}-\mathrm{sec}$. Thus it is seen that meteorites are almost certainly never an important F-region heat source. 
(f) Joule heating: Krassovsky (1959) has proposed that atmospheric heating may be due to currents induced by magnetic fields imprisoned by solar corpuscular streams incident upon the Earth. This theory has not been developed in detail, but as Bates (1959) points out, one unattractive feature of it is the fact that magnetic field energy of $10^{22}$ ergs must be changed into upper atmospheric thermal energy each hour. This is of the order of the field energy occurring during a magnetic storm. This then suggests that, as is the case with hydromagnetic heating, such a mechanism could be significant only during magnetic storms.

(g) Dissipation of infrasonic waves: Daniels (1952) has proposed a theory based on acoustical waves generated by the ocean. He estimates the average radiated acoustical energy to be $100 \mathrm{ergs} / \mathrm{cm}^{2}-\mathrm{sec}$. Under isothermal conditions and if $\epsilon$ is unity for this process, he concludes all this energy would be absorbed between 130 and $155 \mathrm{~km}$ and could heat the F-region by $300^{\circ} \mathrm{K} / \mathrm{hr}$. The absence of isothermality, the presence of temperature inversions, and winds in the $0-100 \mathrm{~km}$ range render the transport of such waves rather uncertain. Also, $\epsilon$ for this process will probably be considerably less than unity. These reasons, plus the relatively uncertain method by which the acoustical energy radiation rate was derived, make this mechanism a doubtful heat source.

To summarize, while there are a number of ways in which the temperate latitude F-region may be heated, the only one which is well established both theoretically and experimentally as a strong thermal energy source is heating by solar electromagnetic radiation. It will, therefore, be assumed that the F-region is heated entirely by a solar photon flux.

\section{c. The Heat Capacity Term $c_{v} p \partial T / \partial t$}

This term adds the extra dimension, time, to the total differential equation represented by the right hand side of the heat balance equation. When this term is negligible compared to the terms on the right side of the heat balance equation, the atmosphere will be said to be in the quasisteady state due to the fact that the G term may be time dependent. It is appropriate, therefore, to investigate the conditions under which it is possible to neglect the heat capacity term. Heating by solar electromagnetic radiation, which has been selected as the F-region's heating source, has a strong diurnal variation. Thus the general form of the diurnal temperature variation is the following: in the morning there will be a temperature rise. The rise will continue until sometime in the aftemoon and then will decline somewhat until sunset at the considered height. A decline in the temperature will then occur, rather rapidly at first, until a minimum is reached sometime early in the morning. Thus at two times, near sunrise and sometime during the day (pro- 
bably in the early afternoon), $\partial \mathrm{T} / \partial t \equiv \mathrm{T}$ will be zero, implying the quasi-steady state to be valid for at least a short time around these times and at the height in question. Now to obtain a rough quantitative idea of the importance of the heat capacity term, values of $T$ necessary to make this term equal to $G$ are plotted in figure 1 . These T's are obtained by using the expression, $|T|=|[G(h, t)-L(h, t)]| / c_{v} \rho(h, t)$. The $\rho(h, t)$ are taken from a daytime mass density profile (Kallman, 196l). The daytime density profile gives densities near a time when $T \approx 0$ at all heights so that the values of $G(h, t)-I(h, t)$ used to compute the $\dot{T}$ 's are taken from computations made for noon assuming the quasi-steady state to hold: Since the ratio of $G$ to $p(h, t)$ is roughly constant in time, these $|\dot{T}|$ 's will be representative of the entire day. Approximate temperature profiles are plotted for noon and 0800 hours, assuming the quasi-steady state to hold (figure 2). These are thought to approximate the maximum daytime and minimum nighttime temperature profiles. Using these curves, and assuming most of the day-to-night temperature changes occur in a six hour period, the following table of average $|\dot{T}|$ 's is computed:
$\mathrm{h}(\mathrm{km})$
150
200
300
400
500
$|\dot{\mathrm{T}}|\left({ }^{\circ} \mathrm{K} / \mathrm{hr}\right)$
26
53
80
87
91

where $h$ is the height above the Earth's surface. These numbers should now be compared with $|\dot{T}|$ 's (figure 1 ).

From these considerations it is seen that, on the average, the heat capacity term during the day will be only about ten percent of $G(h, t)$ above approximately $170 \mathrm{~km}$. It is concluded that, except at low Fregion heights, soon after sunrise, and around sunset, the daytime Fregion atmosphere is represented approximately by the quasi-steady state.

\section{DETAILED DISCUSSION OF THERMAI PROCESSES}

IN THE ASSUMED MODEL

\section{I The Gain Term, $G(h, t)$}

The attenuation of a photon beam may be accomplished through photoionization, photodissociation, resonance or fluorescent scattering, Compton scattering by free (modified line) or bound (unmodified line) electrons, or through pair production. Since this absorption is a statistical process the beam attenuation will be proportional both to the beam intensity and to the incremental path length traversed. Thus for a monoenergetic beam we may write: 
11

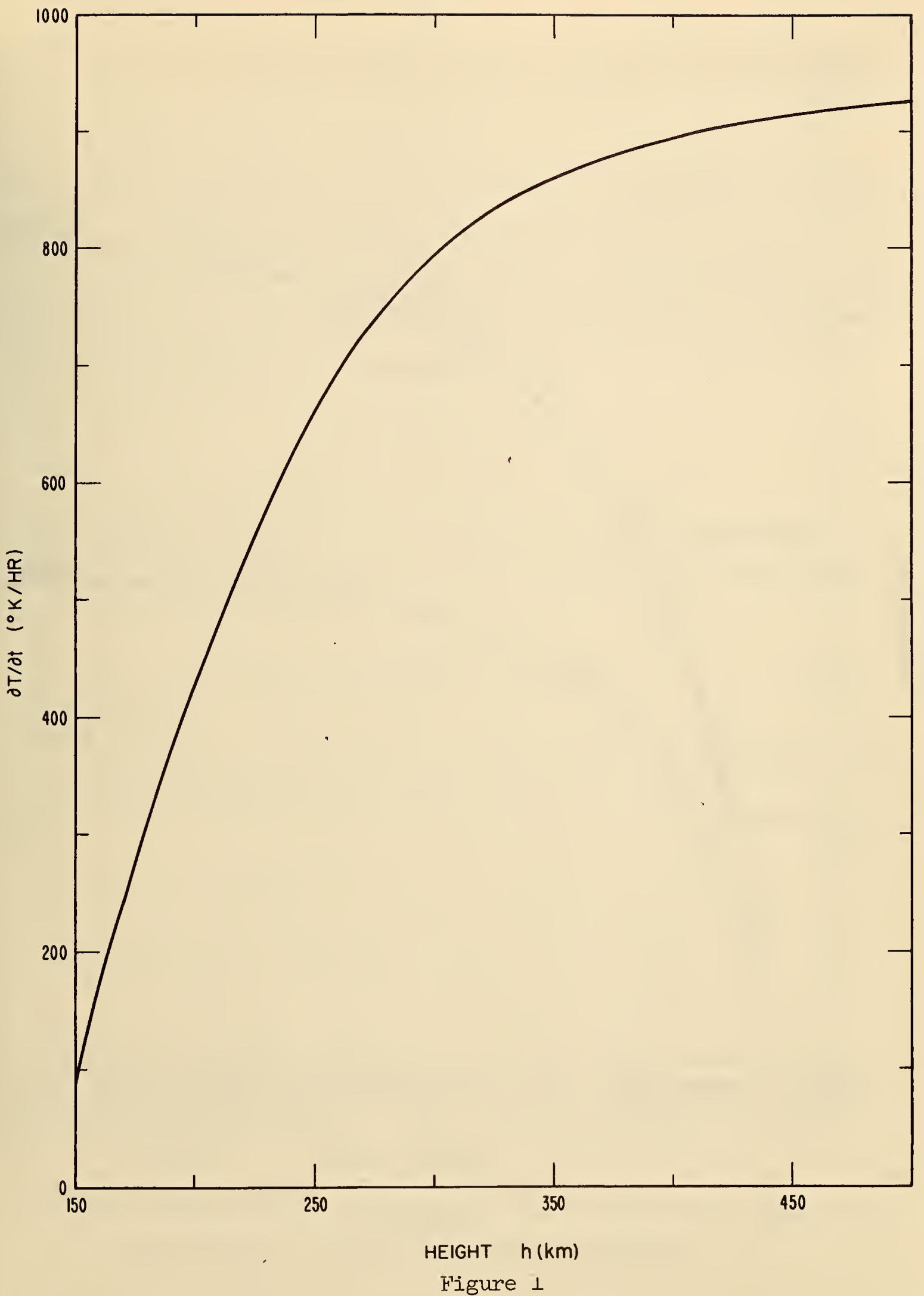

F'igure 1

$\partial \mathrm{T} / \partial t$ Necessary to Make $\mathrm{C}_{\mathrm{v}} \mathrm{p \partial T} / \partial t$

Equal to the Heat Generation Rate G 


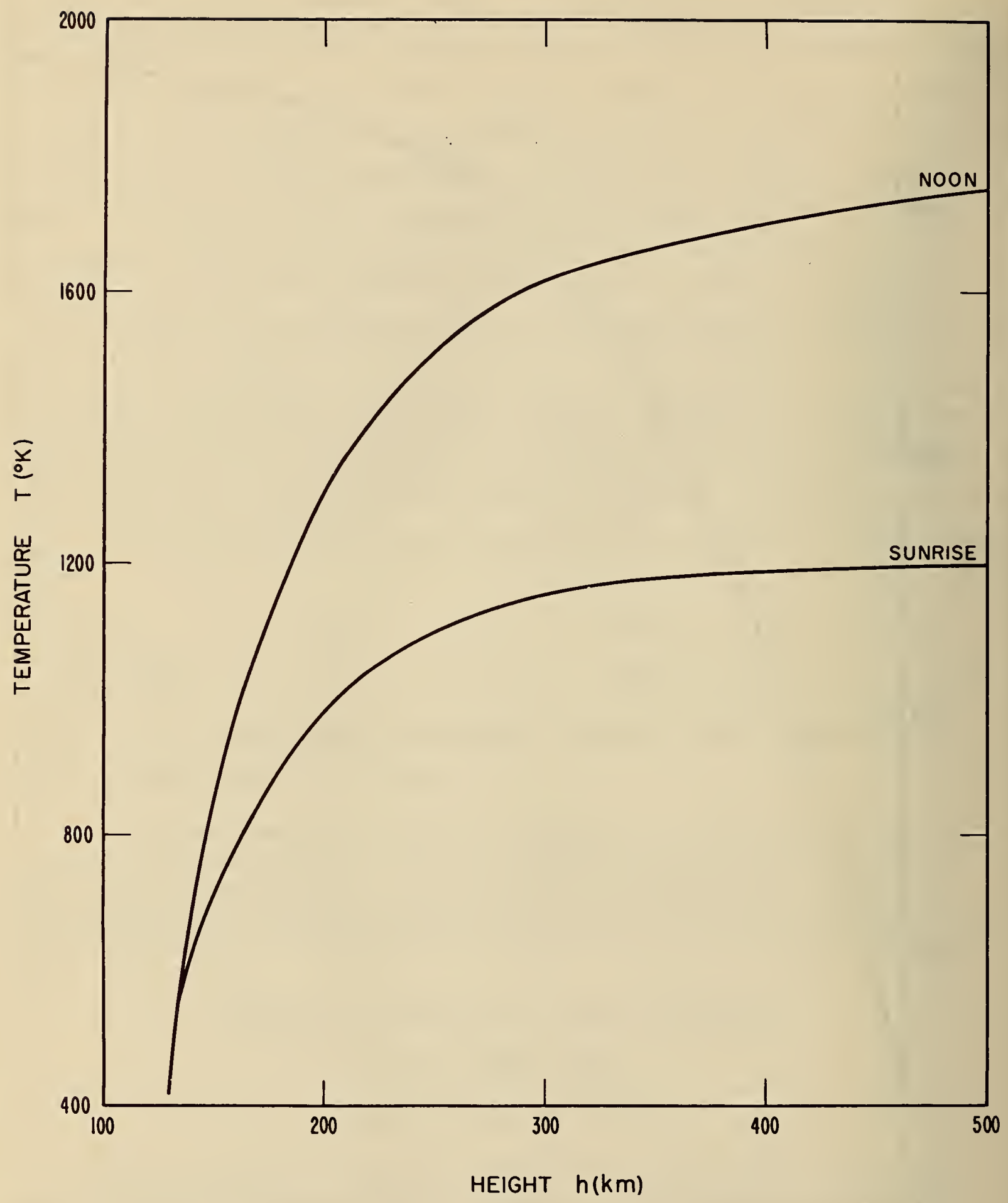

Figure 2

Temperature Model for Daytime and Nighttime 


$$
d I(\lambda, h)=-\sum_{i} \mu_{i}(\lambda, h) I(\lambda, h) d s
$$

where the negative sign implies absorption. Here $I(\lambda, h)$ is the beam intensity per unit wavelength, $\mu_{i}(\lambda, h)$ is the linear absorption coefficient tor the $i^{\text {th }}$ atmospheric constituent, and $d s$ is the path length traversed. For purposes of atmospheric absorption a plane parallel atmosphere will first be assumed in which $\mathrm{d} s=\sec x \mathrm{dh}$, where $x$ is the solar zenith angle. Also, Beer's law will be assumed to hold; it states that the proportionality constant between $\mu_{i}$ and the absorber concentration is a constant independent of temperature and pressure. Thus:

$$
\mu_{i}(\lambda, h)=A_{i}(\lambda) p_{i}(h)
$$

Here $A_{i}(\lambda)$ is the absorption cross section for the $i^{\text {th }}$ atmospheric constituent in $\mathrm{cm}^{2} / \mathrm{gm}$. Equations $(3-1)$ and (3-2) then upon integration give, using the ds definition:

$$
I(\lambda, h)=I_{\infty}(\lambda) \exp [-\tau(\lambda, h) \sec \chi]
$$

Here $T(\lambda, h) \equiv \sum_{i} \int_{h}^{\infty} A_{i}(\lambda) \rho_{i}(h) d h$ is the optical depth for wavelength $\lambda$ and $I_{\infty}(\lambda)$ is the beam intensity per unit wavelength outside the Earth's atmosphere.

The rate of ionization or the rate of heat generation is given by:

$$
G(\lambda, h)=\epsilon(\lambda, h) d I(\lambda, h) / d s=\epsilon(\lambda, h) \cos \chi d I(\lambda, h) / d h
$$

Here $\epsilon(\lambda, h)$ is the quantum efficiency or number of electrons produced per unit energy increment absorbed (in the case of ionization) or the amount of thermal energy produced per unit energy increment absorbed (in the case of heat generation). The latter meaning will always be assumed in this paper. by:

The total heat generation rate due to all wavelengths is then given

$$
G(h, x)=\int_{0}^{\infty} G(\lambda, h) d \lambda \equiv G(h, t)
$$


Equations (3-3), (3-4), and (3-5) then give the total heat generation rate, $G(h, t)$, as:

$$
G(h, t)=\int_{0}^{\infty} \epsilon(\lambda, h) I_{\infty}(\lambda) \sum_{i} A_{i}(\lambda) \rho_{i}(h) \exp [-\tau(\lambda, h) \sec x] d \lambda .
$$

The terms in (3-6) will now be considered separately.

$$
\text { a. } \rho_{i}(h)
$$

As mentioned before, $h_{d}$ probably lies in the upper E-region. Since $\mathrm{N}_{2}$ is very stable against photodissociation, while $\mathrm{O}_{2}$ may be dissociated, it is likely that there is a considerable amount of $\mathrm{N}_{2}$ and atomic oxygen, 0 , present. In diffusive equilibrium the lighter constituents persist to higher heights so that even though $\mathrm{N}_{2}$ will predominate at $h_{d}$, 0 will become dominant in the upper F-region. It is fairly certain that at $h_{d}$, $\mathrm{O}_{2}$ will be considerably dissociated (Kupperian, et al., 1958) and so whether photochemical or diffusive equilibrium between $\mathrm{O}_{2}$ and 0 predominates, 0 will be the major constituent. Even a small amount of $\mathbb{N}_{2}$ dissociation can give rise to a large percentage of $\mathrm{N}$ at high heights due to diffusive separation. However, $\mathbb{N}$ will probably never be a predominate species until possible the lower exosphere. At sea level, the atmosphere is about $5.2 \times 10^{-4}$ percent helium, He. Thus about the same fraction of the atmosphere will be He at $h_{\mathrm{d}}(\approx 130 \mathrm{~km})$. Then assuming a diffusive equilibrium for He above $h_{d}$, it can be shown that He will be a minor atmospheric constituent throughout the F-region. According to Kuiper (1952), about $10^{9}$ atomic hydrogen, H, atoms are released per second per $\mathrm{cm}^{2}$ column due to photodissociation of water vapor near 80 $\mathrm{km}$. It must also escape at the top of the atmosphere at the same rate. Also, He escapes there at about $10^{5} / \mathrm{cm}^{2}-\mathrm{sec}$ (Mitra, 1952). Since H escapes about $10^{8}$ times more rapidly than He (due to its lighter mass), its concentration at the exosphere base must be about $10^{-4}$ that of He. Since He has already been argued to be a minor constituent, $\mathrm{H}$ must also be only a trace constituent in the F-region.

$$
\text { b. } I_{\infty}(\lambda)
$$

The radiations which may interact with the major constituents as postulated in the preceding paragraph are now examined. The atmosphere 
is known to be opaque to wavelengths from approximately 0-2900 A and from about 24 microns to $1 \mathrm{~cm}$. The second wavelength range does not carry enough energy to be an appreciable energy source and, as will be seen in the next section, has a small probability of being absorbed in the F-region. The wavelengths in the 24 micron to $1 \mathrm{~cm}$ range are thought to be emitted from the upper photosphere, and should be approximately equivalent to the radiation from a $6000^{\circ} \mathrm{K}$ black body. Since the peak energy density for a $6000^{\circ} \mathrm{K}$ distribution occurs at $4900 \mathrm{~A}$ and falls off approximately as $\lambda^{-4}$, the wavelength range starting at 24 microns will have little energy in it. The wavelengths between 2100-2900 A are almost exclusively absorbed by $\mathrm{O}_{3}$, so only the spectrum between 0 and 2100 $A$ is of interest. Farther into the ultraviolet the equivalent black bocy temperature of the sun becomes less, so that to agree with spectrographic (Johnson, et al., 1951) and photoelectric measurements (Friedman, 1955) a temperature of about $4500^{\circ} \mathrm{K}$ is taken for the range of wavelengths from 2100-1340 A. From 1350-1040 A occur a number of emission lines and particularly H Lyman $\alpha$ at $1216 \mathrm{~A}$. H Lyman $\alpha$ is thought to account for about 95 percent of the intensity in this wavelength range. Estimates of the incident intensity in this range have been made from rocket observations by several people and have been tabulated (Friedman, 1960a). In the range from 1040-60 A the fluxes have been measured at 210 and 255 $\mathrm{km}$ (Hinteregger, 1960) using photon counters. An extrapolation of these measurements to outside the atmosphere has been done (Denison, 1961) and these are taken to be representative of the solar flux in this range. Friedman (1960b) has tabulated the experimental measurements of hard $\mathrm{x}-$ rays in the regions $0-20 \mathrm{~A}$ and $44-60 \mathrm{~A}$. These will be taken as representative of the 0-60 A range and the amount of energy in 20-44 A range is obtained by interpolation. (All this information is shown in figure 3.) A smooth curve is drawn and the important emission lines, with an estimate as to their contained energy, is indicated. This will be taken as the rough spectrum of $I_{\infty}(\lambda)$ to be used for qualitative arguments and approximate quantitative comparisons. The graph refers mainly to sunspot maximum, since most of the measurements were taken then.

$$
\text { c. } A_{i}(\lambda)
$$

The point at IOA in figure 3 represents the total $x$-ray flux from 0-20 A. Therefore, pair production as an attenuating mechanism may evidently be neglected. The question of the loss of protons by Compton scattering will now be considered. As previously noted, an appreciable photon energy flux only exists for wavelengths above about $30 \mathrm{~A}$. With regard to the Compton effect this is a classical energy. The well known Klein-Nishina formula for the Compton scattering cross section per electron gives high cross sections for these energies (from about $10^{-16}-10^{-20}$ $\mathrm{cm}^{2}$ ). However, for such relatively low photon energies, the photon is hardly degraded at all in a scattering process. Also, the scattering 


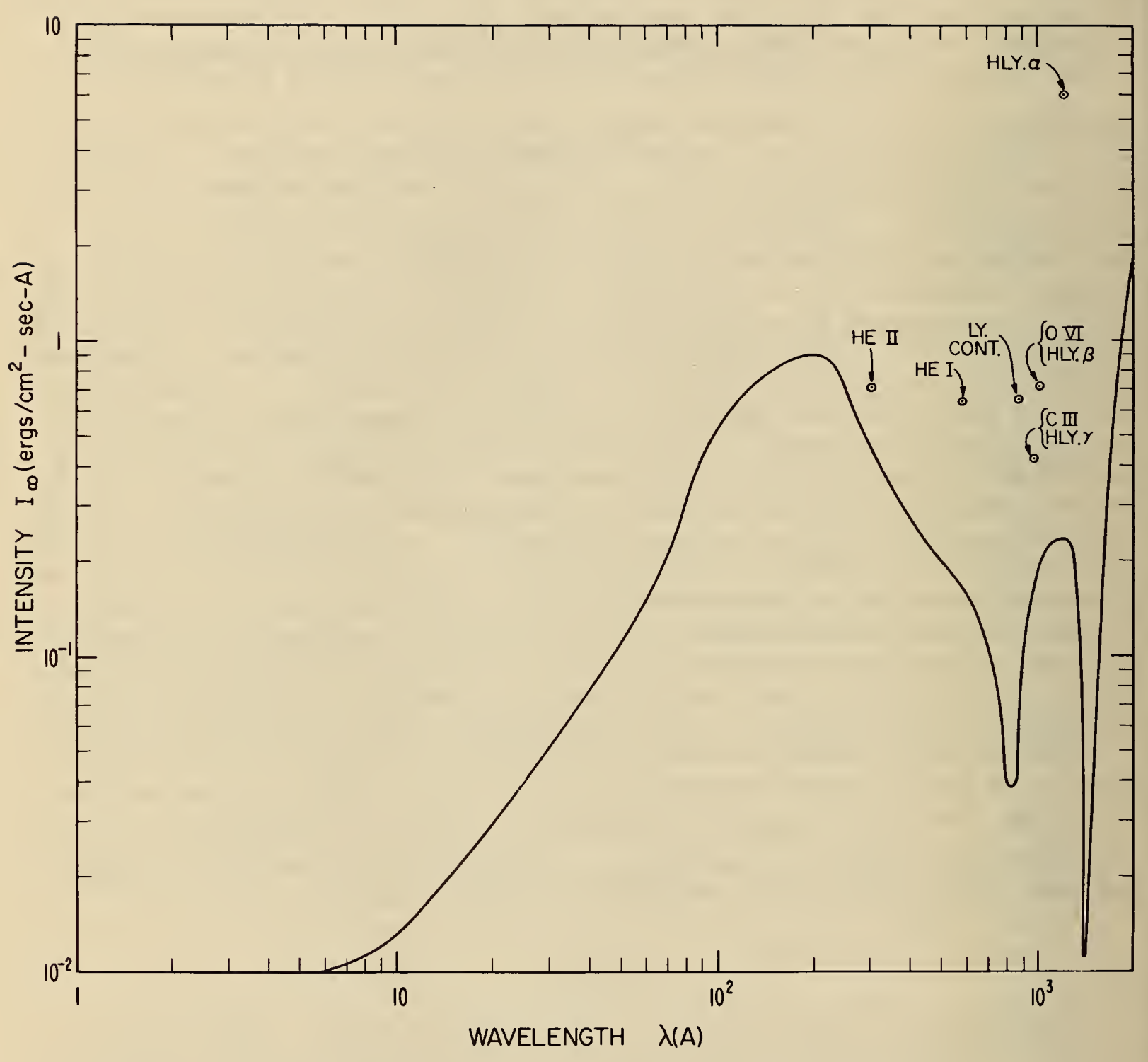

Figure 3

Solar Electromagnetic Flux Outside the Earth's Atmosphere 
angle is essentially $0^{\circ}$ in such low energy interactions. Thus, most of the photons in question will suffer negligible Compton scattering and this may be neglected as an attenuation mechanism. The remaining important attenuation processes are the following:

$\begin{array}{ll}\text { 1. Ionization } & \text { 4. Resonance absorption } \\ \text { 2. Dissociation } & \text { 5. Preionization } \\ \text { 3. Dissociative ionization } & \text { 6. Predissociation }\end{array}$

The absorption due to the three important F-region constituents will be discussed separately. In the case of $\mathrm{N}_{2}$ and $\mathrm{O}_{2}$, experimental measurements are possible, whereas theoretical estimates are virtually impossible. Thus experimental results will be exclusively used. In the case of 0 the situation is reversed and so theory must be used.

\section{(1) $\mathrm{A}_{\mathrm{O}_{2}}(\lambda)$ : Friedman (1960c) summarizes the recent work which has}

been done on the absorption cross section of the atmospheric constituents. For $\mathrm{O}_{2}$, starting at $2026 \mathrm{~A}$, there are absorption bands with a small cross section of about $10^{-23} \mathrm{~cm}^{2}$. At $1759 \mathrm{~A}$ absorption starts in the dissociation continuum with a cross section of about $5 \times 10^{-19} \mathrm{~cm}^{2}$. The dissociation continues until the first ionization potential of $\mathrm{O}_{2}$ at $1027 \mathrm{~A}$ where it becomes small compared to ionization. The absorption cross section reaches a broad, flat peak of about $1.6 \times 10^{-17} \mathrm{~cm}^{2}$ at $1450 \mathrm{~A}$. After this, absorption in bands is observed up to the ionization limit. It is especially to be noted that $H$ Lyman $\alpha$ has a low cross section of about $8.5 \times 10^{-21} \mathrm{~cm}^{2}$. From 1027-850 A an absorption continuum of superimposed bands is observed with a maximum cross section of about $4 \times 10^{-18}$ $\mathrm{cm}^{2}$. From 850-740 A, the continuous absorption has resonance bands superimposed with the absorption cross section reaching a maximum of $2.2 \mathrm{x}$ $10^{-17} \mathrm{~cm}^{2}$ at $510 \mathrm{~A}$. Most of the band absorption cross sections do not exceed $4 \times 10^{-17} \mathrm{~cm}^{2}$. Below $200 \mathrm{~A}$ the cross section is taken to be twice that of $O$ because of the lack of experimental data in this region and because this is a good approximation for high energy photons. A rough graph of $\sigma_{\mathrm{O}_{2}}(\lambda)=\mathrm{A}_{2}(\lambda) \mathrm{M}_{2} / \mathrm{N}_{\mathrm{O}}$ is shown in figure 4 .

(2) $\mathrm{A}_{\mathrm{NV}_{2}}$ : Above $1220 \mathrm{~A}, \mathrm{~N}_{2}$ is virtually transparent to electromagnetic radiation. Between 1200-796 A band absorption is present. Between the bands from 1040-910 A the absorption cross section is less than $4 \mathrm{x}$ $10^{-20} \mathrm{~cm}^{2}$, which may be taken as roughly representative of the cross section from 1200-796 A. At $796 \mathrm{~A}$ and $661 \mathrm{~A}$ there exist two ionization limits for $\mathrm{N}_{2}$. This accounts for the absorption continuum below $800 \mathrm{~A}$. 
Again, below $200 \mathrm{~A}$ the absorption cross section is taken as twice that for photoionization of $\mathrm{N}$. The absorption cross section for $\mathrm{N}_{2}$ below $800 \mathrm{~A}$ is shown in figure 5 where $\sigma_{\mathrm{N}_{2}}=\mathrm{A}_{\mathrm{N}_{2}} \mathrm{M}_{2} / \mathrm{N}_{0}$.

(3) $\mathrm{A}_{0}(\lambda)$ : As mentioned previously, only theoretical estimates are available as to the absorption cross sections for 0 . These are for absorption by photoionization and take no account of possible preionization. Since preionization is thought to occur in $\mathrm{O}_{2}$ from 950-850 A it would seem possible that some might occur in 0 near this wavelength range.

The values of $\sigma_{0}=A_{0} M_{O} / N_{0}$ are taken from the work of Bates and Seaton (1949) above $460 \mathrm{~A}$, Dalgarno and Parkinson (1960) from 460-22.5 $\mathrm{A}$, and Victoreen (1949) below $22.5 \mathrm{~A}$. The photoionization cross section is shown plotted in figure 6 . The various ionization limits appear as discontinuites on the cross section curve.

These cross section graphs are approximate and do not include all of the details of the cross sections in the wavelength range presented. A factor of error of about \pm 1.5 to \pm 2.0 is attached to all numbers quoted.

(4) $\epsilon(\lambda, h)$ : The thermal efficiency will vary with height through the different relative compositions of both the neutral and ionized parts of the atmosphere at different heights. It may vary with $\lambda$ through the different amounts of thermal energy which may be transferred to the neutral gas by photons of different energies. The variation of $\epsilon$ is a radiative transfer problem of some complexity and is avoided in the work of this paper by considering only the heat flux, i.e., the product $I_{\infty}(\lambda) \epsilon(\lambda, h)$.

The value of $\epsilon$ may be roughly estimated by considering that the main result of photonic interaction with the atmospheric F-region is photoionization and inquiring how these fast photoelectrons lose their energy. This has been done by Hanson and Johnson (1961) and the following discussion will be based on their work.

The energetic photoelectrons have several interactions. They may collide elastically with neutral particles, electrons, or positive ions, thus sharing their kinetic energy. They may also make inelastic collisions with neutral particles or positive ions leading to dissociation, ionization, or excitation. If the excitation energy is lost by radiation which is not locally absorbed, some of the prospective thermal energy is lost. Also, if the dissociated or ionized particles do not recombine locally, this internal energy is lost. The excitation energy may also be lost by collisional deactivation, in which case it may still 


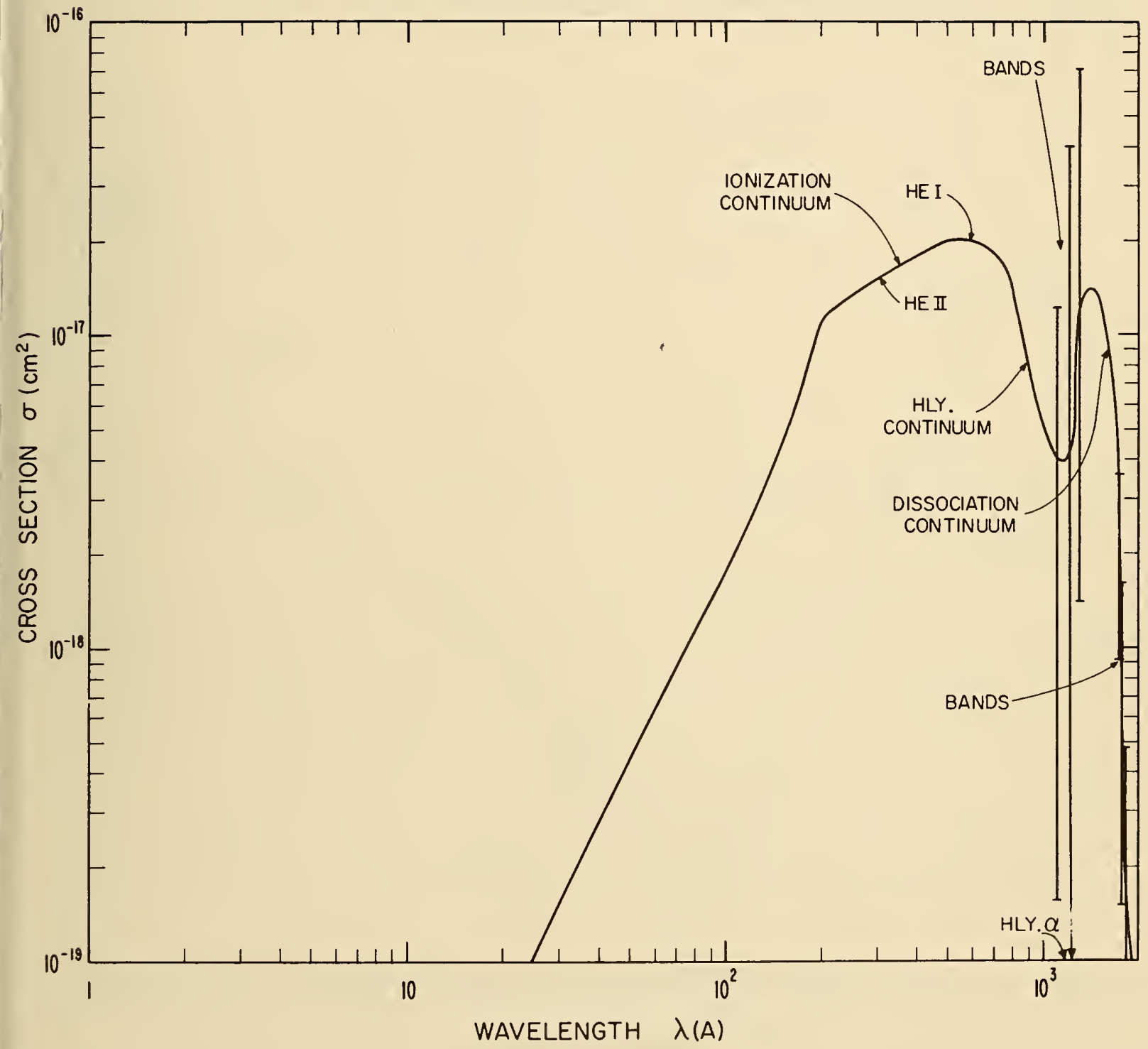

Figure 4

Absorption Cross Section of $\mathrm{O}_{2}$ Versus Wavelength 


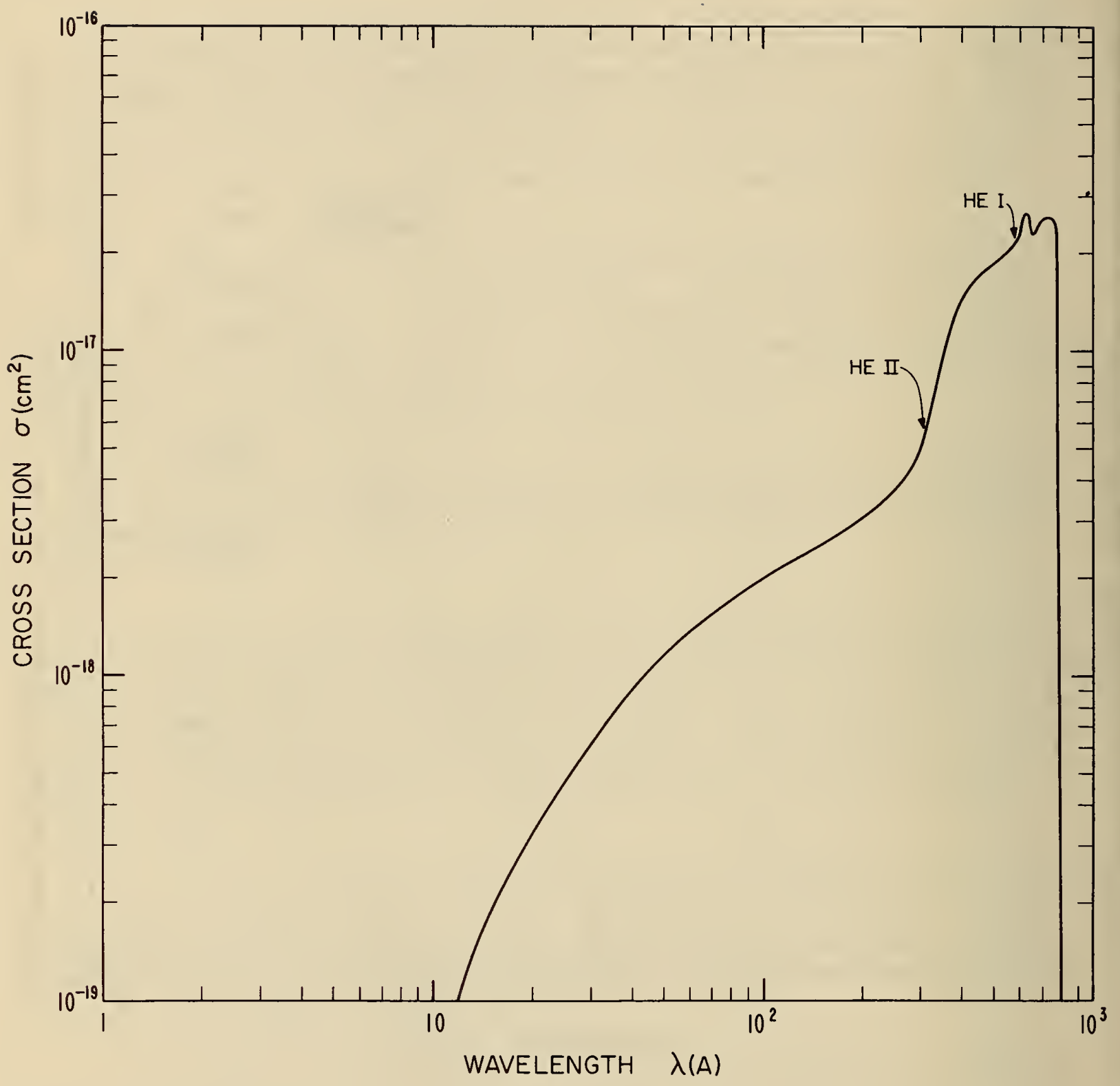

Figure 5

Absorption Cross Section of $\mathrm{N}_{2}$ Versus Wavelength 


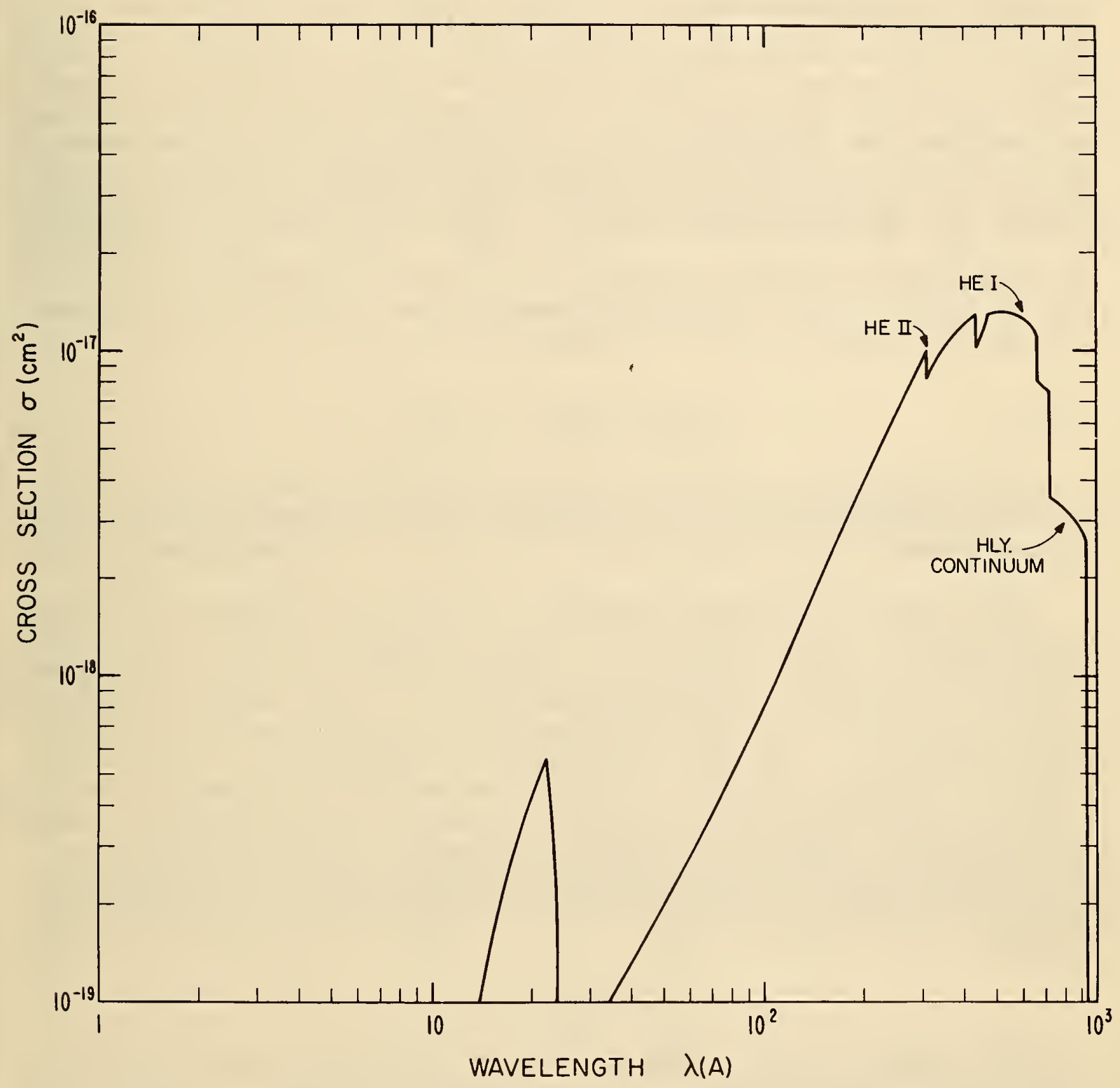

Figure 6

Photoionization Cross Section of 0 Versus Wavelength 
be available for atmospheric heating. Dissociative recombination may also be a heat source. Hanson and Johnson show that for most dissociative recombinations the maximum amount of fragmentation energy available is about $6 \mathrm{ev} /$ dissociation for $\mathrm{N}_{2}{ }^{+}$and $\mathrm{O}_{2}{ }^{+}$and about $2.7 \mathrm{ev} /$ dissociation for $\mathrm{NO}^{+}$(fragmentation energy is the kinetic energy of the two formed atoms). All the rest of the energy will be lost as radiation or unrecovered internal energy. Hanson and Johnson favor this heating mechanism and thus for $304 \mathrm{~A}$ photons get an $\epsilon$ of about 0.15. On the basis of this argument, about the same energy per photon would be available for any photon energetic enough to photoionize. Thus for purposes of argument it will be assumed that $\epsilon(h, \lambda)=\epsilon\left(h_{m}, \lambda\right)=6 /[$ h́c $/ \lambda]($ ev $)$, where $h_{m}$ is the height of maximum heat gain, $h$ ' is Planck's constant, and $c$ is the velocity of light. This then makes the heating effect proportional to the photon flux and not the energy flux.

\subsection{The Conduction Term}

This term may be written $1 / r^{2} \partial / \partial r\left[r^{2} \lambda(T) \partial T / \partial r\right]$ where $\lambda(T)$ is the thermal conductivity and $r$ is the distance from the Earth's center. If a plane geometry were considered the term would be $\partial / \partial \mathrm{h}[\lambda(\mathrm{T}) \partial \mathrm{T} / \partial \mathrm{h}]$.

The value of $\lambda(T)$ will be taken to consist of two parts, the first due to kinetic energy transfer in short range interactions (neutral gas conductivity) and the second due to kinetic energy transfer in long range Coulombic interactions (plasma conductivity). Since these conductivities will be seen to be very weak functions of the neutral particle and the plasma densities, they will be assumed to be independent of each other and their effects additive. As with cross section calculations, the theoretical estimates are best for atomic species where few empirical values are available. Conversely, experimental values for simple molecular gases are available while theoretical estimates are not as reliable. The conductivity for neutral and ionized gases will be considered separately.

\section{a. Neutral Gases}

(1) Simple gases: Using a method of successive approximations, Chapman and Cowling (1952b) solved the Boltzmann equation for the velocity distribution function. The first approximation is taken to be the Maxwellian distribution. Then, using the definition of thermal flux, the second approximation to $\lambda(T)$ is calculated in terms of the particle interaction. In the case of the spherically symmetric, rigid, elastic particles the second approximation is given as: 


$$
\lambda(T)=\frac{75}{64 \mathrm{~d}^{2}} \frac{\mathrm{k}^{3 / 2}}{(\pi \mathrm{m})^{1 / 2}} T^{1 / 2}
$$

Here $\mathrm{m}$ is the particle mass and $\mathrm{d}$ is the particle diameter. Chapman and Cowling (1952c) show that on this model the second approximation is almost exact.

Keyes (1952) has measured the thermal conductivity of several gases in the temperature range $73-1273^{\circ} \mathrm{K}$. All of the atoms, and the molecules without too much structure, follow the $\mathrm{T}^{1 / 2}$ law very closely. The conductivity for $\mathrm{N}_{2}$ and $\mathrm{O}_{2}$ as given by Keyes are used here and are (in ergs/ cm-sec- $\left.{ }^{\circ} \mathrm{K}\right)$ :

$$
\begin{aligned}
& \lambda_{\mathrm{N}_{2}}(\mathrm{~T})=191 \mathrm{~T}^{\mathrm{I} / 2} \pm 4 \% \\
& \lambda_{\mathrm{O}_{2}}(\mathrm{~T})=204 \mathrm{~T}^{\mathrm{I} / 2} \pm 4 \% .
\end{aligned}
$$

The two atoms measured by Keyes are He and A. Their conductivities (in ergs $/ \mathrm{cm}-\mathrm{sec}-{ }^{\circ} \mathrm{K}$ ) are:

$$
\begin{aligned}
& \lambda_{\mathrm{He}}(\mathrm{T})=530 \mathrm{~T}^{1 / 2} \pm 2 \% \\
& \lambda_{\mathrm{A}}(\mathrm{T})=75.5 \mathrm{~T}^{1 / 2} \pm 6 \% .
\end{aligned}
$$

If an interpolation between the conductivities for these two atoms is made, assuming that the $\left(\mathrm{d}^{2} \mathrm{~m}\right)^{-1 / 2}$ dependence shown in (3-11) is true and using values of d given by Brokaw (1961), a value of 361 is found for the coefficient of $\lambda_{0}(T)$ for atomic oxygen. Thus the conductivity of 0 is taken:

$$
\lambda_{0}(T)=361 T^{1 / 2} \pm 10 \%
$$

(2) Mixed gases: The problem of computing the conductivity in this case is complicated. Approximate formulas for the conductivities of mixtures are given, for example, by Chapman and Cowling (1952d) and by Brokaw (1961). Chapman and Cowling give the formula for the first approximation to the conductivity of a binary mixture. Then with the parameters appropriate to a gas composed of rigid, elastic, spherically symmetric molecules, the conductivity assumes the form: 


$$
\lambda_{1,2}(T)=\frac{\left(n_{1}+k_{1} n_{2}\right)\left(n_{1}+k_{2} n_{2}\right)}{\left(n_{1}+k_{3} n_{2}\right)\left(n_{1}+k_{4} n_{2}\right)} k_{5} T^{1 / 2} .
$$

Here $k_{i}(i=1-5)$ are rather complex functions of the molecular weights and conductivity coefficients of the gases 1 and 2 involved. The magnitudes of the $k_{i}$ are such that formula (3-13) may be approximately written:

$$
\lambda_{1,2}(T) \doteq \frac{k n_{1}+k^{\prime} n_{2}}{n_{1}+n_{2}} T^{I / 2} \text {. }
$$

Here $k$ and $k^{\prime}$ are new constants which may be identified with the simple gas conductivities. This is equivalent to weighting the simple gas conductivities by their number densities. Keyes (1952) also gives the conductivity of a mixture of $\mathrm{N}_{2}$ and $\mathrm{CO}_{2}$ as a function of mole percent $\mathrm{N}_{2}$ at temperatures $323-623^{\circ} \mathrm{K}$. Although $\mathrm{CO}_{2}$ does not follow a $\mathrm{T}^{\mathrm{I} / 2}$ law too closely, it is still seen that a weighting of the simple gas conductivities by the number densities gives a good approximation to the mixture conductivity. At $520^{\circ} \mathrm{K}$, for example, a mixture of fifty percent $\mathbb{N}_{2}$ and fifty percent $\mathrm{CO}_{2}$ gives a conductivity exactly halfway between the conductivities of pure $\mathrm{N}_{2}$ and $\mathrm{CO}_{2}$, just as (3-13-1) predicts. Thus the defining equation for the mixture conductivity is taken to be:

$$
\begin{aligned}
\lambda_{m}(T) & =\sum_{i} n_{i} \lambda_{i}(T) / \sum_{i} n_{i} \cdot \\
& \text { b. Plasma }
\end{aligned}
$$

In general, the ability of a gas to conduct heat is directly proportional to the value of the mean free path of a typical particle in the gas. To see this, consider a plane in the gas across which thermal energy is being transported by the molecules on one side of the plane to the molecules on the other side. The longer the mean free path of the molecules on one side of the plane, the greater the chance that these molecules will be able to cross this plane and deliver their kinetic energy to the molecules on the other side. A general kinetic theory result is that the mean free path of the particle in a gas is inversely proportional to the interaction cross section. Thus the thermal conductivity is inversely proportional to the interaction cross section. In neutral gases the interaction cross section is relatively small and of the order of the physical cross sectional area of the particles. In ionized gases however, where long range Coulomb forces are present, the interaction cross section will be quite large. Therefore, the thermal 
conductivity of a plasma is usually quite small. Chapman and Cowling (1952e) give the classical (at higher temperatures quantum statistics are unnecessary) expression for the thermal conductivity of a simple gas of particles having a Coulomb interaction. Here $\lambda(T)$ is proportional to $\mathrm{T}^{5 / 2} \mathrm{~m}^{1 / 2} / \mathrm{q}^{4} \log _{10} \dot{n}$, where $\mathrm{q}$ is the particle charge. Calculations for a $1000^{\circ} \mathrm{K}$ electron gas show the conductivity to be about $1 \mathrm{erg} / \mathrm{cm}-\mathrm{sec}-{ }^{\circ} \mathrm{K}$, compared with a pure 0 neutral particle conductivity of about $10^{4}$ ergs/ $\mathrm{cm}-\mathrm{sec}-{ }^{\circ} \mathrm{K}$. Only if the electron temperature is about $10,000^{\circ} \mathrm{K}$ will the conductivity be comparable to that of the neutral gas. Hanson and Johnson (1961) estimate an electron temperature maximum of about $1600^{\circ} \mathrm{K}$ in the F-region, but point out their estimate may be low because of an overestimate of the excitation cross section of 0 . A $10,000^{\circ} \mathrm{K}$ temperature implies an average electron energy of about $6.5 \mathrm{e} \cdot \mathrm{v}$. Then considering the thermalizing effect of a $1000^{\circ} \mathrm{K}$ electron gas on these fast electrons, even in the vicinity of $200 \mathrm{~km}$ where the rast electron production is greatest and the $1000^{\circ} \mathrm{K}$ electron gas density quite small, shows it will take only about $0.1 \mathrm{sec}$. for thermalization.

The conduction of a heavy ion gas will be a factor of about 40 greater than that of an electron gas at the same temperature, but the ions will undoubtedly have almost the same temperature as the neutral gas; thus their contribution can be neglected. On the basis of the previous arguments (and that at temperate latitudes, the Earth's magnetic field impedes vertical conduction) the ionized particle conductivity is neglected.

\section{c. Turbulent Conduction}

If turbulence is present in the atmosphere, the thermal conduction will be much greater because of the increased chance for energy transferring collisions between the molecules. As previously mentioned, diffusive equilibrium is quite likely to prevail above about $120-130 \mathrm{~km}$, rendering doubtful any pronounced turbulence (which would lead to mixing) above these heights. Also, Johnson (1958) has argued against the existence of turbulence which was postulated by Booker (1956) as being present as high as $170 \mathrm{~km}$. This turbulent level is suspected since the radio star scintillation level is a function of the inhomogeneities in the electron-ion gas, and these inhomogeneities were taken to be too large (according to Johnson) at $170 \mathrm{~km}$ by Booker. Thus it will be assumed here that above $130 \mathrm{~km}$ turbulent conduction may be neglected.

\subsection{The Loss Term, I ( h, t )}

Bates (1951) has discussed the radiative cooling of the atmosphere in some detail on the basis of transition probabilities and constituent 
abundance. He concluded that the only significant radiative loss should be from the ${ }^{3} \mathrm{P}_{1}-{ }^{3} \mathrm{P}_{2}$ transition in the atomic oxygen ground state. The energy difference is $0.020 \mathrm{e} \cdot \mathrm{v}$. and therefore is of the order of F-region kinetic temperatures. Thus the upper level may be populated and depopulated by thermal energy collisions giving rise to net radiative loss (if the photons are not reabsorbed). Bates assumes and shows that the distribution of atoms between the two levels is probably characteristic of the gas temperature, T, i.e., that thermal excitation of the upper level is much faster than photon emission. Since the radiative temperature of this transition is considerably less than the F-region kinetic temperature, $T$, it can be assumed that all the emitted photons escape the F-region. On this basis, Bates gives the following expression for this radiative loss:

$$
\begin{aligned}
L(h, t)= & 1.67 \times 10^{-18} \cdot n_{0}(h) \exp (-228 / T) /[1+0.6 \exp (-228 / T) \\
& +0.2 \exp (325.3 / T)] \operatorname{ergs} / \mathrm{cm}^{3}-\mathrm{sec} .
\end{aligned}
$$

This is assumed to be the entire loss term, $L(h, t)$.

\subsection{The Heat Capacity Term}

In the solution of the equation with this term included (the nonsteady state) the density and the specific heat change with both height and time. It is to be noted, however, that $\rho$ and $c_{v}$ have no explicit time dependence, but only an implicit dependence through the temperature. This implicit time dependence is treated in the solution of the nonsteady state equation by assuming an equilibrium is attained around each separate time the equation is solved. The specific heat of a mixture of perfect gases is given, by analogy with $(1-3)$ to $(1-5)$ as the sum of the specific heats of the individual gases weighted by the mass densities of the individual gases. Thus $c_{v}$ is given:

$$
c_{v}=\sum_{i} \rho_{i} c_{v_{i}} / \sum_{i} \rho_{i} \cdot
$$

Here $c_{v_{i}}$ is the specific heat of the $i^{\text {th }}$ constituent. Thus the heat balance equation (2-10-2) is now written: 


$$
\begin{gathered}
\left(\sum_{i} \rho_{i} c_{v_{i}} / \sum_{i} \rho_{i}\right) \cdot \rho(h, t) \partial T(h, t) / \partial t \\
=\frac{1}{(a+h)^{2}} \partial / \partial h\left\{(a+h)^{2} \sum_{i} n_{i} \lambda_{i} / \sum_{i} n_{i}[\partial T(h, t) / \partial h]\right\} \\
+\int_{\begin{array}{c}
\text { ionizing } \\
\text { wavelengths }
\end{array}} \epsilon(\lambda, h) I_{\infty}(\lambda) \sum_{i} A_{i}(\lambda) \rho_{i}(h) \exp [-\tau(\lambda, h) \sec x] d \lambda \\
-1.67 \times 10^{-18} n_{0}(h) \exp (-228 / T) /[1+0.6 \exp (-228 / T) \\
+0.2 \exp (-325 \cdot 3 / T)] .
\end{gathered}
$$

where $a$ is the average radius of the earth.

\section{SOLUTIONS OF EQUATIONS}

\section{I Terms in Final Form of Heat Balance Equation}

In order to attempt a solution of (3-17), several further approximations must be made in accordance with the discussion of Chapter 3 . These assumptions will be discussed for each term in equation (3-17).

\section{a. Heat Capacity Term}

As previously indicated, this term is quite likely negligible ( $\approx$ ten percent of gain term) during most of the day and at most heights. Therefore the solution of (3.17) is attempted first for the quasi-steady state and second for the non-steady state.

\section{b. The Gain Term}

In order to integrate this term over $\lambda$, the integration is replaced by a summation. Wavelength ranges are selected and step functions are sbustituted for $A_{i}(\lambda)$ and $\epsilon(\lambda, h) I_{\infty}(\lambda)$. These ranges are selected according to the graphs of $I_{\infty}(\lambda)$ and $A_{i}(\lambda)$ shown in the previous chapter. For the non-steady state two wavelength ranges are selected, centered on the two He lines at $304 \mathrm{~A}$ and $584 \mathrm{~A}$. For the quasi-steady state an additional range centered in the $\mathrm{H}$ Lyman continuum around $900 \mathrm{~A}$ is used. The approximate ranges of the intervals are:

1. He II range: $0-475 \mathrm{~A}(200-475 \mathrm{~A})$

2. He I range: $475-720 \mathrm{~A}(475-720 \mathrm{~A})$

3. H Lyman continuum range: 720-1760 A (720-911 A). 
The numbers in parentheses are the actual approximate ranges considered to contribute to F-region heating. The flux quantity considered is always the product, $\in I_{\infty}(\lambda)$ (the heat flux), now considered only as a function of wavelength.

The atmospheric composition for the non-steady state is taken to be 0 and $N_{2}$ in a diffusive equilibrium above the selected $h_{d}$. For the quasisteady state $\mathrm{O}_{2}$ is also taken as a constituent and is assumed to be in diffusive equilibrium above the selected $h_{d}$ and to have a fixed degree of dissociation at $h_{d}$. No dissociation of $N_{2}$ is considered in accordance with the arguments of Chapter 3, first section. For large zenith angles $(x)$, the path length traveled by a solar electromagnetic wave is not simply given by dh secx (as in a plane atmosphere), but by $d h \operatorname{Ch}(x, R)$ where $\mathrm{Ch}(x, R)$ is called the Chapman function and $\mathrm{R} \equiv(a+h) / H(h)$. Here " $a$ " again is the Earth's radius. This function takes account of the curvature of the Earth's atmosphere. Up to $x=90^{\circ}, \mathrm{Ch}(x, R)$ is a weak function of height and so may be substituted directly into the gain term. Thus the gain term may be written:

$$
\left.G(h, t)=\sum_{j} \in I_{\infty}\right)_{j} \sum_{i} A_{i j} \rho_{i}(h, t) \exp [-\tau, j(h, t) \operatorname{Ch}(x, R)] . \quad(4-I)
$$

Here $\left.\in I_{\infty}\right)_{j}$ is the total heat flux in the $j^{\text {th }}$ wavelength range, $A_{j i}$ is the absorption cross section for the $i^{\text {th }}$ constituent in the $j^{\text {th }}$ wavelength range (taken from figures 4 to 6 ), and $\tau_{j}=\sum_{i} \int_{h}^{h}{ }^{h} A_{j i} \rho_{i}$ dh where
$h_{m}$ is some high F-region height.

\section{c. The Loss Term}

As seen in (3-15) the loss term is proportional to $\mathrm{n}_{0}$ and a function of $T$. Computation shows that the $T$ dependent coefficient varies from about 7.1 to 8.6 from temperatures of $400-1500^{\circ} \mathrm{K}$. Thus an average value of 8.0 is taken and the loss term is then:

$$
L(h, t)=9.57 \times 10^{-19} n_{0}(h, t)\left(\text { ergs } / \mathrm{cm}^{3}-\mathrm{sec}\right)
$$

\section{d. The Conduction Term}

In the integration of the heat balance equation the planar approximation is adopted in the sense that integrals of the form $(a+h)^{-2} \int$ 
$(a+h)^{2} f(a+h) d h$ are taken to be equal to $\int_{(a+h)}^{\infty} f(a+h) d h$. Then the conduction term maintains its form as in (3-17). The form of the heat balance equation which is solved is

$$
\begin{aligned}
& \left(\sum_{i} \rho_{i} c_{v_{i}} / \sum_{i} \rho_{i}\right) \rho(h, t) \partial T(h, t) / \partial t \\
& =\frac{1}{(a+h)^{2}} \partial / \partial h\left[(a+h)^{2}\left(\sum_{i} n_{i} \lambda_{i} / \sum_{i} n_{i}\right) \partial T(h, t) / \partial h\right] \\
& \left.+\sum_{j} \in I_{\infty}\right) \sum_{j} A_{j i} \rho_{i}(h, t) \exp [-\tau, j(h, t) c h(x, R)] \\
& -9.57 \times 10^{-19} n_{0}(h, t) . \\
& 4.2 \text { Boundary Conditions }
\end{aligned}
$$

The boundary conditions will be considered separately and the effect of the variation of each will be shown in 5 .

\section{a. The Height Range Considered}

The height range is taken as $130-500 \mathrm{~km}$. This includes most of the F-region. The upper boundary is taken such that the atmosphere is approximately isothermal above it. The lower boundary must be taken low enough to insure the absorption of most of the incident radiation and high enough to avoid complications due to E-layer absorption and turbulence. One-hundred thiry $\mathrm{km}$ is considered a good compromise.

b. The Height, $h_{d}$, and the Degree of $\mathrm{O}_{2}$ Dissociation

The detailed composition is considerably affected and the total density somewhat affected by the choice of this parameter. Since it is thought to lie between 110 and $150 \mathrm{~km}$, the choice of $130 \mathrm{~km}$ has been made. The degree of $\mathrm{O}_{2}$ dissociation is poorly known as a function of height. The $\mathrm{O}_{2}$ mass density and the total mass density are known moderately well from 100-200 km, but the degree of dissociation still depends on $h_{d}$. For the $h_{d}$ of $130 \mathrm{~km}$ chosen, a dissociation degree of 80 percent has been taken as yielding the best fit to the observed data. 
c. The Temperature and the Mass Densities at $130 \mathrm{~km}$

Height and time density profiles are observationally known in the F-region from satellite and rocket measurements. From these, scale heights may be derived knowing only a boundary pressure at some high height. This is done by integration of the hydrostatic equation from some high height to the (lower) height in question and use of the relation that $\mathrm{H}(\mathrm{h})=\mathrm{p}(\mathrm{h}) / \mathrm{p} /(\mathrm{h}) \mathrm{g}(\mathrm{h})$. The boundary pressure will affect the situation at low heights very little. Thus a scale height of $15 \mathrm{~km}$ is taken from Kallmann's (1960) data at $130 \mathrm{~km}$. This fixes the temperature since the choice of $h_{d}$ and the dissociation degree of $\mathrm{O}_{2}$ fixes the molecular weight. Thus a temperature of $450^{\circ} \mathrm{K}$ is taken at $130 \mathrm{~km}$ for the quasi-steady state and $420^{\circ} \mathrm{K}$ at $130 \mathrm{~km}$ for the non-steady state. Recent experimental work (Blamont, et al., 1961), using the twilight flash from rocket injected sodium, has measured the temperature at $130 \mathrm{~km}$ to be about $350^{\circ} \mathrm{K}$. This would suggest a lowering of $h_{d}$ and an increase in the $\mathrm{O}_{2}$ dissociation to maintain $\mathrm{H}(130)$ at its observed value. Boundary conditions consistent with the temperatures of Blamont, et al., are discussed in 5. Most of the density measurements have been made during sunspot mini mum, during the winter, or at high latitudes. Thus the value of $\rho(130)$ is taken to be $10^{-11} \mathrm{gm} / \mathrm{cm}^{3}$, which is near the upper limit of the measurements. This implies boundary densities of $\rho_{0}(130)=1.56 \times 10^{-12} \mathrm{gm} / \mathrm{cm}^{3}$, $\rho_{\mathrm{O}_{2}}(130)=7.73 \times 10^{-13} \mathrm{gm} / \mathrm{cm}^{3}$, and $\rho_{\mathrm{N}_{2}}(130)=7.67 \times 10^{-12} \mathrm{gm} / \mathrm{cm}^{3}$ for the quasi-steady state and $\rho_{\mathrm{N}_{2}}(130)=8.4 \times 10^{-12} \mathrm{gm} / \mathrm{cm}^{3}$ and $\rho_{0}=1.64$ $\times 10^{-12} \mathrm{gm} / \mathrm{cm}^{3}$ for the non-steady state case.

\section{d. The Conductive Flux at the Upper Boundary}

This is the so called coronal conduction term and includes any thermal energy conducted into the atmosphere from above $500 \mathrm{~km}$. Since the conductivity at these heights is large, a small temperature gradient of about $1^{\circ} \mathrm{K} / \mathrm{km}$, which is suggested by scale height data, will give a conductive flux of about $1 \mathrm{erg} / \mathrm{cm}^{2}-\mathrm{sec}$. It is felt, however, that scale height data at high heights is unreliable and an isothermal exosphere is more likely. Thus the coronal conduction flux $\mathrm{F}_{\mathrm{m}}(\mathrm{ergs} / \mathrm{sec})$, is taken to be zero.

\subsection{Solutions of the Heat Balance Equation}

\section{a. Quasi-steady State}

In this case, the problem is a non-linear, second order total differential equation. If the $\rho_{i}$ and $\tau_{j}$ are known as analytic functions of 
T, an analytic solution of (4-3) might be effected. In particular, if a single constituent with constant scale height is considered, the equation may be solved in terms of exponential integrals. However, in general, numerical methods must be used because of the non-linearity of the equation. The formal solution is:

$$
\begin{aligned}
{\left[T(h) / T\left(h_{0}\right)\right]^{3 / 2} } & =1+\frac{3}{2 T\left(h_{0}\right)^{3 / 2}} \int_{h_{0}}^{h} \frac{I}{\lambda(h)}\left\{\sum_{i} \in I_{\infty}\right)_{i}\left[1-\exp \left(-\operatorname{Ch}\{x, R\} \tau_{i}\{h\}\right)\right] \\
& \left.-\int_{h}^{h} 9.57 \times 10^{-19} n_{0}(h) d h+F_{m} /(a+h)^{2}\right\} d h \quad(4-4)
\end{aligned}
$$

Here $\lambda(h)=\sum n_{i} \lambda_{i} / \sum n_{i}, \lambda_{i}$ is the conductivity coefficient for the $i^{\text {th }}$ i i

constituent, and $h_{m}$ and $h_{0}$ are the upper and lower boundary heights.

The method of solution is to assume a temperature distribution, compute the $\rho_{i}$ 's using (1-10), and then compute a new temperature distribution using (4-4). The process is then iterated until the successive temperature and density distributions are colısistent. In practice, about two iterations are necessary to achieve convergence.

\section{b. Non-steady State Solution}

When the heat capacity term is included, the equation to be solved is $\approx$ non-linear, second order, partial differential equation in $h$ and $t$. For an analytical solution of such an equation, three boundary conditions on the dependent variable are necessary. For example, $T\left(h_{0}, t\right), T\left(h_{m}, t\right)$, and $T\left(h, t_{0}\right)$ (where $t_{0}$ is some initial time) may be specified. The standard method of analytical solution of conduction problems is according to a transform theory, usually using Iaplace transforms. This reduces the partial differential equation to a total differential equation which is then solved for the transform of the required function. Then if an inverse transform is tabulated or can be calculated, $T(h, t)$ is obtained. The non-steady state problem cannot, in general, be solved in closed form except for situations which are not physically attainable. A list of conduction geometries and boundary condition sets which may be solved is available (Carslaw and Jaeger, 1959). If, for example, we consider a two dimensionally infinite atmosphere with all of the suggested boundary conditions constant in height and time and with $\mathrm{c}_{\mathrm{v}} \rho(\mathrm{z}, \mathrm{t}) / \mathrm{At} \mathrm{t}^{1 / 2}$ being constant, then the solution of the "nighttime" problem, with only the heat capacity term and conductive loss, may be written: 


$$
\begin{gathered}
\left.T(h, t)=T\left(h, t_{0}\right)+L T\left(h_{0}, t\right)-T\left(h, t_{0}\right)\right] \\
{\left[1-2 / \pi^{I / 2} \int_{0}^{0\left(h_{0}-h\right) /\left(2 c_{V} p / T^{I / 2} A\right)^{I / 2} t} \exp \left(-t^{2}\right) d t\right] .}
\end{gathered}
$$

The method of solution attempted here is to use an iterative method based on the quasi-steady state calculations. The term $\int_{h}^{h} m_{\left\{-c_{v}\right.}(h) \rho(h, t)$ $\partial T(h, t) / \partial t\} d h$ is added to the integrand in (4-4). Using values of this term calculated from the quasi-steady state densities and temperatures, new densities and temperatures iteratively consistent in height are computed. The process is then iterated to obtain time as well as height convergence. An alternative method would be to integrate once over time and use the conduction term as the correction term.

\section{RESUITS AND DISCUSSION}

\subsection{Results}

\section{a. Quasi-steady State}

The first and one of the most important results is to determine the heat fluxes necessary to give a daytime mass density profile which agrees with observation. One of the most recent daytime mass density profiles is that of KalImann (1961); she has made a careful compilation of most of the current observational data. Thus Kallmann's mass density profiles (both for daytime and nighttime) are used and are shown by $x$ 's on figures $7 \mathrm{a}, 7 \mathrm{~b}, 7 \mathrm{c}$ and 10. Also, Kallmann's scale height profiles, derived from her density profiles, are taken as the observational scale heights and are shown as $\mathrm{X}^{\prime} \mathrm{s}$ in figures $8 \mathrm{a}, 8 \mathrm{~b}, 8 \mathrm{c}$ and 9.

In figures $7 \mathrm{a}, 7 \mathrm{~b}, 7 \mathrm{c}$ and $8 \mathrm{a}, 8 \mathrm{~b}, 8 \mathrm{c}$ are shown mass density versus height and scale height versus height curves. There curves are drawn assuming a heat flux sum of $1.1 \mathrm{ergs} / \mathrm{cm}^{2}-\mathrm{sec}$ (Hunt and VanZandt, 1961) and a zenith angle, $x$, of zero degrees. This $x$ is chosen in order to get maximum densities that may then be compared to the observational daytime densities which are maximum daytime densities. Each curve refers to different relative amounts of heat flux in the three wavelength ranges. The purpose of these curves is to: (1) see how sensitive the mass density and scale height curves are to changes in the relative amounts of heat fluxes in the three wavelength ranges and (2) to determine which heat flux set gives mass density and scale height curves of similar shape to the observational curves.

It is seen that a heat flux of $1.1 \mathrm{ergs} / \mathrm{cm}^{2}-\mathrm{sec}$ gives densities somewhat lower than the observational curves. This is because of Hunt 


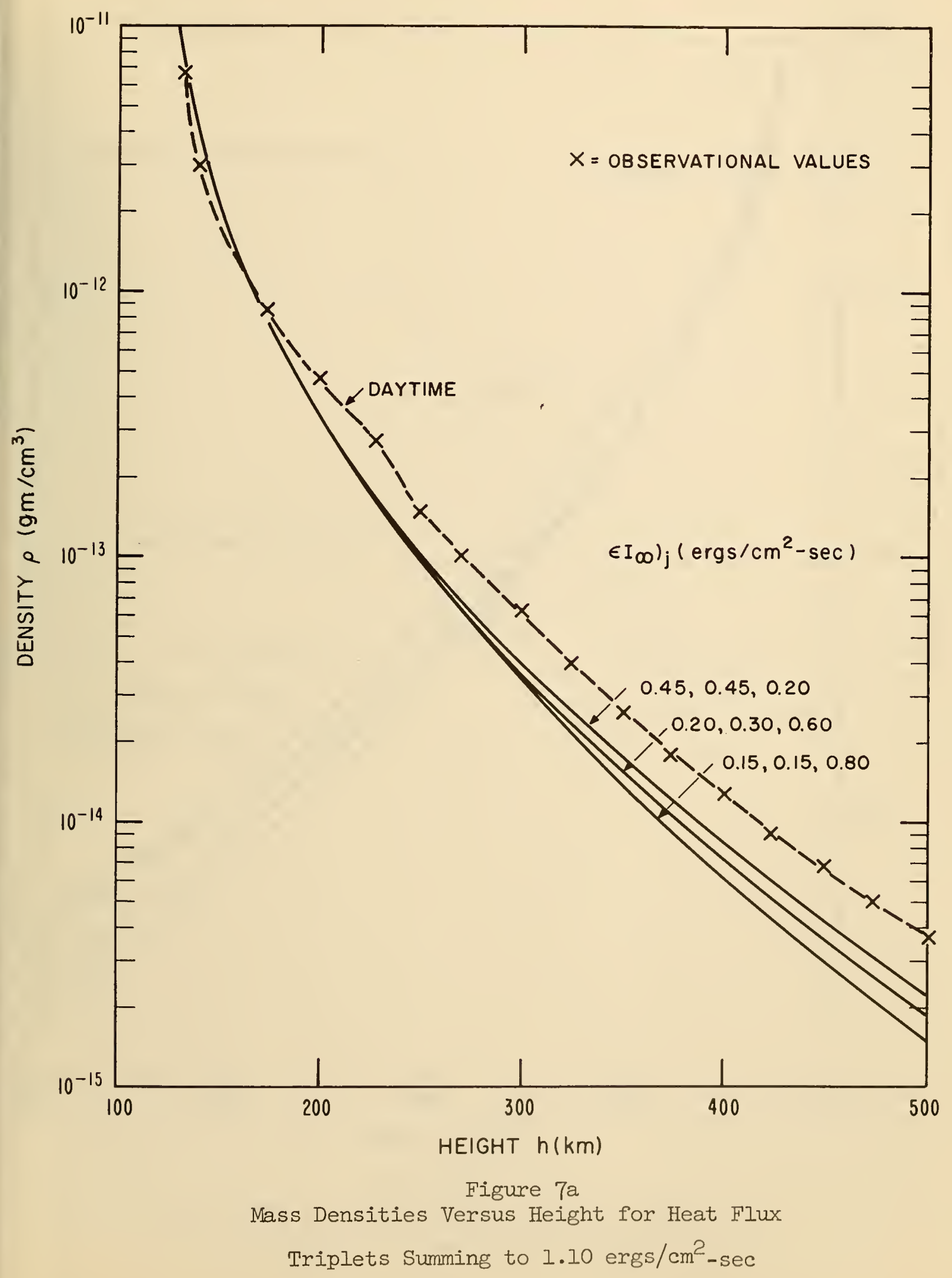




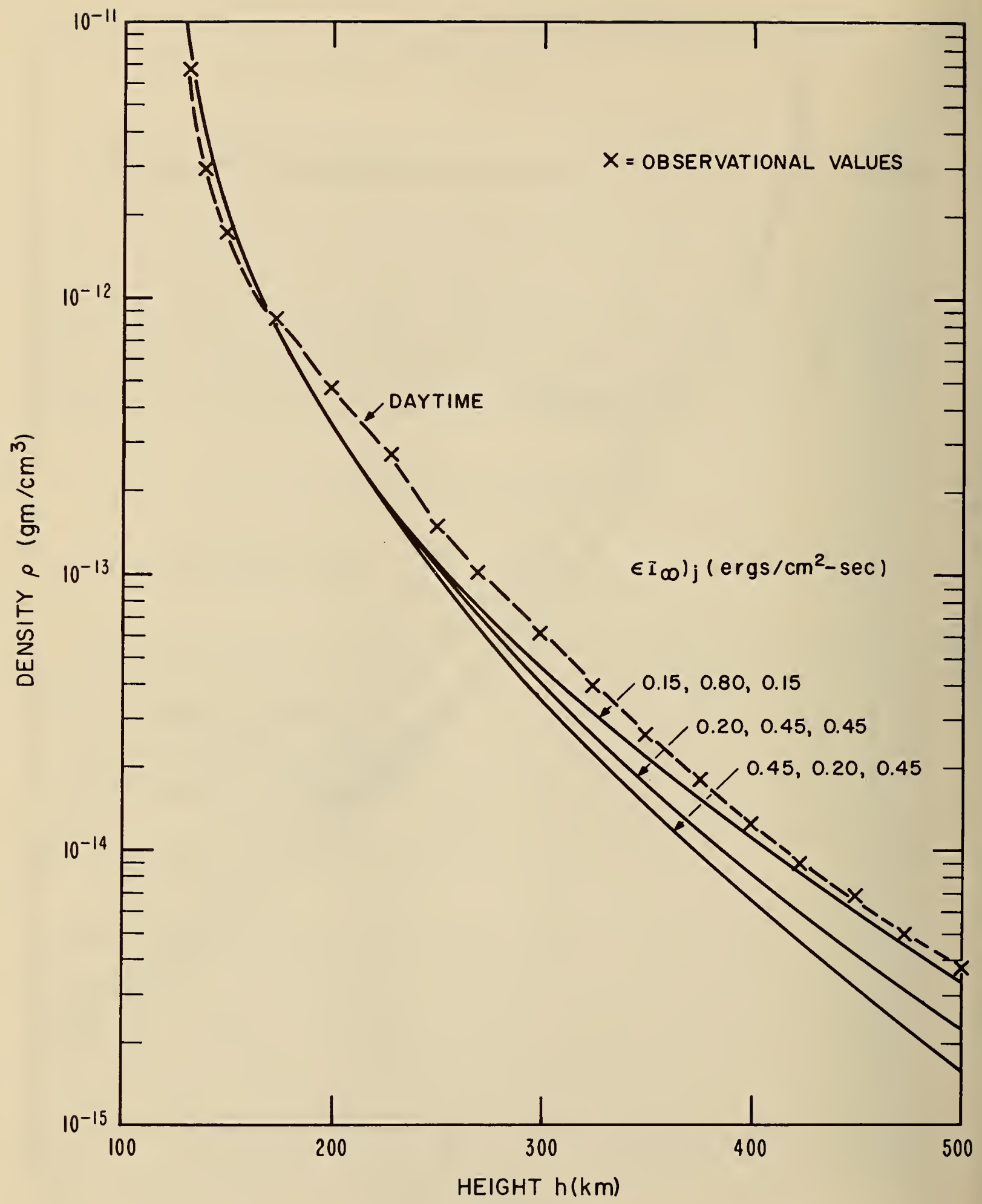

Figure $7 \mathrm{~b}$

Mass Densities Versus Height for Heat Flux

Triplets Summing to $1.10 \mathrm{ergs} / \mathrm{cm}^{2}-\mathrm{sec}$ 


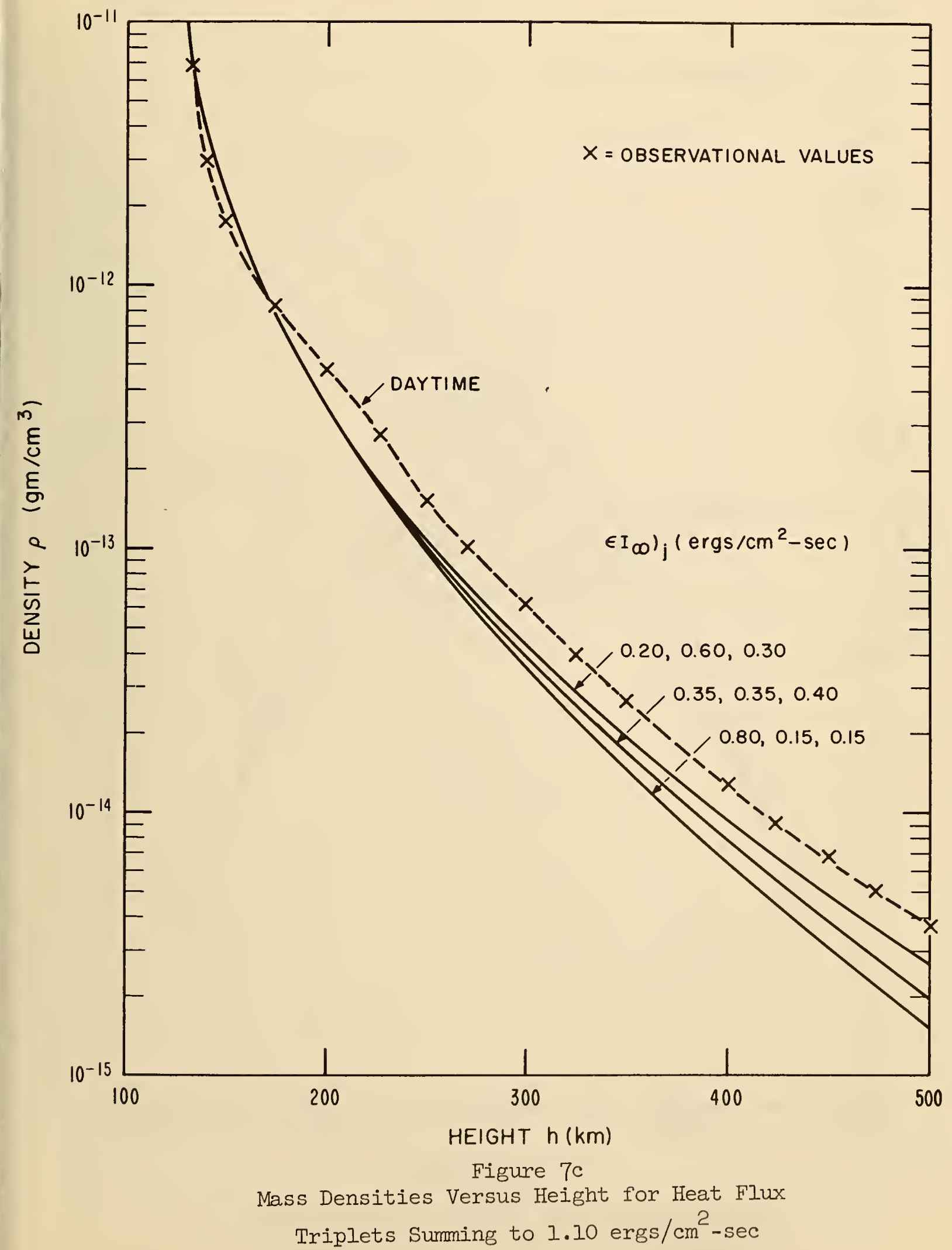




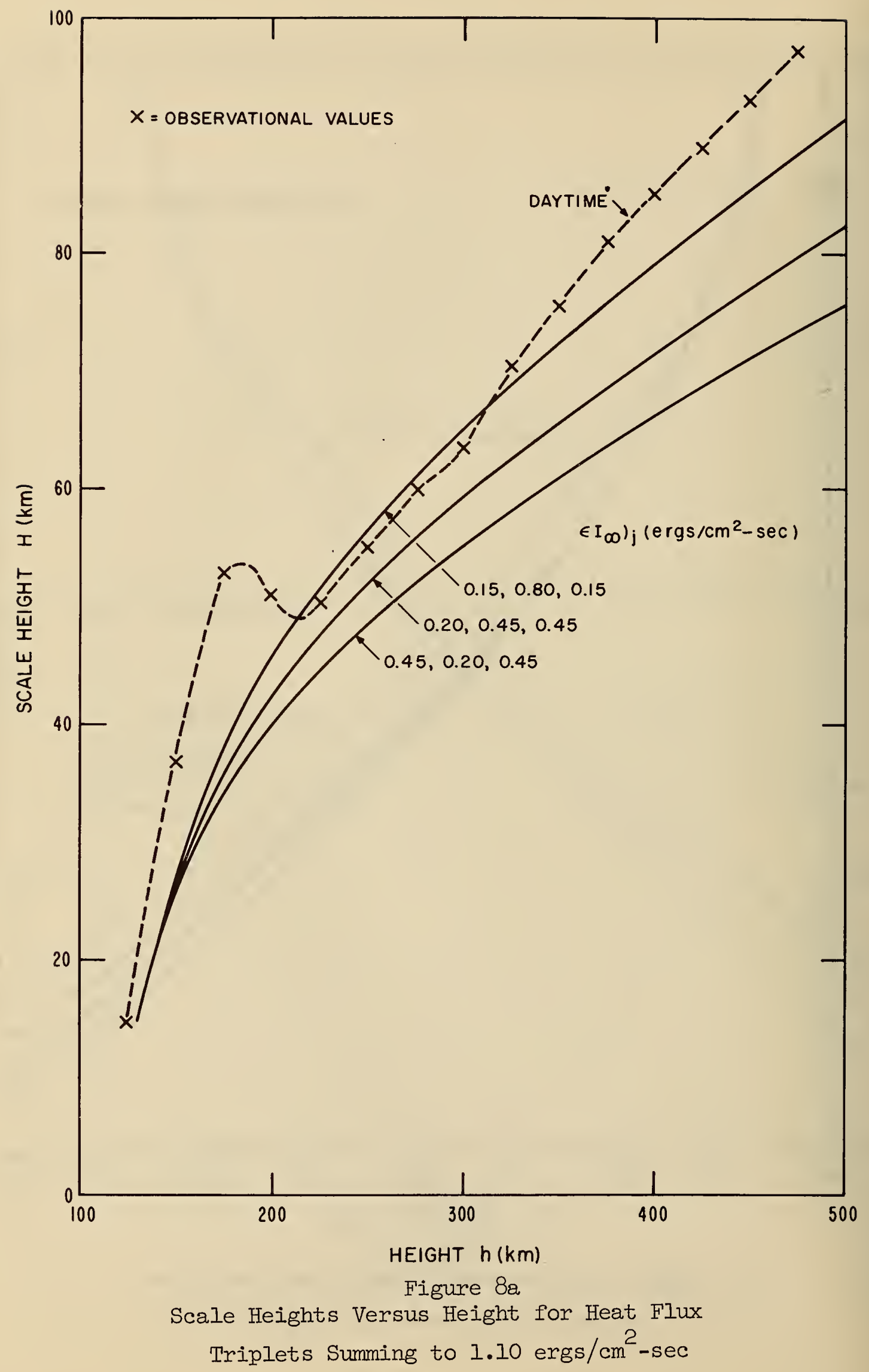




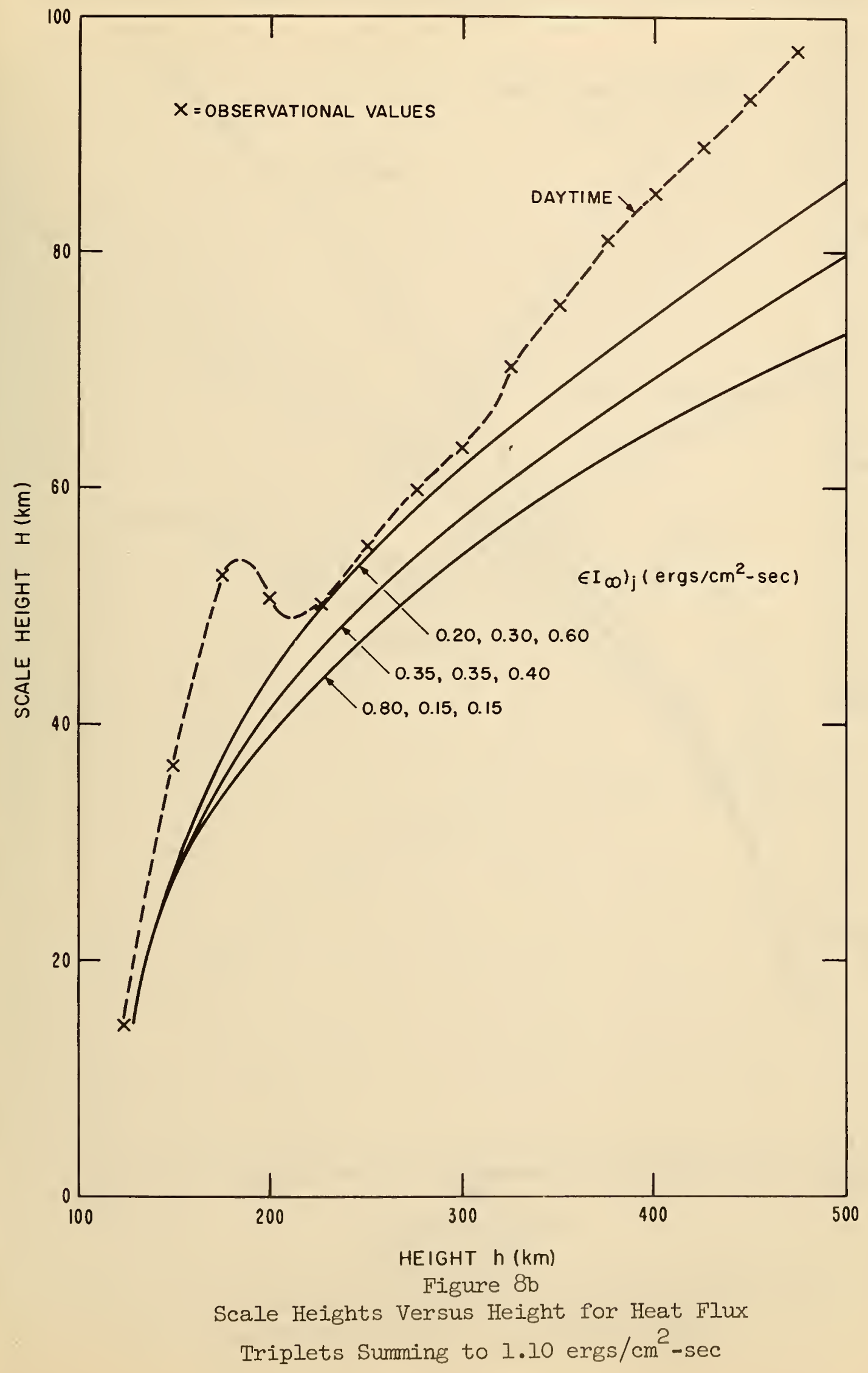




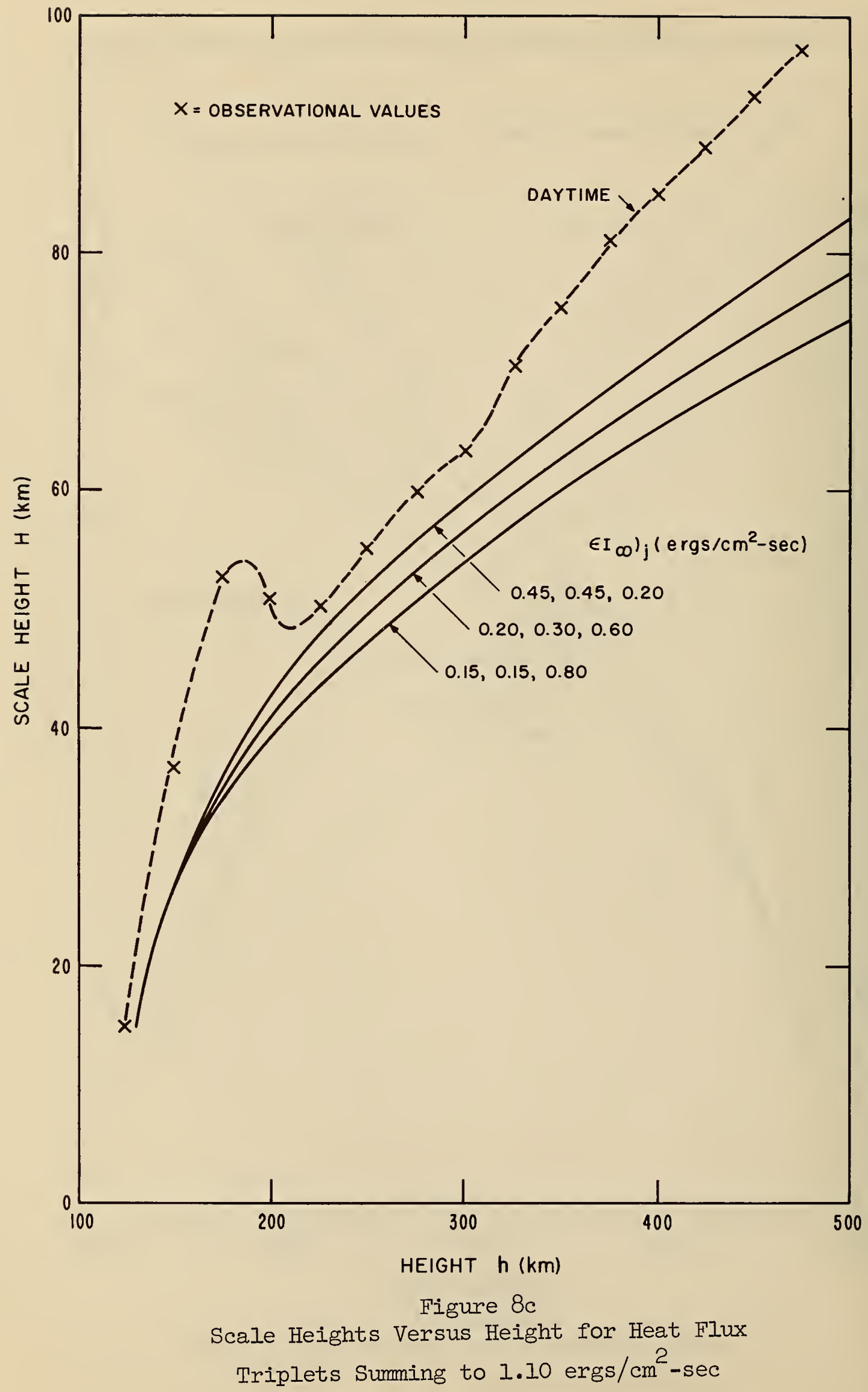




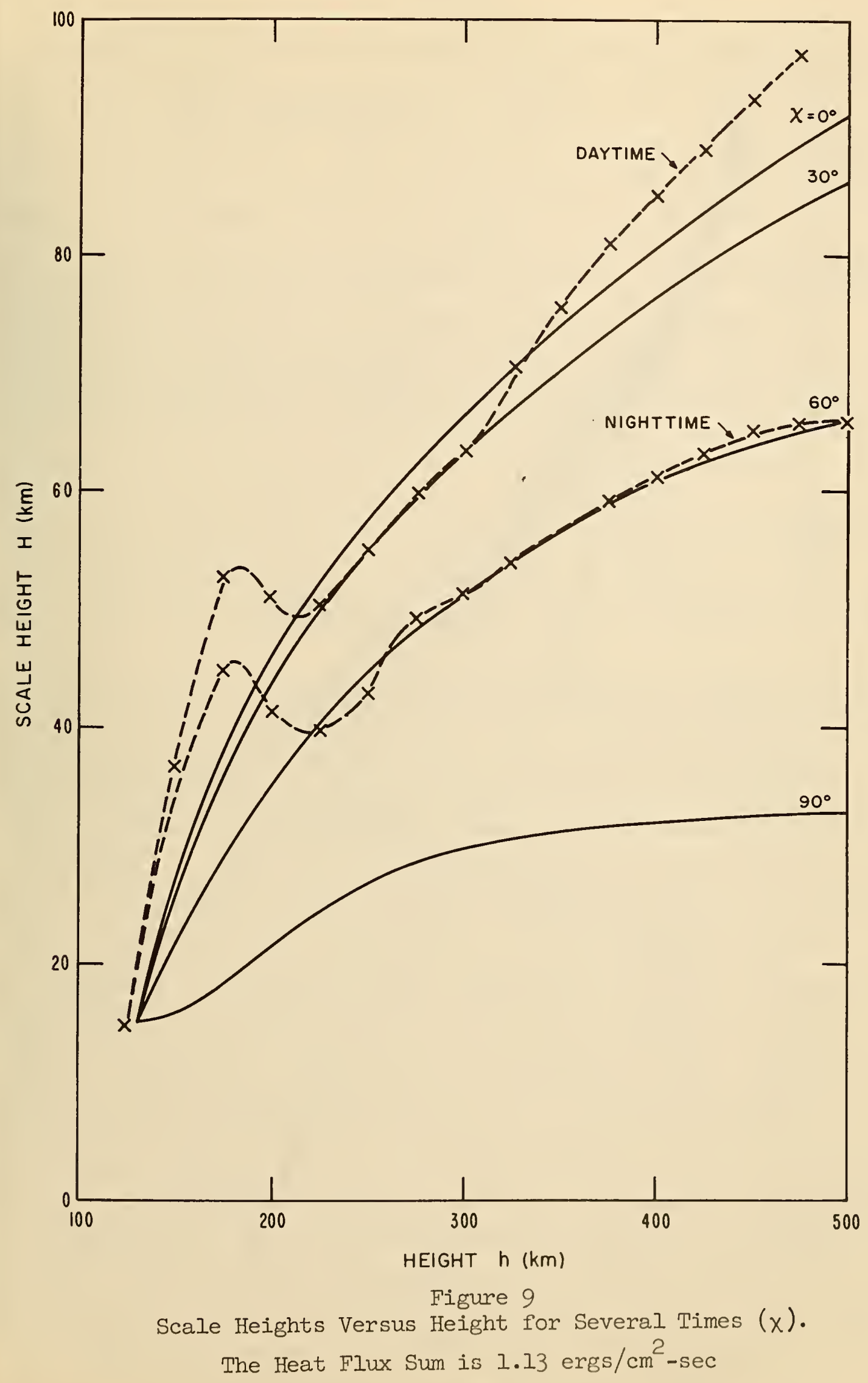


40

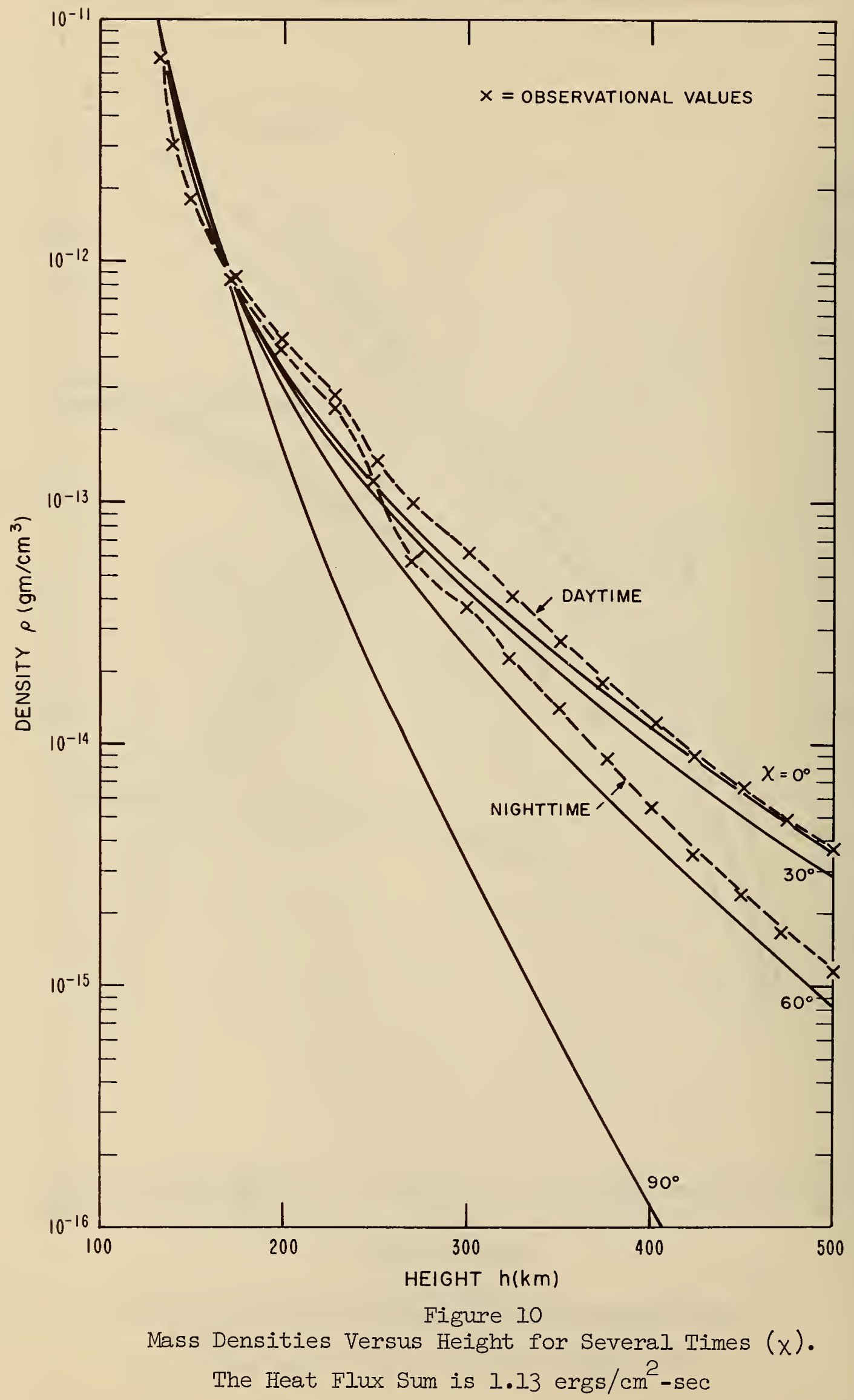


and VanZandt's slightly different boundary conditions; it is also due to their assumption of a pure oxygen atmosphere which increases thermal conduction. It is also seen that the dependence of $\epsilon I_{\infty}$ on $\lambda$ which is chosen is not very great. Thus a heat flux spectrum is chosen using: (1) figure 3 to get the relative values of $I_{\infty}$ in the three wave length ranges, (2) the expression for $\epsilon(\lambda)$ that $\epsilon(\lambda)=6 /(\mathrm{hc} / \lambda)[\mathrm{e} \cdot \mathrm{v} \cdot]$, and (3) a total heat flux sum of $1.13 \mathrm{ergs} / \mathrm{cm}^{2}-\mathrm{sec}$. This heat flux sum seems to fit the observed daytime densities best when an $\in I_{\infty}-$ versus $-\lambda$ profile consistent with ( 1 ) and (2) is chosen, as is seen in figure 10. It is to be noted that there is of the order of \pm 25 percent error to be associated with the observational $\rho(h)$ and $H(h)$ curves. As can be estimated by comparing figures $7 \mathrm{a}, 7 \mathrm{~b}$, and $7 \mathrm{c}$ with figure 10 , this would lead to about $a \pm 10$ percent uncertainty in the estimate of the heat flux sum. In figures 9 to 23 a heat flux sum of $1.13 \mathrm{ergs} / \mathrm{cm}^{2}-\mathrm{sec}$ is used. The individual fluxes chosen are $\left.\left.\in I_{\infty}\right)_{304}=0.21 \mathrm{ergs} / \mathrm{cm}^{2}-\mathrm{sec}, \in I_{\infty}\right)_{584}=0.61$ ergs $/ \mathrm{cm}^{2}-\mathrm{sec}$, and $\left.\in I_{\infty}\right)_{900}=0.31 \mathrm{ergs} / \mathrm{cm}^{2}-\mathrm{sec}$. This choice implies $\left.I_{\infty}\right)_{304}$ $\left.=1.54 \mathrm{ergs} / \mathrm{cm}^{2}-\mathrm{sec}, I_{\infty}\right)_{584}=2.75 \mathrm{ergs} / \mathrm{cm}^{2}-\mathrm{sec}$, and $\left.I_{\infty}\right)_{900}=0.89$ ergs $/ \mathrm{cm}^{2}-\mathrm{sec}$ for a total F-region ionizing flux of $5.18 \mathrm{ergs} / \mathrm{cm}^{2}-\mathrm{sec}$.

Figure 9 shows scale heights-versus-height for several values of $x$. Here it is seen that relatively good agreement is obtained between the observational scale heights and the $x=0^{\circ}$ theoretical profile. Above about $350 \mathrm{~km}$, the observational scale heights have a greater slope than the theoretical ones (indicating a considerable $\mathrm{M}(\mathrm{h})$ gradient), but this may be due to the inaccuracies in the observed scale heights at these heights. Figure 11 gives the temperature-versus-height profiles for several $X$ 's. The sharp temperature gradients near $130 \mathrm{~km}$ are due to the low conductivity there, necessitating high gradients to conduct away the large energy f.luxes caused by photonic heating in the region around $200 \mathrm{~km}$. At high heights there is little photonic heating or radiative loss, and so very little conduction is required. This results in approximate isothermality above about $300 \mathrm{~km}$. Figure 12 shows mass density-versus- $x$ at several heights. At $210 \mathrm{~km}$ Martin and Priester (1960) give an observational curve and this is compared. The form and degree of variation is quite similar up to about $x=50^{\circ}$. Figure 13 shows the temperature as a function of time for several heights. Figures 14-17 show the atmospheric composition as a function of height for several times. The $\mathrm{O}_{2}$ densities of Friedman (1960) are shown from 130-200 $\mathrm{km}$ and good agreement with these is obtained. The points from 160-200 $\mathrm{km}$ are $\mathrm{O}_{2}$ densities obtained near $\mathrm{x}=90^{\circ}$ and so may be slightly lower than noontime values. In this case the calculated $\mathrm{O}_{2}$ densities are somewhat low, indicating some mixing of $\mathrm{O}_{2}$ may occur above $130 \mathrm{~km}$. Near 130 $\mathrm{km}$, the slopes of the $\rho_{j}(\mathrm{~h})$ curves must be nearly the same since $\partial \mathrm{T} / \partial \mathrm{h}$ is 


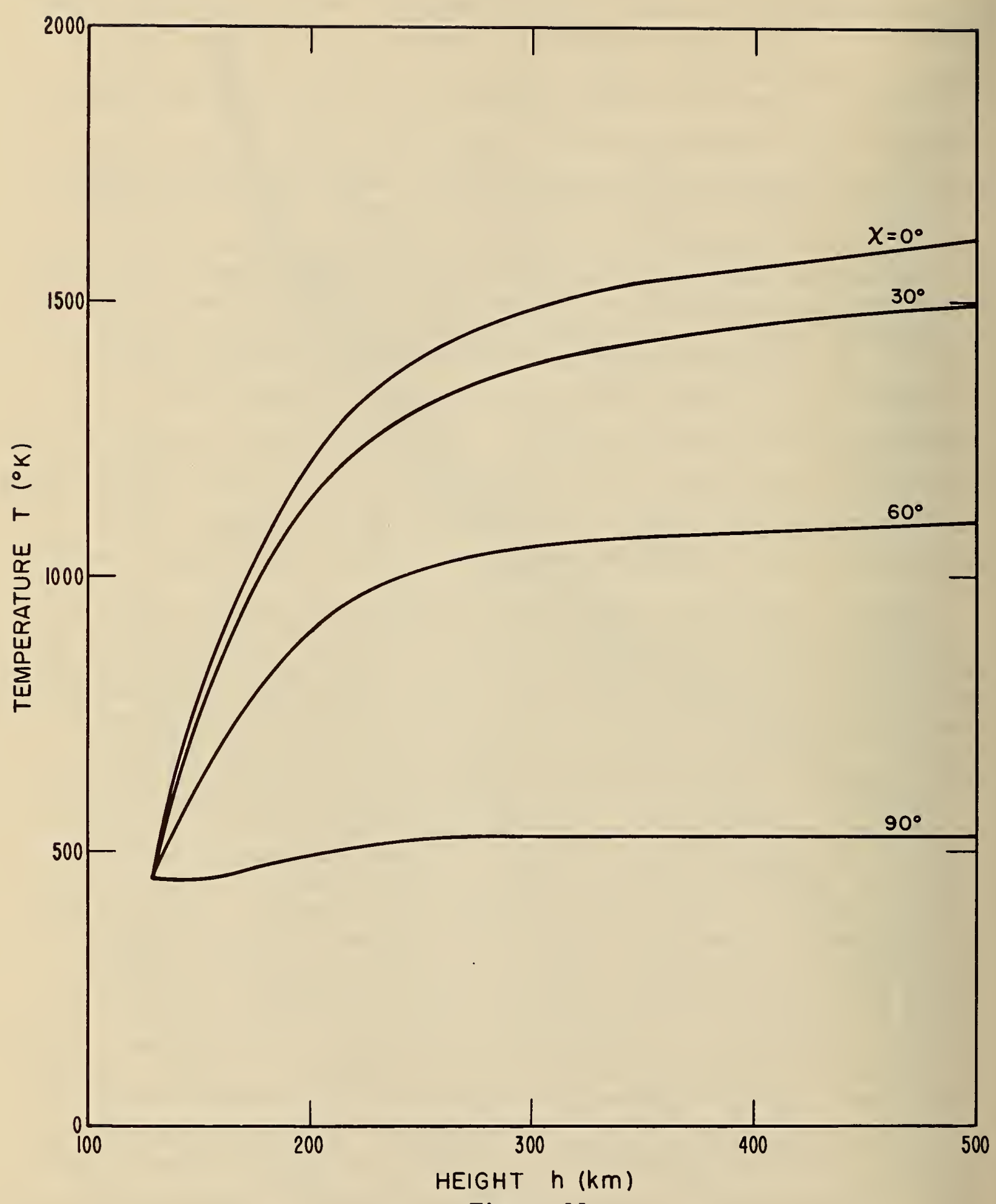

Figure 11

Temperature Versus Height for Several Times (x)

The Heat Flux Sum is $1.13 \mathrm{ergs} / \mathrm{cm}^{2}-\mathrm{sec}$ 
43

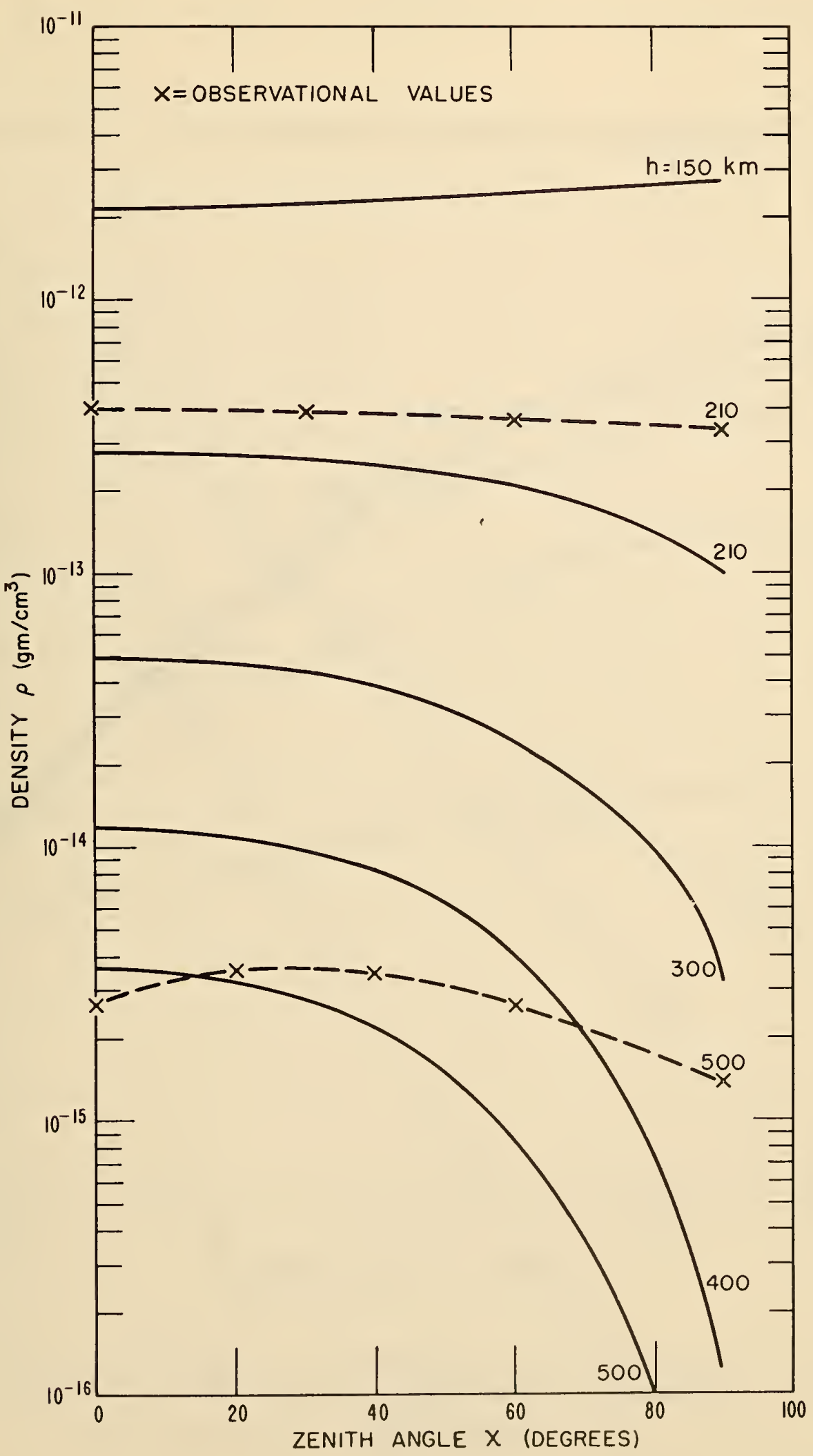

Figure 12

Mass Densities Versus Time $(x)$ for Several Heights. The Heat Flux Sum is $1.13 \mathrm{ergs} / \mathrm{cm}^{2}-\mathrm{sec}$ 


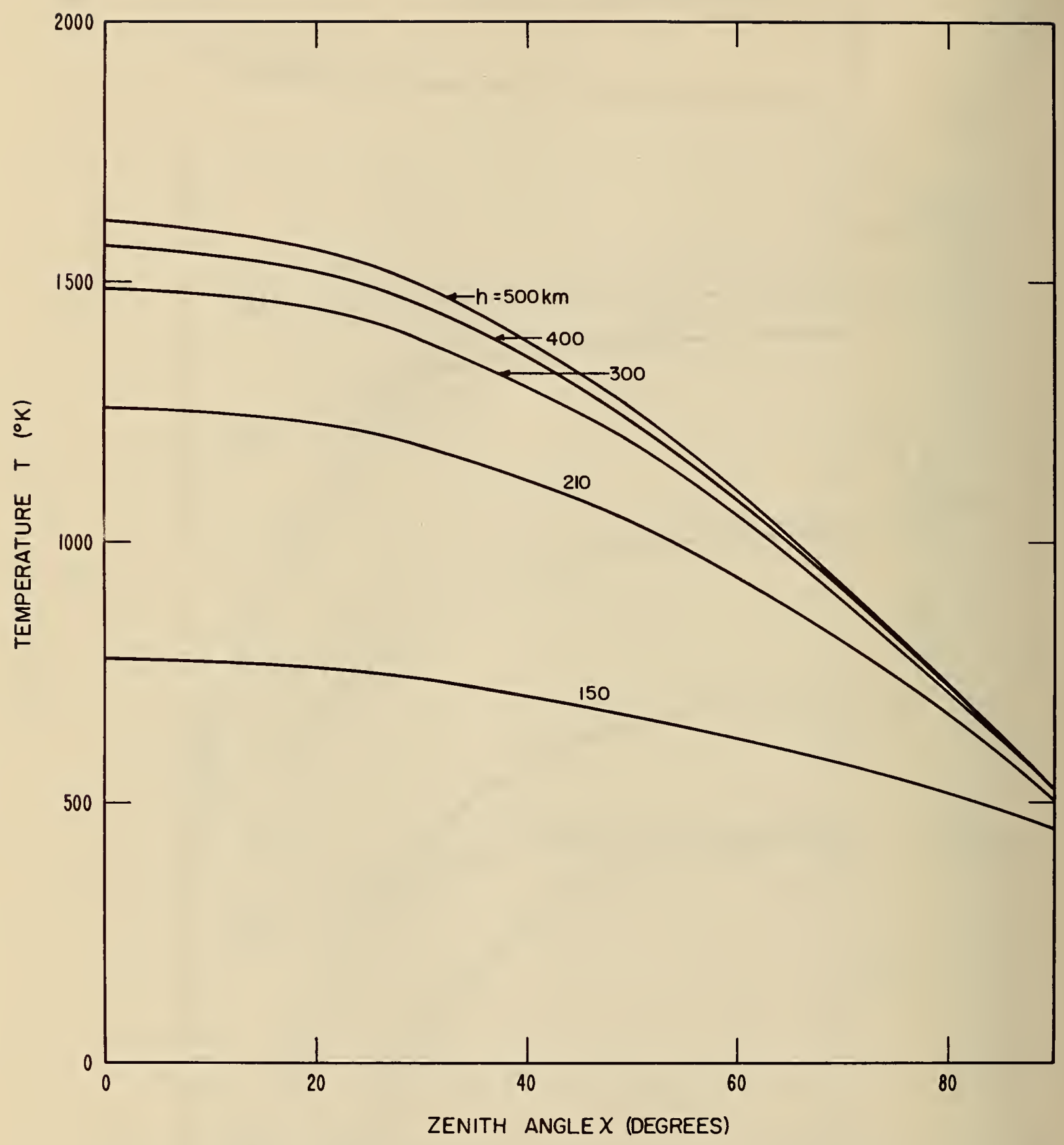

Figure 13

Temperature Versus Time $(x)$ for Several Heights. The Heat Flux Sum is $1.13 \mathrm{ergs} / \mathrm{cm}^{2}-\mathrm{sec}$ 


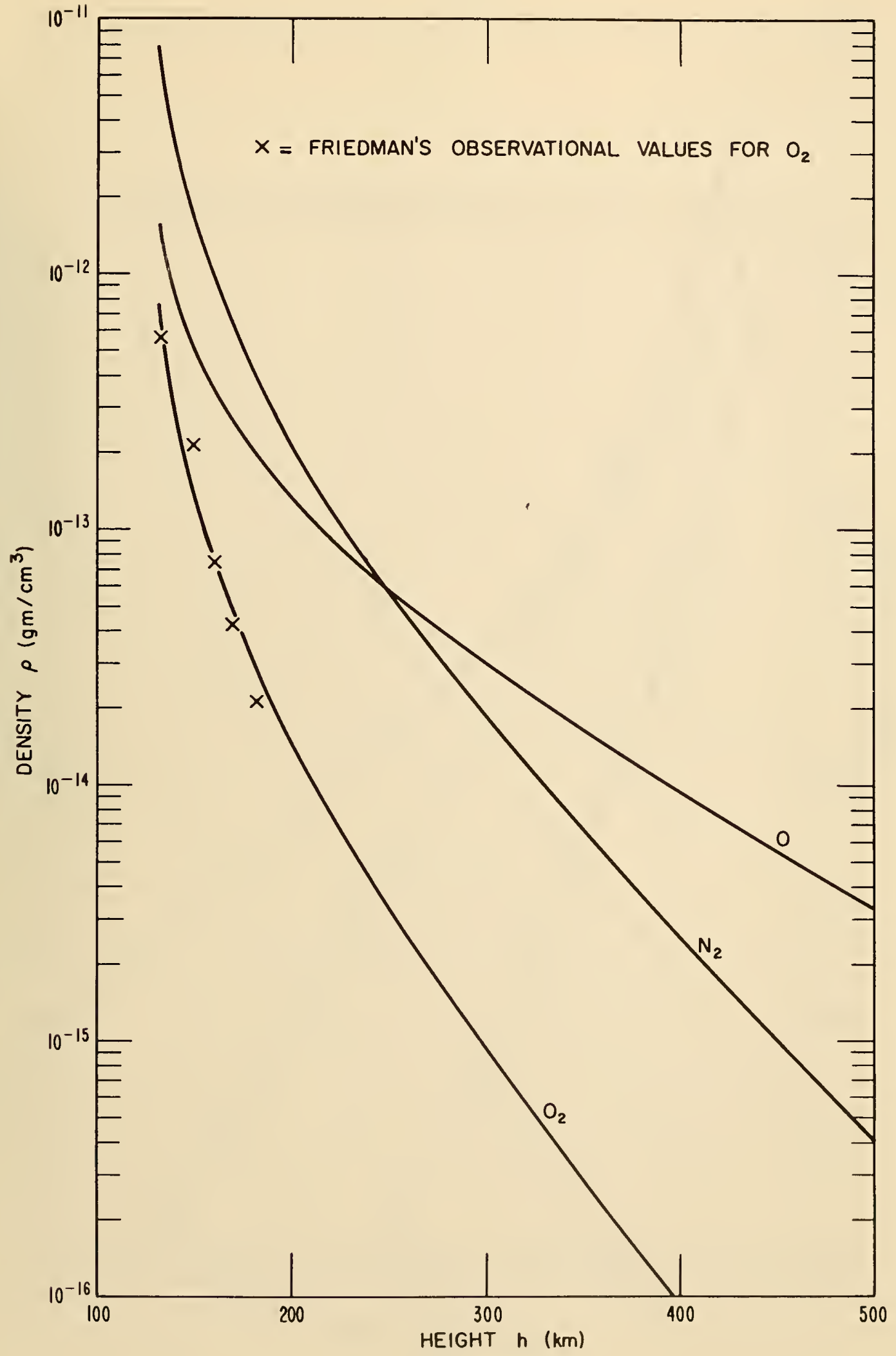

Figure 14

Detailed Composition Versus Height

For a Heat Flux Sum of $1.13 \mathrm{ergs} / \mathrm{cm}^{2}-\mathrm{sec}$ and $x=0^{\circ}$ 


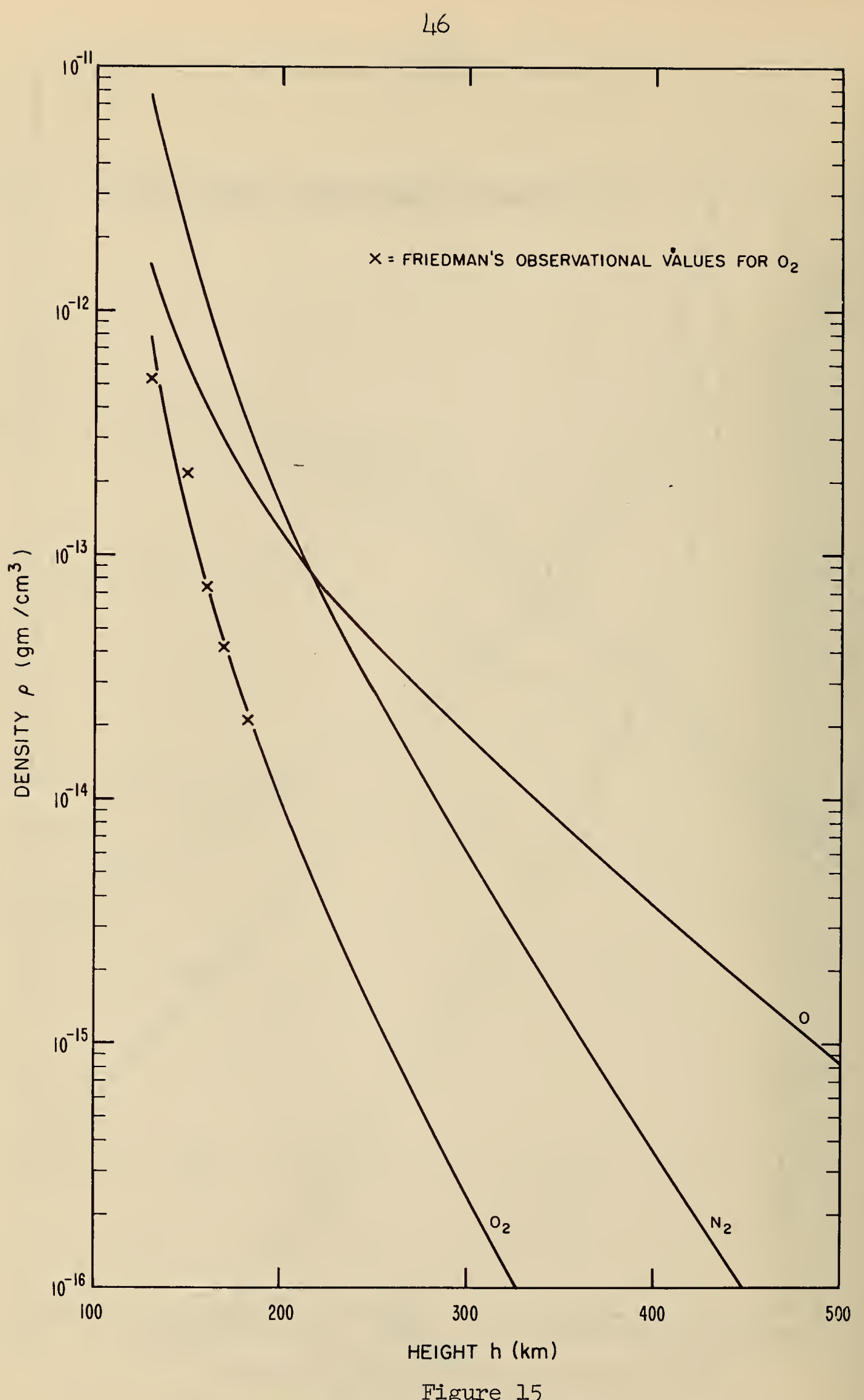

Detailed Composition Versus Height For a Heat Flux Sum of $1.13 \mathrm{ergs} / \mathrm{cm}^{2}-\mathrm{sec}$ and $\chi=30^{\circ}$ 


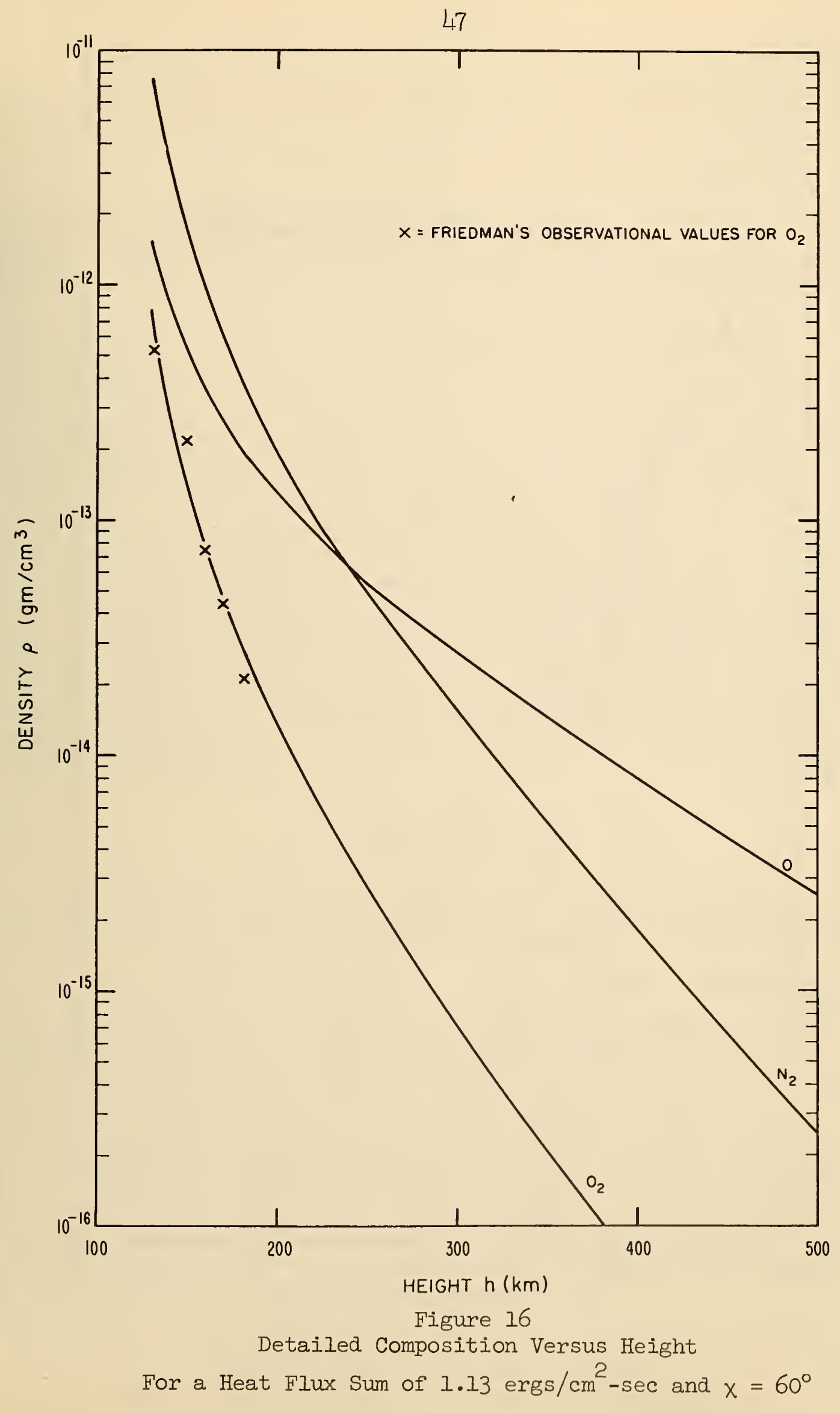




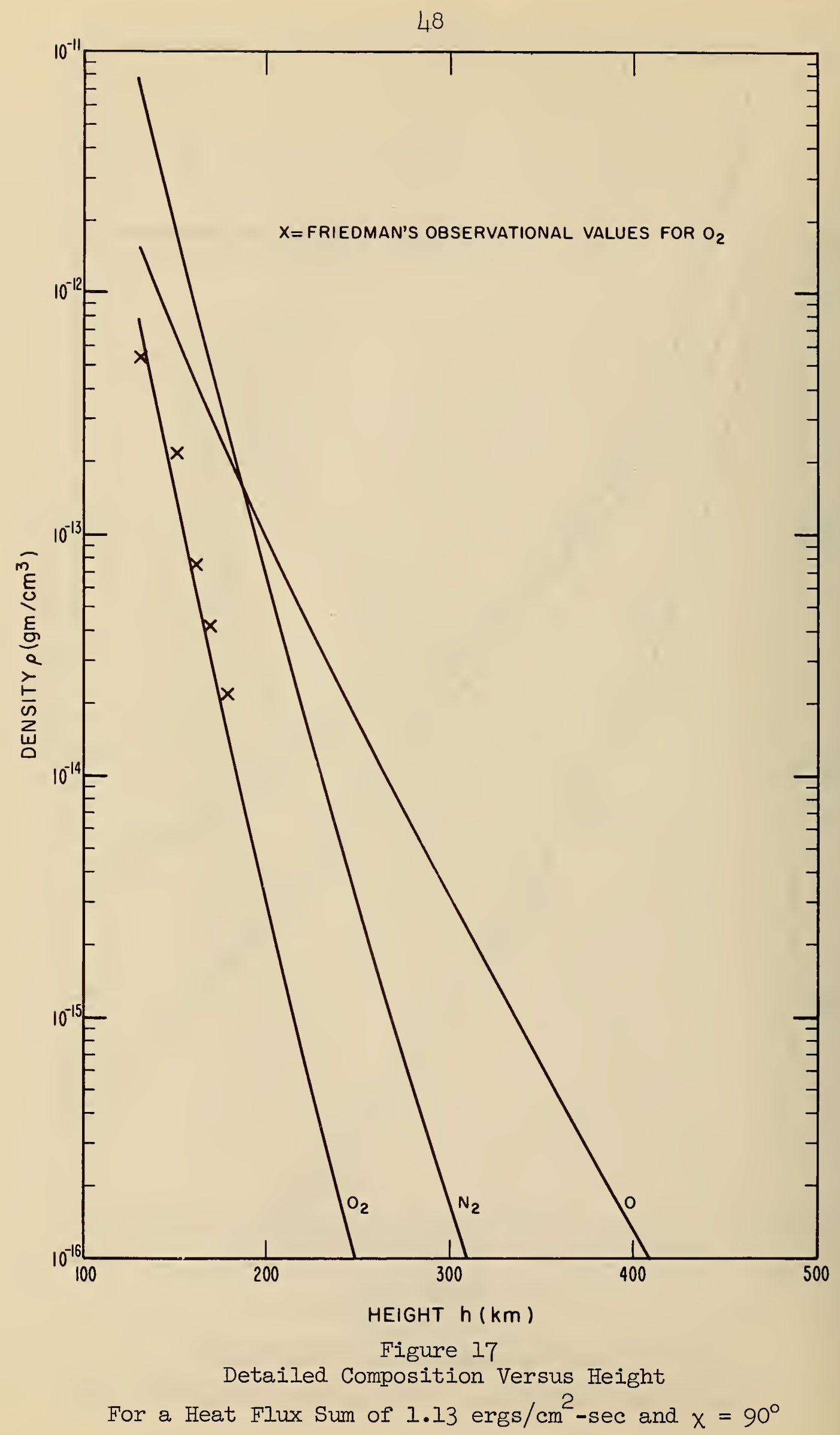


large. As $\partial \mathrm{T} / \partial H$ gets smaller (above the peak of production) the slopes of the $\rho_{j}(h)$ will begin to be characteristic of their individual scale heights. This effect causes $\rho_{\mathrm{N}_{2}}$ and $\rho_{0}$ not to cross until above $250 \mathrm{~km}$.

Figures 18-20 show the optical depths for the three wavelength ranges as a function of height for several times. At the peak of production, the optical depths are approximately $l+\partial H / \partial h$. Thus the production peak is at about $178 \mathrm{~km}$ for $\chi=0^{\circ}$. At high heights the optical depth's slope should become less as $\int_{h}^{h} p(h) d h$ approaches a pure exponential. The optical depths have been made to not go to zero at $500 \mathrm{~km}$ by using an $\mathrm{h}_{\mathrm{m}}$ of $510 \mathrm{~km}$ in their calculation. This was felt to be more physically reasonable than having the optical depths go to zero at the upper boundary. Figure 21 shows the total rate of heat generation-versus-height for several times. The $\chi=0$ peak of production is at about $178 \mathrm{~km}$ (as predicted by the optical depth curves) and because of the increased absorption path length, increases with $\chi$ to about $215 \mathrm{~km}$ at $\chi=90^{\circ}$. Figure 22 shows the radiative loss rate as a function of height for several times. It is seen that the radiative loss rate becomes increasingly important at lower heights compared to the gain rate (figure 2l). At the lower heights, however, other gains become important. Figure 23 shows the thermal conductivity-versus-height for $\chi=0^{\circ}$ and the molecular weight as a function of height for $x=0^{\circ}$. The thermal conductivity is seen to change by a factor of only about 2.4 over the F-region and the molecular weight by a factor of about 1.8 .

\section{b. Non-steady State}

Here only the first approximation was computed (this was done for the afternoon case when $\partial \mathrm{T} / \partial t$ is negative). Thus the results have more qualitative than quantitative value. The heat fluxes used are $\left.\in I_{\infty}\right)_{304}$ $=0.67 \mathrm{ergs} / \mathrm{cm}^{2}-\mathrm{sec}$ and $\left.\epsilon I_{\infty}\right)_{584}=0.33 \mathrm{ergs} / \mathrm{cm}^{2}-\mathrm{sec}$. The quasi-steady state is assumed to be valid at noon, which is necessarily true in the first approximation; also the temperature at $130 \mathrm{~km}$ is taken to be $420^{\circ} \mathrm{K}$ and to remain constant in time. The diffusive height is taken as before at $130 \mathrm{~km}$ and 100 percent dissociation of $\mathrm{O}_{2}$ is assumed at $h_{d}$. In figures 24 and 25 the mass densities and temperatures are shown as a function of height for several times during the afternoon. A fairly good fit to the observational densities is achieved for $x \approx 20^{\circ}$. In figures 26 and 27 the variation with $x$ of total mass density and temperature is shown for several heights. The afternoon density maximum is seen to occur at about $\chi=50^{\circ}$ in contrast to the observed densities (shown by dashed lines in figures 26 and 27) which peak at about $x=25^{\circ}$. 


\section{0}

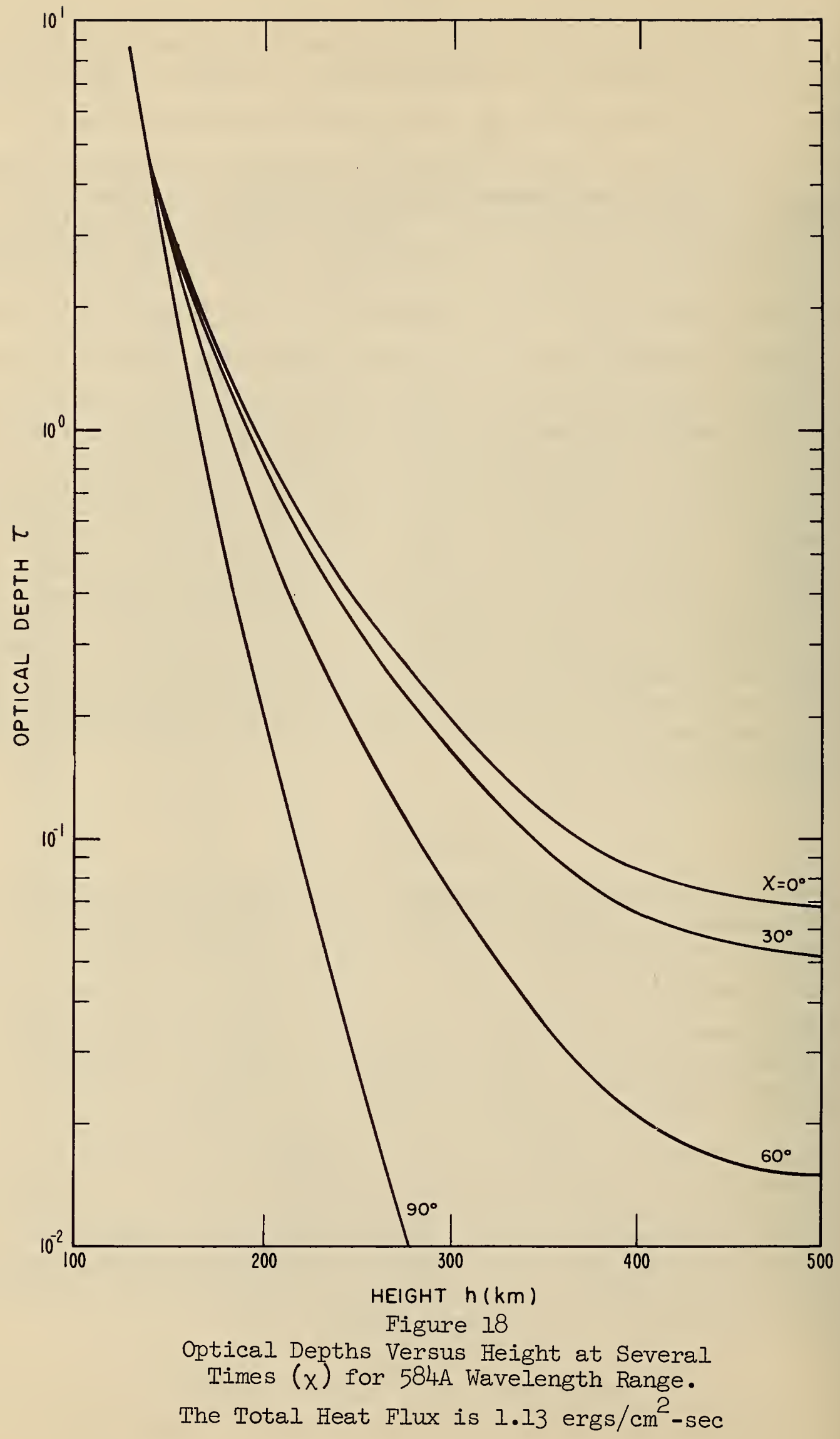




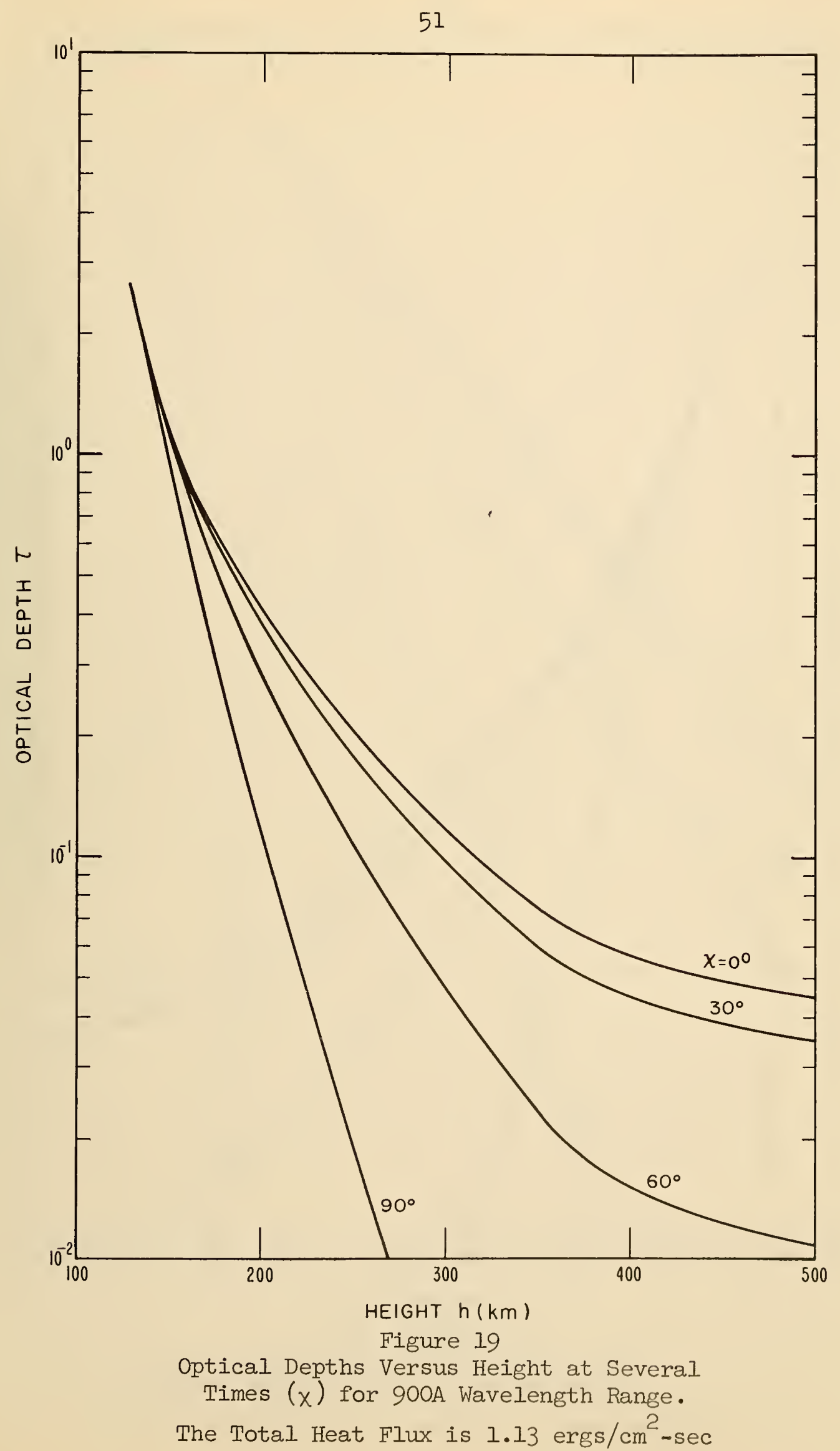


52

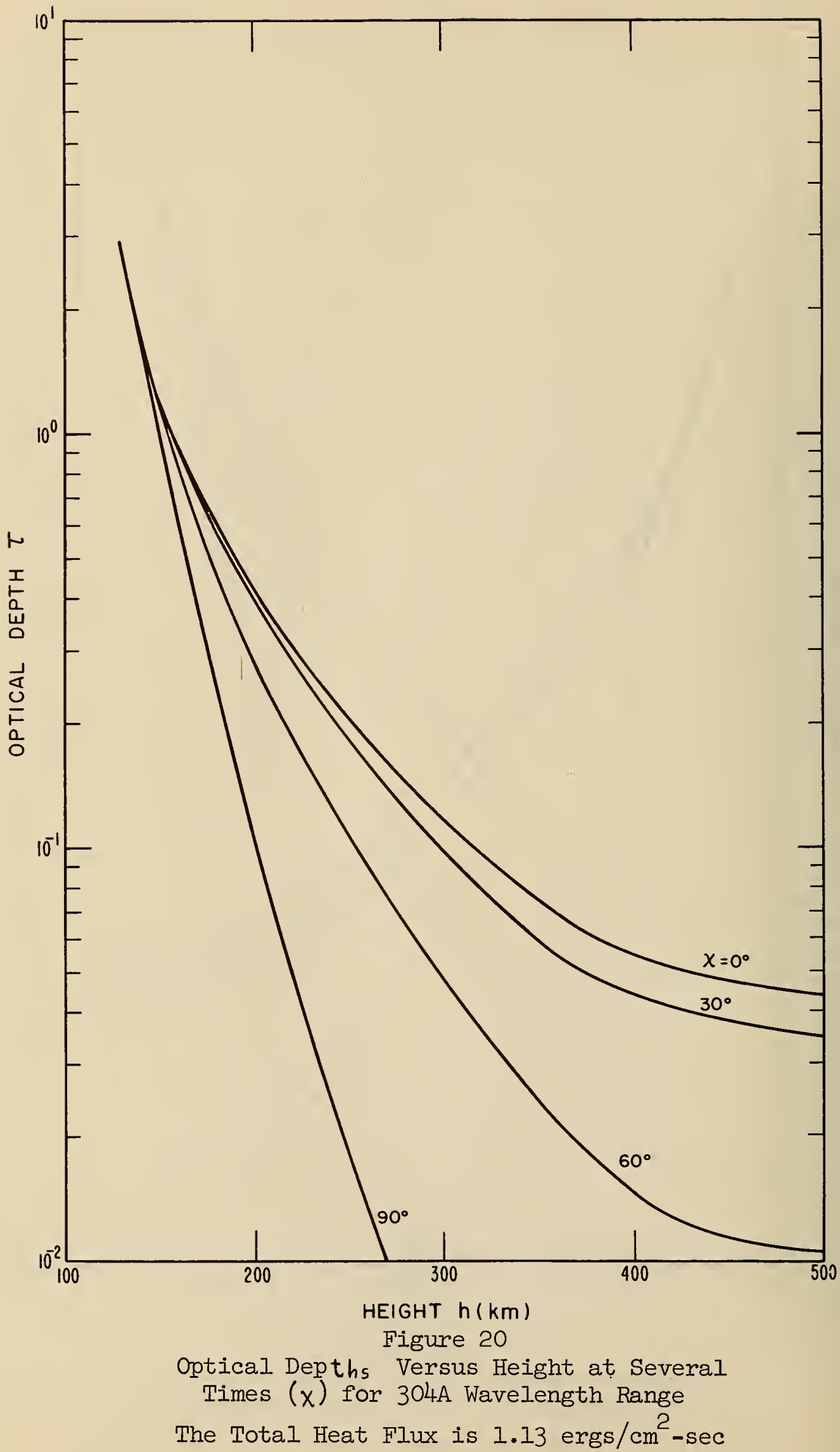




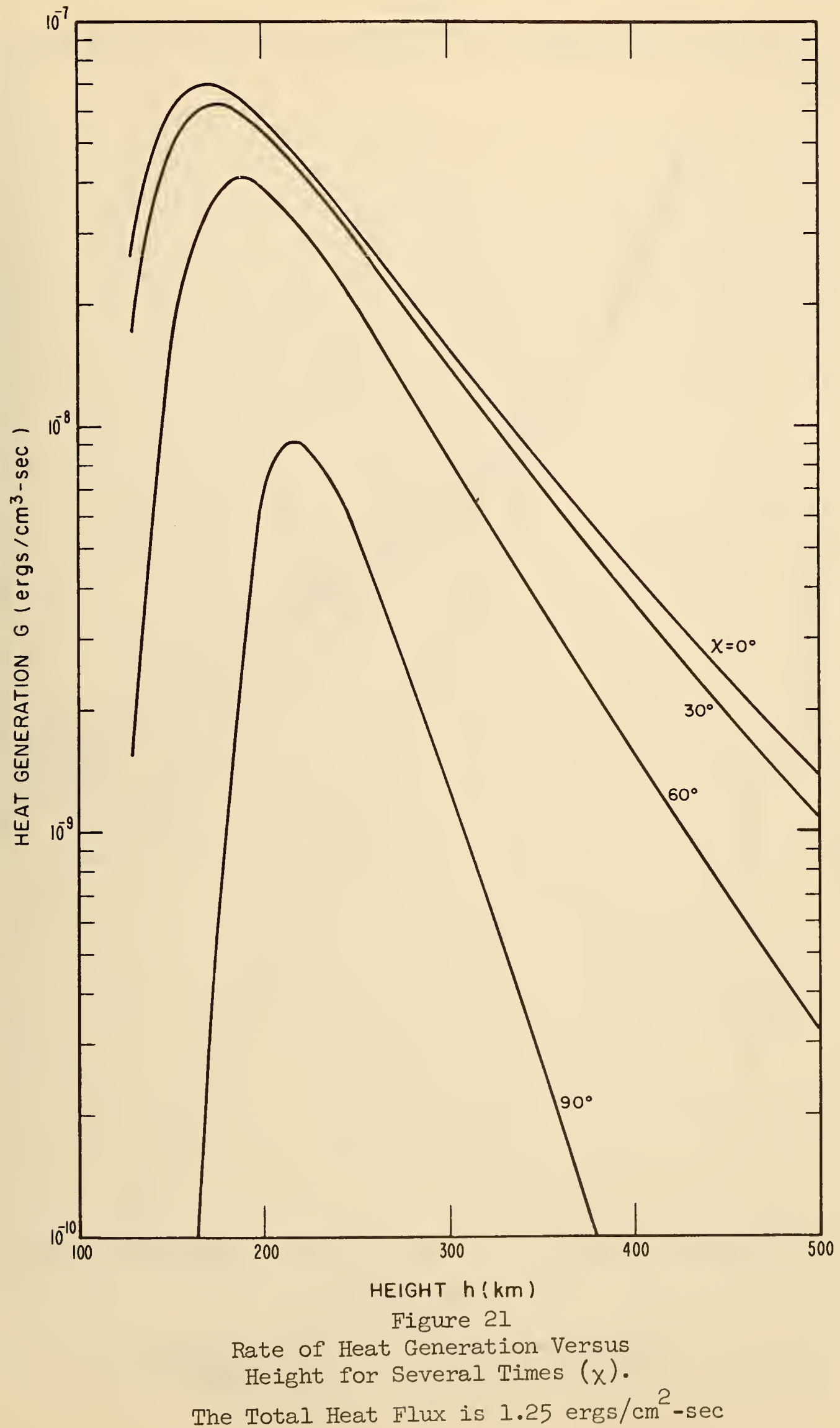




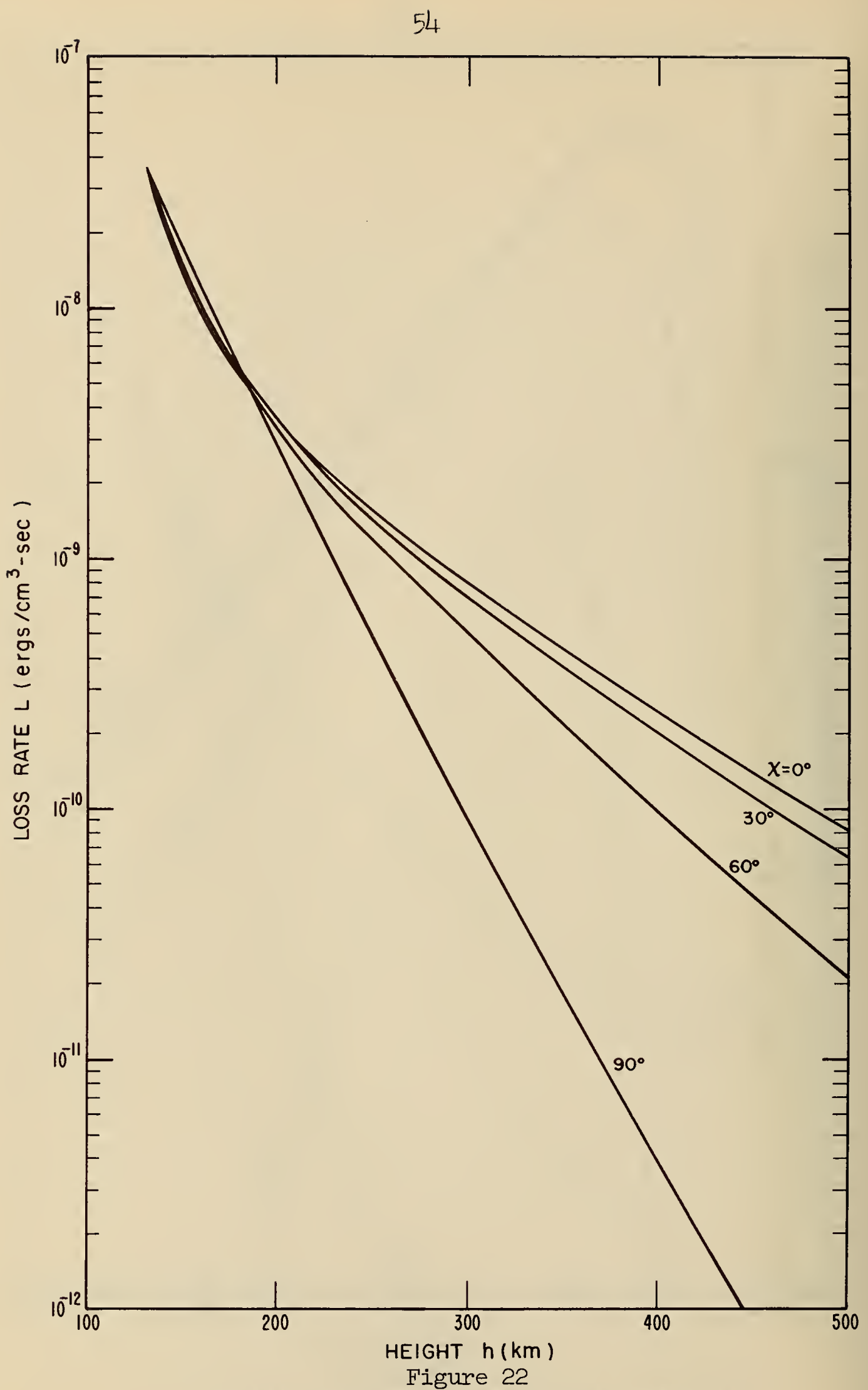

Radiative Heat Loss Rate Versus Height for Several Times $(x)$. The Total Heat Flux is $1.25 \mathrm{ergs} / \mathrm{cm}^{2}-\mathrm{sec}$ 


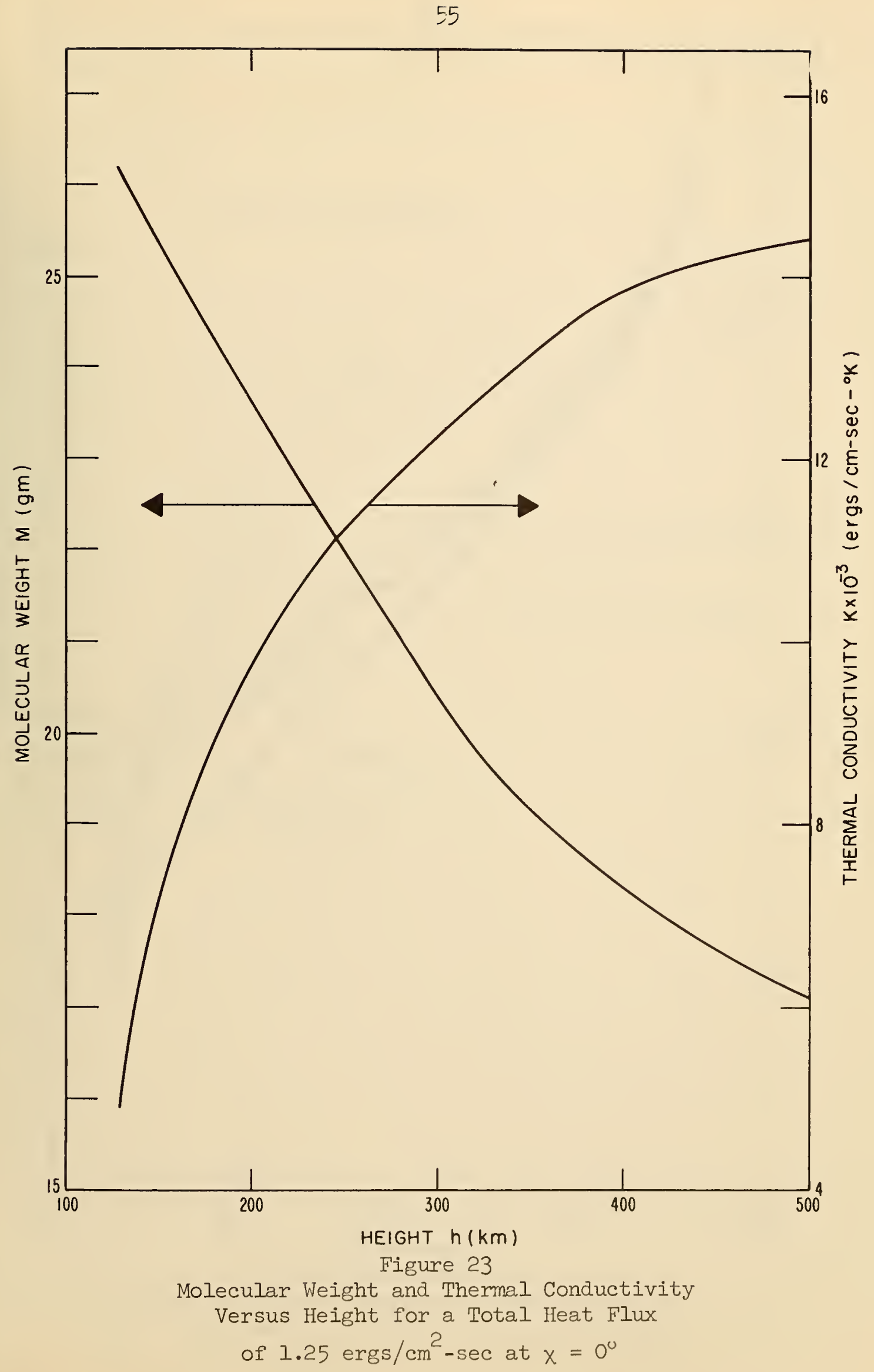


56

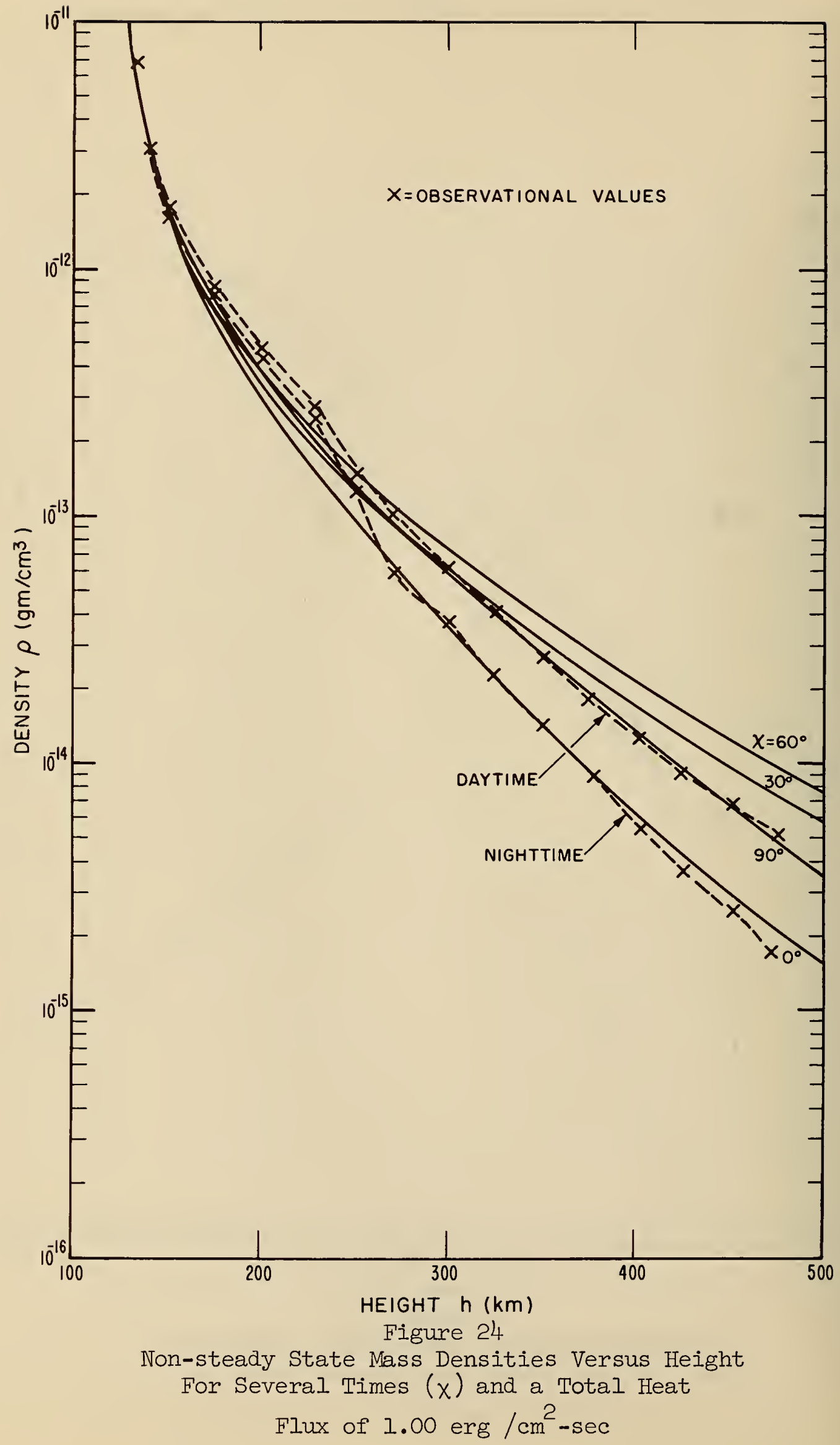




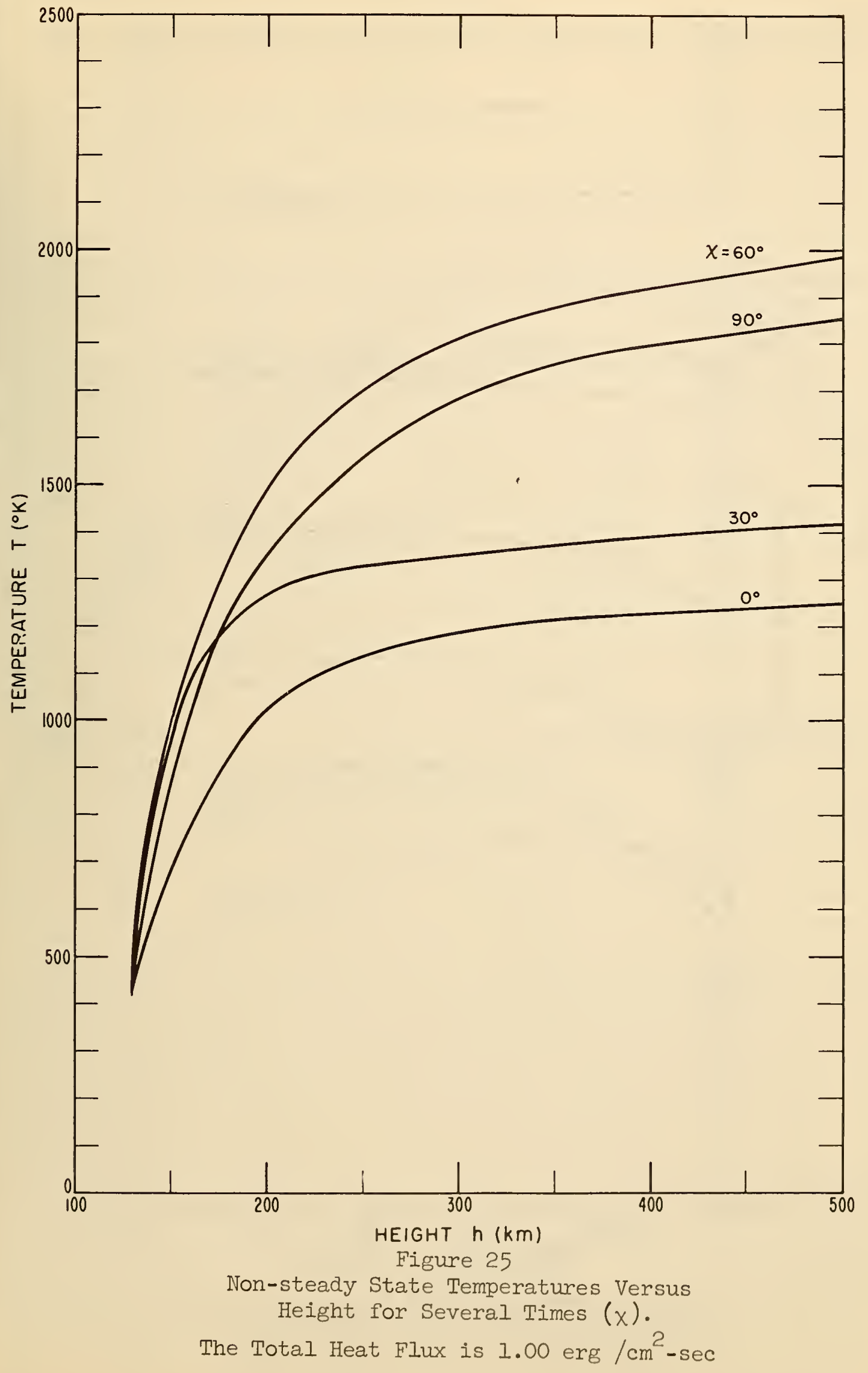




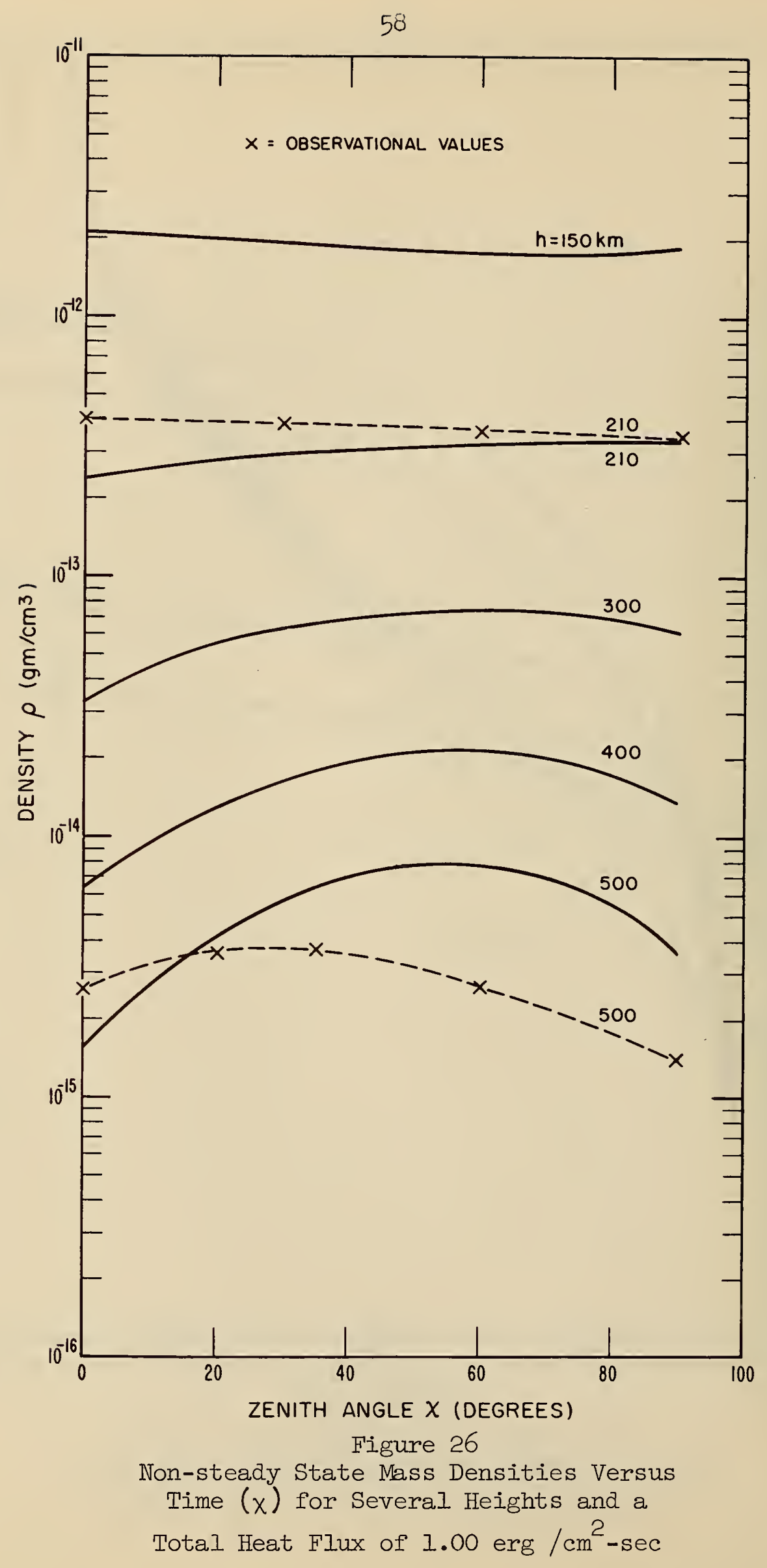




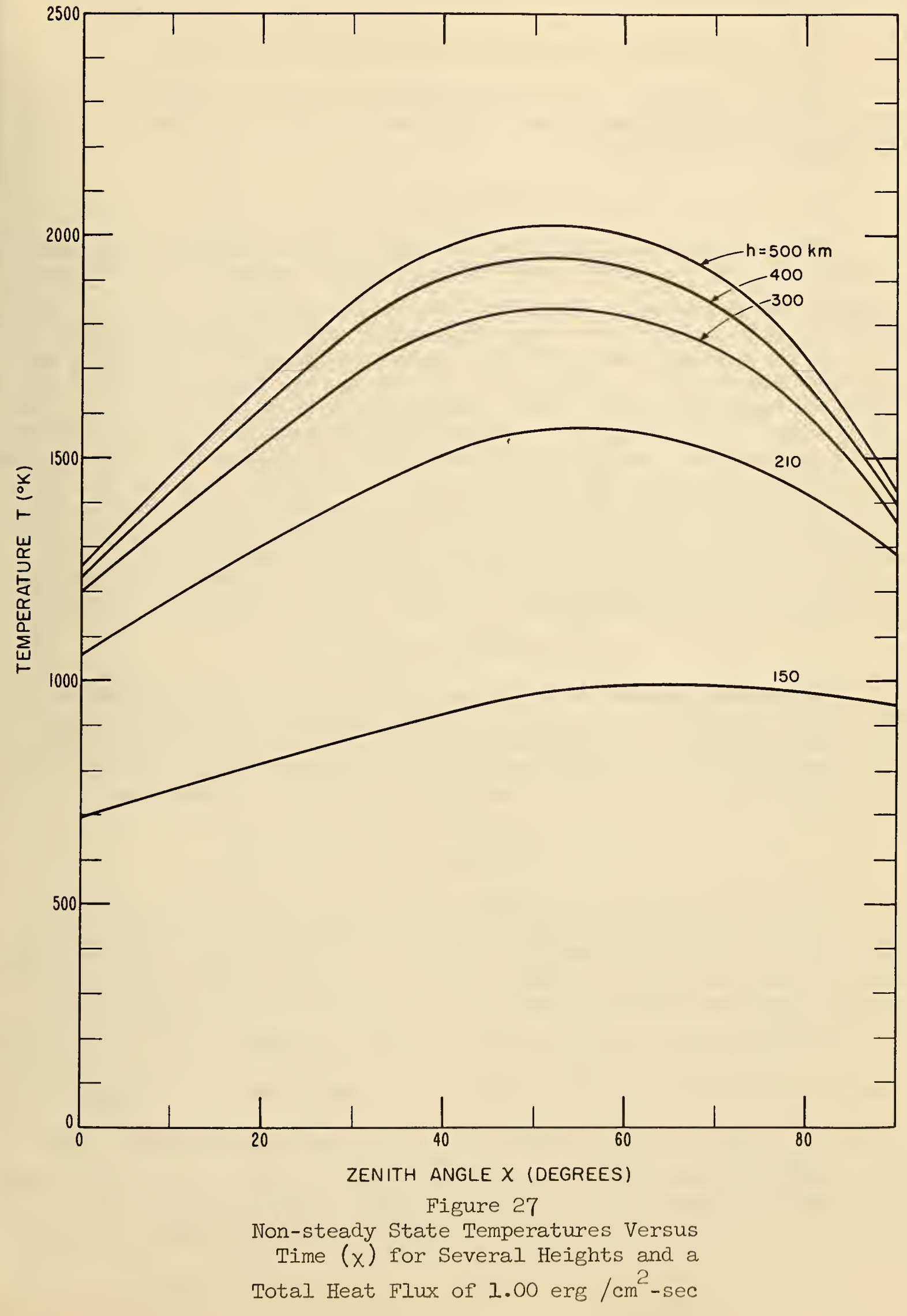


5.2 Boundary Conditions and Parameter Variation

In this discussion all of the variations, unless otherwise stated, refer to the chosen quasi-steady state model for $x=0^{\circ}$. The following variations are considered:

\section{a. Scale Height at $130 \mathrm{~km}$}

The boundary scale height may be varied if the boundary molecular weight (i.e., the $h_{\mathrm{d}}$ and degree of dissociation chosen) or the boundary temperature is changed. It may also be varied by changing $T$ and $M$ together. Only the first two variations are treated here. The effect of varying the boundary temperatures is shown in figure 29. This is a rather serious variation. The effect of varying the molecular weight is shown in figure 28 where a pure 0 atmosphere with $\mathrm{T}(130)=450^{\circ} \mathrm{K}$ is compared with the chosen model. The variation is seen not to be as serious since a pure oxygen atmosphere fairly well approximates the thermal situation in the F-region. The variation in $\rho(h)$, if $\mathrm{H}(130)$ is increased to $22 \mathrm{~km}$ (probably a more physically realizable situation) instead of decreased to $8 \mathrm{~km}$, will be of about the same magnitude and in the opposite sense. The scale height at $130 \mathrm{~km}$ is perhaps uncertain by about \pm 20 percent. Thus the resultant density curves may be uncertain as much as \pm 30 percent at high heights due to these types of variation of $\mathrm{H}(130)$.

\section{b. Boundary Density}

If the scale height at $130 \mathrm{~km}$ is kept fixed at $15 \mathrm{~km}$ and the boundary density varied, the resultant density variation is shown in figure 30. The boundary density has considerable effect on the resultant density profiles. The boundary density is uncertain by about \pm 20 percent. This makes the density profile uncertain by about the same amount.

\section{c. Choice of Absorption Cross Sections}

The effect of doubling and halving the absorption cross sections used on the computed densities is shown in figure 31 . It is seen that the density profiles are rather insensitive to this variation. This is because near the lower boundary most of the incident electromagnetic radiation has been absorbed even if the $A_{j i}$ are made considerably less. Thus the temperature gradients near the lower boundary will be high since they are determined by $\int_{h}^{h} m(G-L) d h$. This means most of the temperature rise will occur before the change in the $A_{j i}$ becomes effective. Thus the temperature profiles will not be significantly altered and the resultant density versus height curves will also be change $\vec{i}$ very little. It is to be noted here that if all the $A_{j i}$ and $\rho_{i}(130)$ are changed in the 


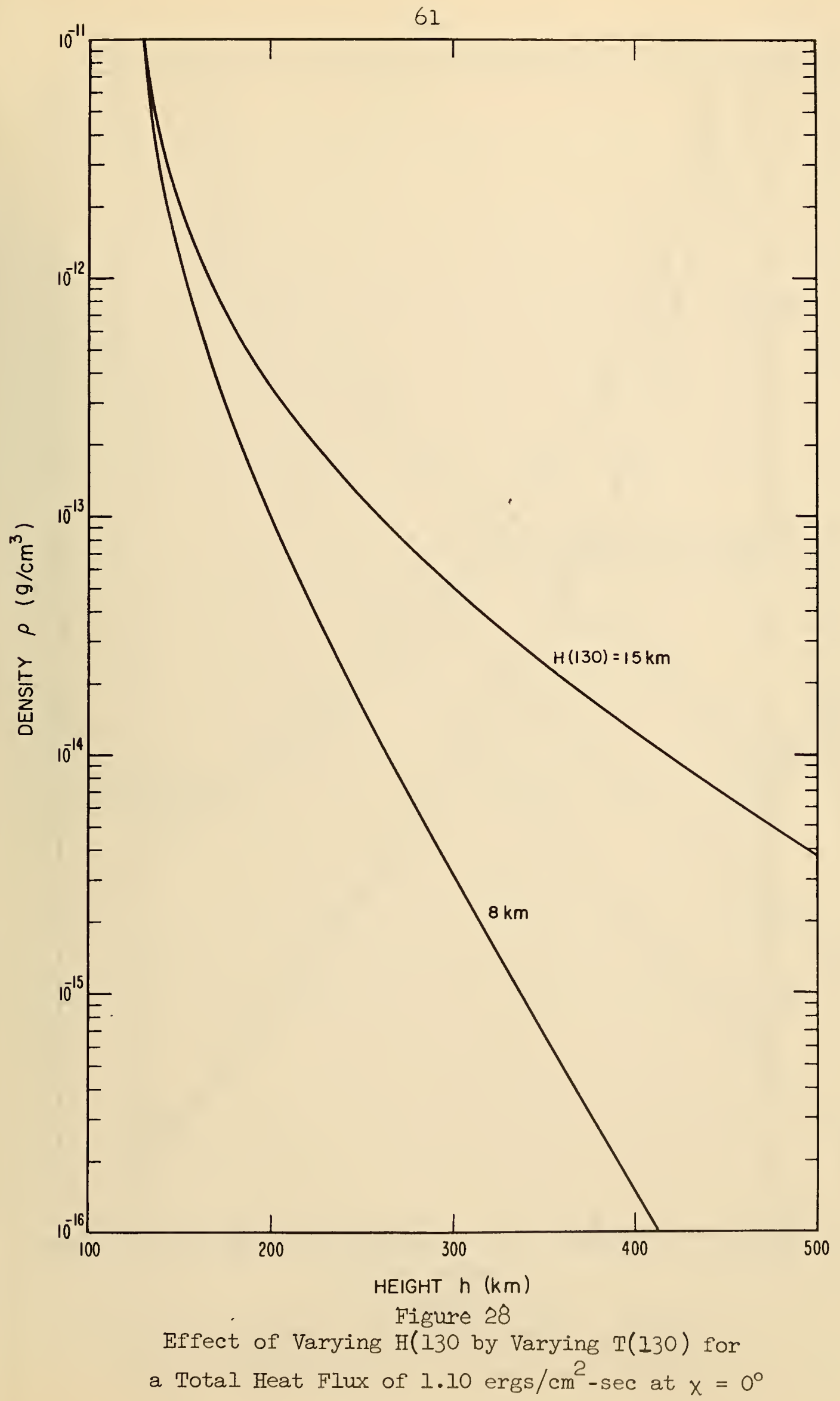




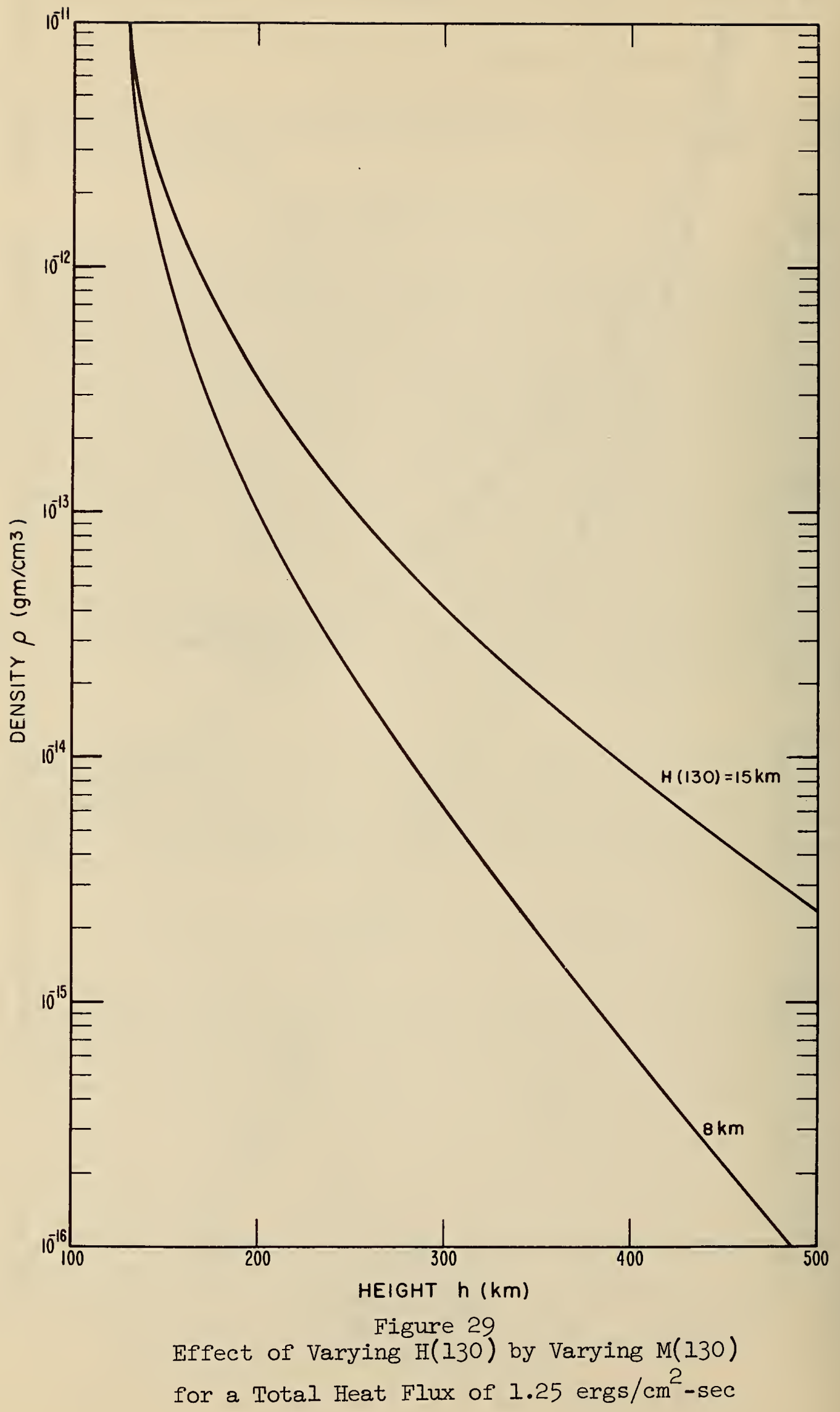




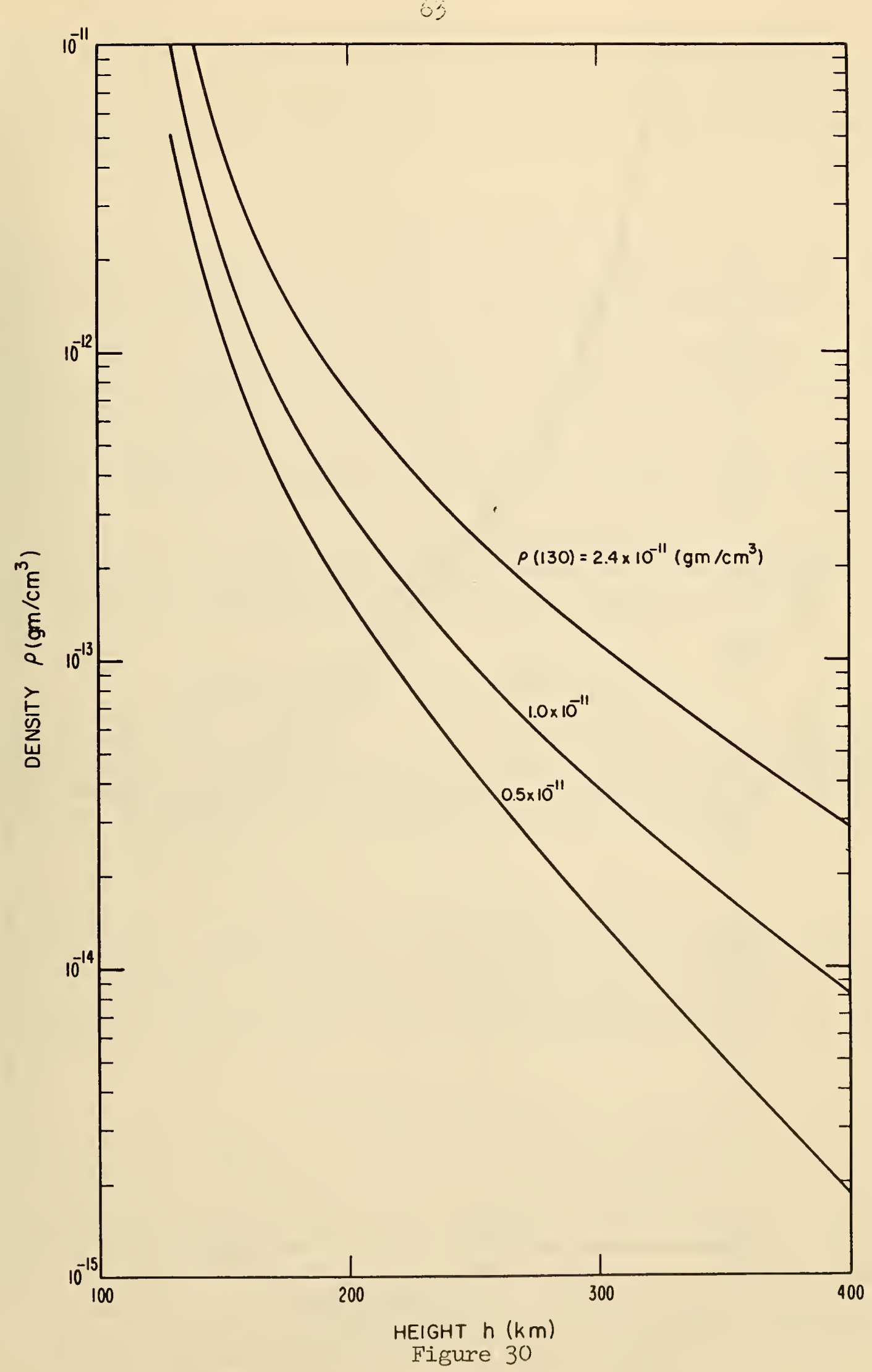

Effect of Varying Boundary Mass Density.

The Total Heat Flux is $1.25 \mathrm{ergs} / \mathrm{cm}^{2}-\mathrm{sec}$ and $x$ is $0^{\circ}$ 
64

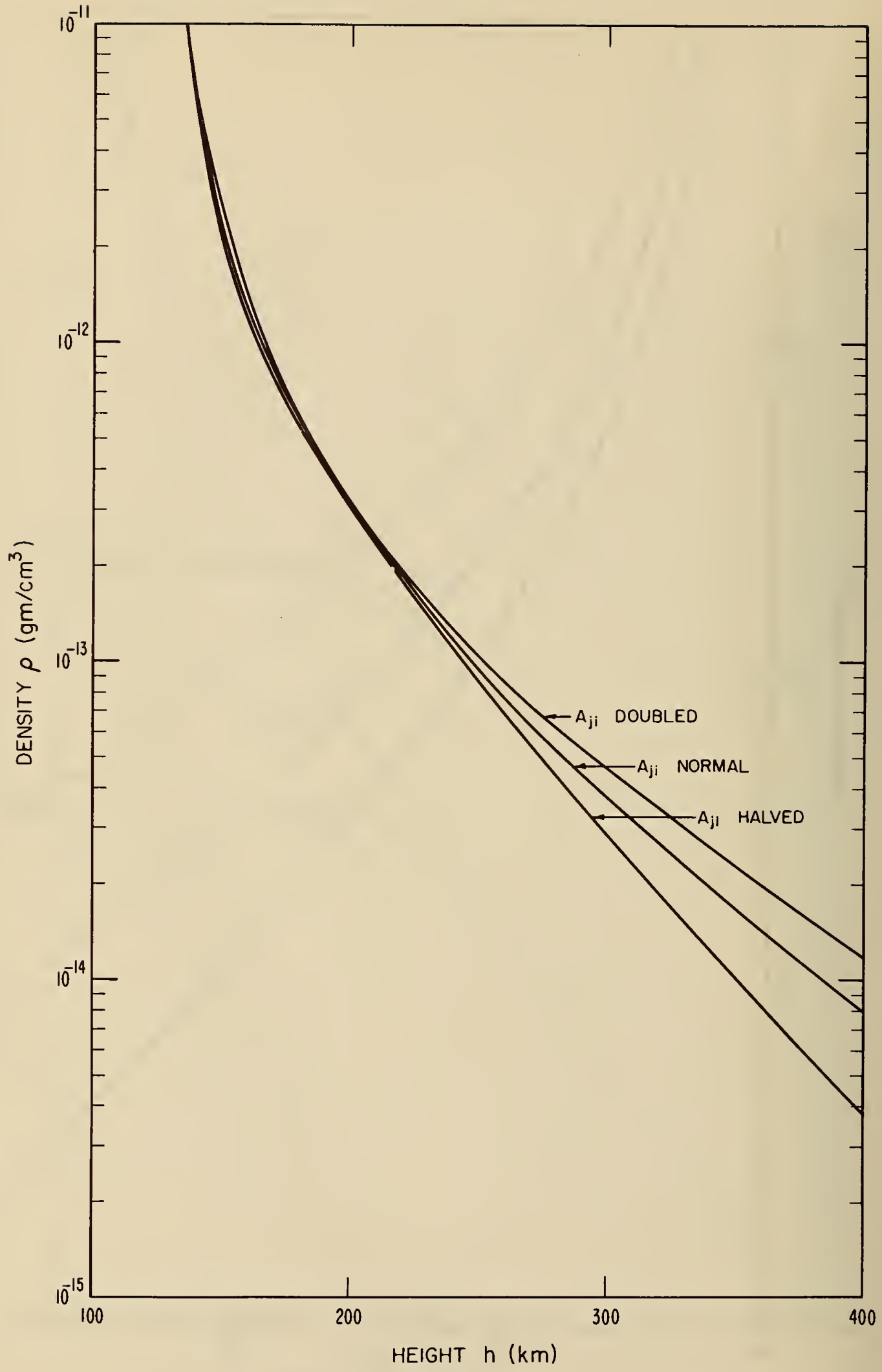

Figure 31

Effect on $\rho(h)$ of Varying Cross Sections for

a Total Heat Flux of $1.25 \mathrm{ergs} / \mathrm{cm}^{2}-\mathrm{sec}$ at $\chi=0^{\circ}$ 
same manner, such that the products $A_{j i} \rho_{i}(130)$ in (4-4) are unchanged, the resultant density profiles will merely be multiplied by the factor by which the $\rho_{i}(130)$ were changed.

\section{d. The Effect of $h_{d}$}

The scale height and the degree of dissociation at 130 is kept fixed to investigate this variation. The degree of dissociation of $\mathrm{O}_{2}$ at $130 \mathrm{~km}$ is kept fixed by picking the degree of dissociation at the $\mathrm{h}_{\mathrm{d}}$ in question such that an extrapolation to $130 \mathrm{~km}$ (assuming diffusive equilibrium to hold between the chosen $h_{d}$ and $130 \mathrm{~lm}$ and picking a suitable temperature model in this range) gives 80 percent dissociation there. The total densities are plotted versus height in figure 32 for several $h_{d}$ from $110-150 \mathrm{~km}$. The effect of $h_{d}$ is, seen to be small on the total mass densities. The effect is also relatively small on the temperatures and somewhat more in the scale heights because of the variation of $M(h)$. The effect on the detailed composition is, as previously mentioned, quite large. A lower $h_{d}$ implies more $\mathrm{O}_{2}$ and $\mathrm{O}$ and less $\mathbb{N}_{2}$.

\section{e. The Variation of the Total Heat Flux}

For heat fluxes of $3.3,1.7,1.1$, and $0.3 \mathrm{ergs} / \mathrm{cm}^{2}-\mathrm{sec}$, total mass densities versus heights are plotted in figure 33. T'he mass density profile is seen to be very sensitive to this parameter since the total rate of heat generation, $G(r, t)$, is directly proportional to $\Sigma \in I_{\infty}$.

\section{f. The Variation of Thermal Conductivity}

The effect of increasing the conductivity by 50 percent is shown in figure 34 compared with the total mass density profile for the chosen thermal conductivity. The density profiles are quite sensitive to this quantity since the conductive flux is directly proportional to thermal conductivity.

\section{g. The Effect of $\mathrm{F}_{\mathrm{m}}$}

The density profiles for several values of $\mathrm{F}_{\mathrm{m}}$ are shown in figure 35. The value of $\mathrm{F}_{\mathrm{m}}$ of $0.575 \times 10^{17} \mathrm{ergs} / \mathrm{sec}$ is just that required to maintain the nighttime scale height at $500 \mathrm{~km}$. The temperature and thus the scale height is relatively insensitive to $\mathrm{F}_{\mathrm{m}}$ except at high heights where the photonic input is small compared to any coronal conduction. Thus figure 35 shows the mass density profile to be relatively insensitive to $F_{m}$. 


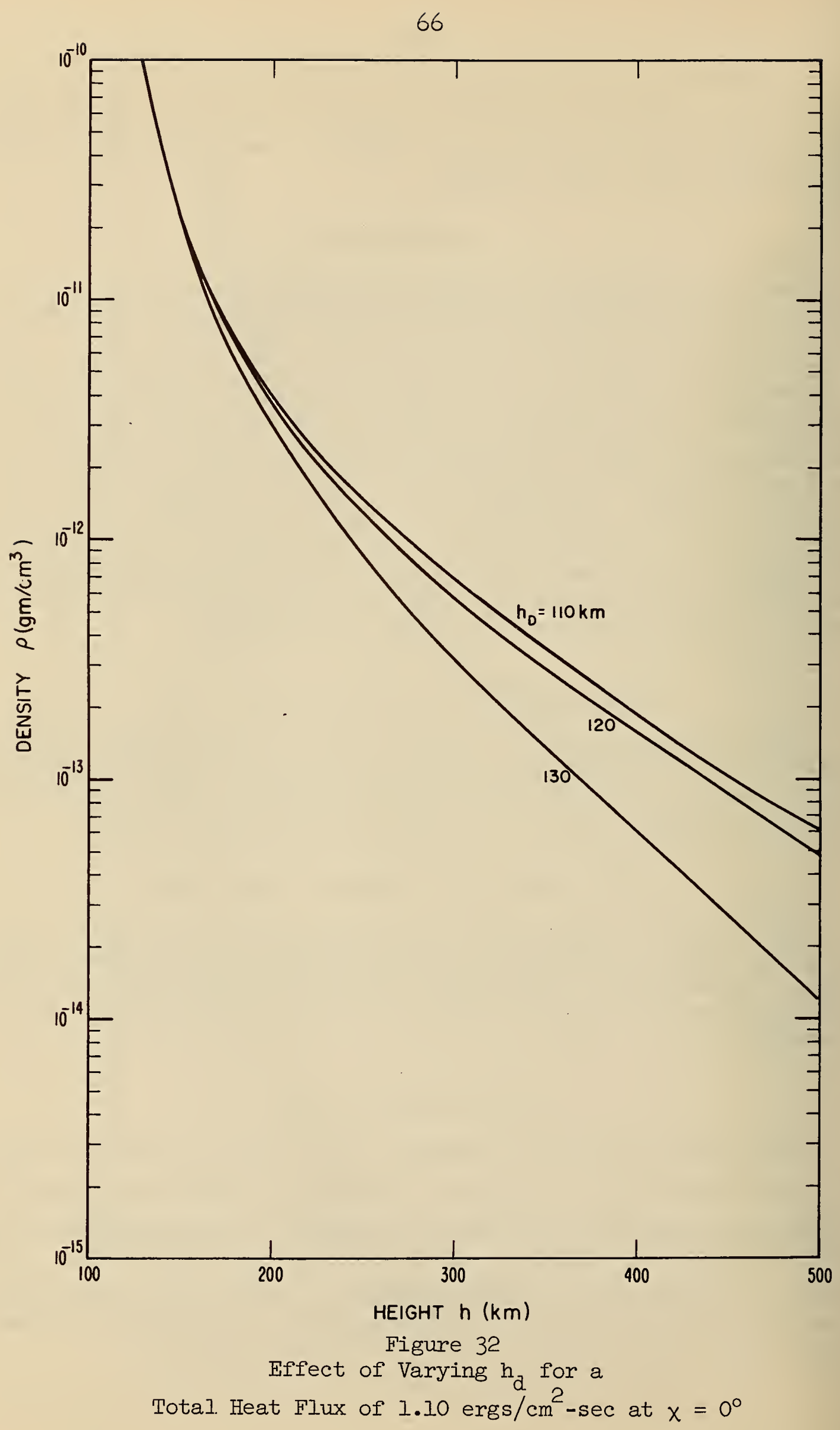




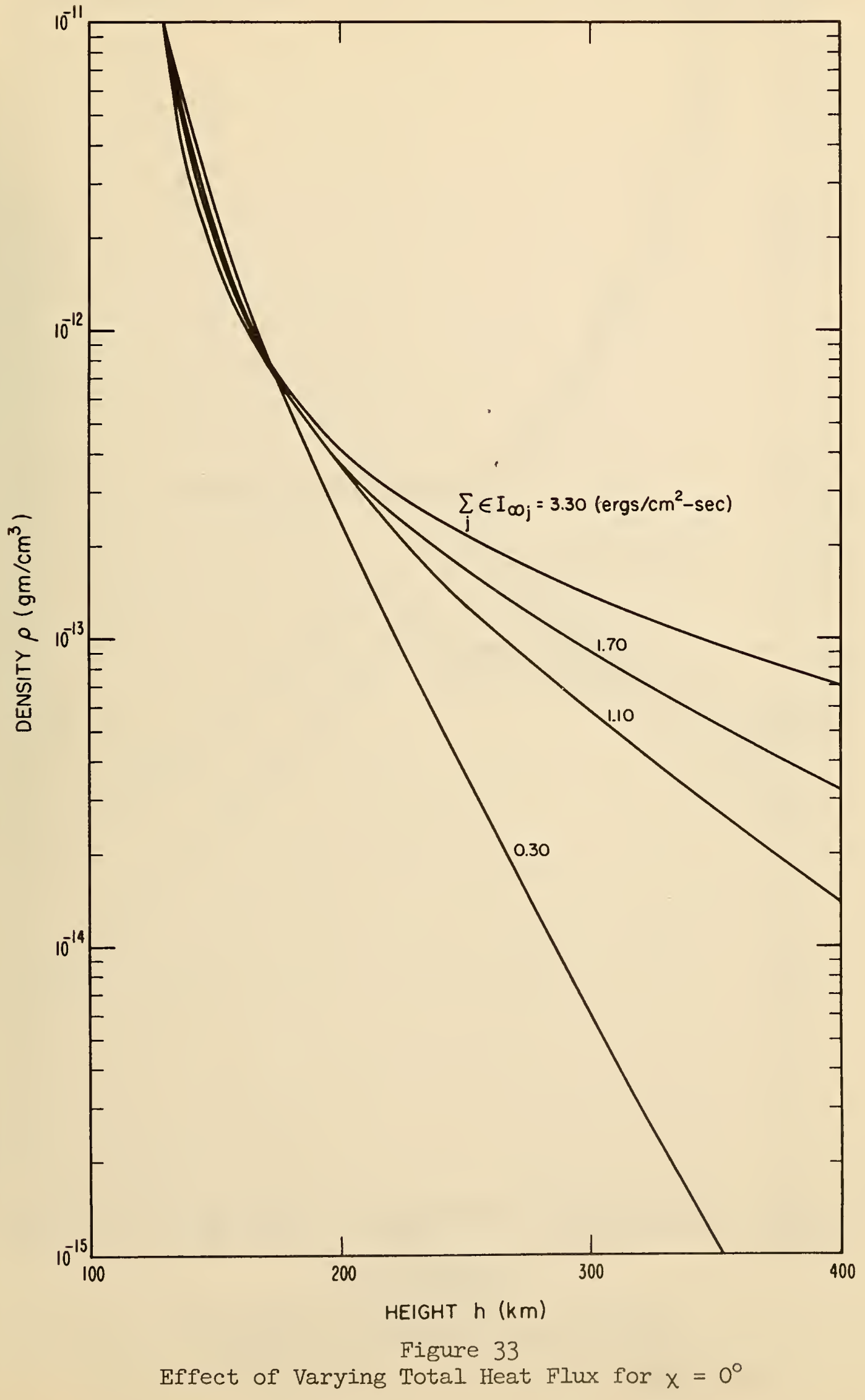




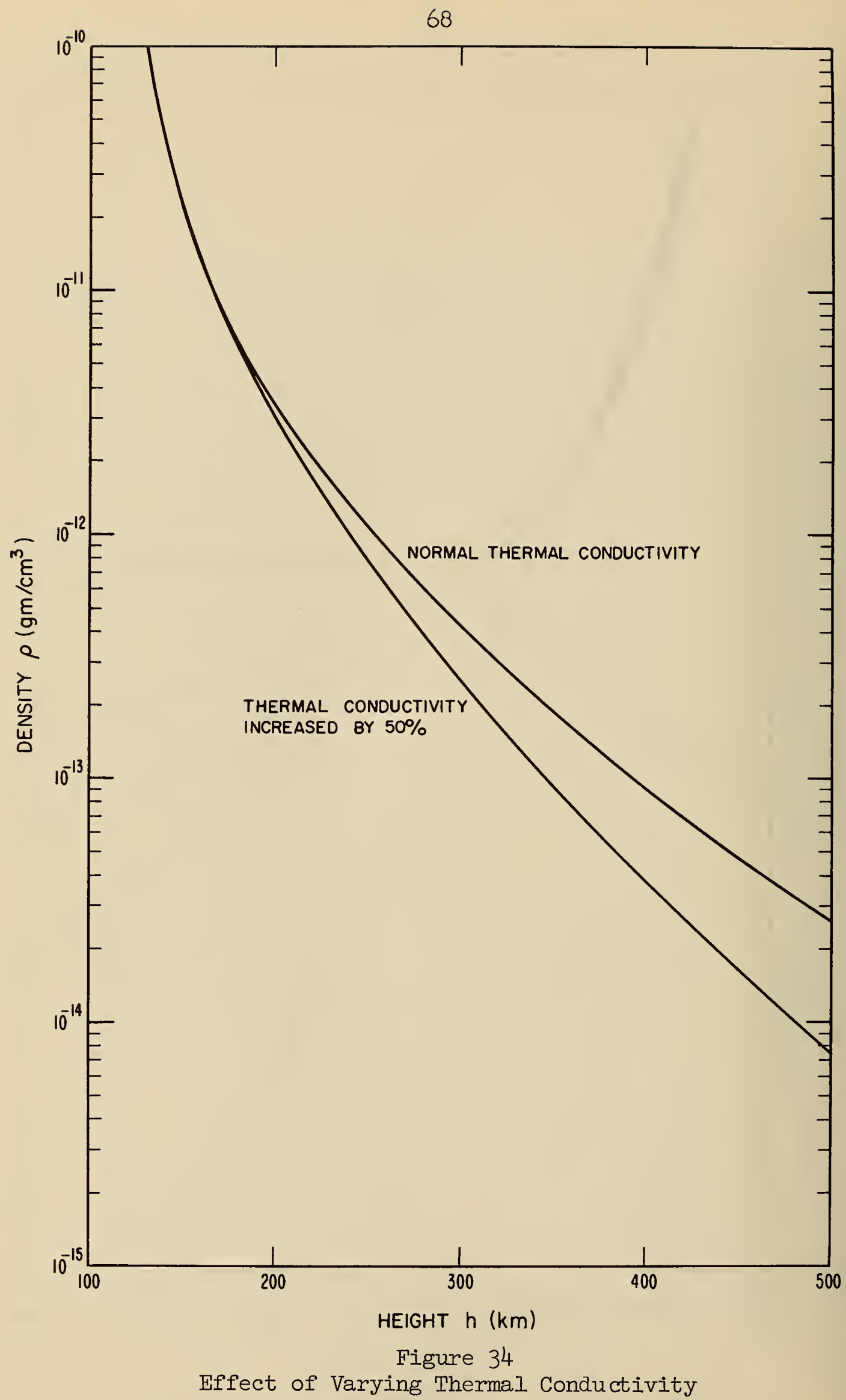

For a Total Heat Flux of $1.1 \mathrm{ergs} / \mathrm{cm}^{2}-\mathrm{sec}$ at $x=0^{\circ}$ 
69

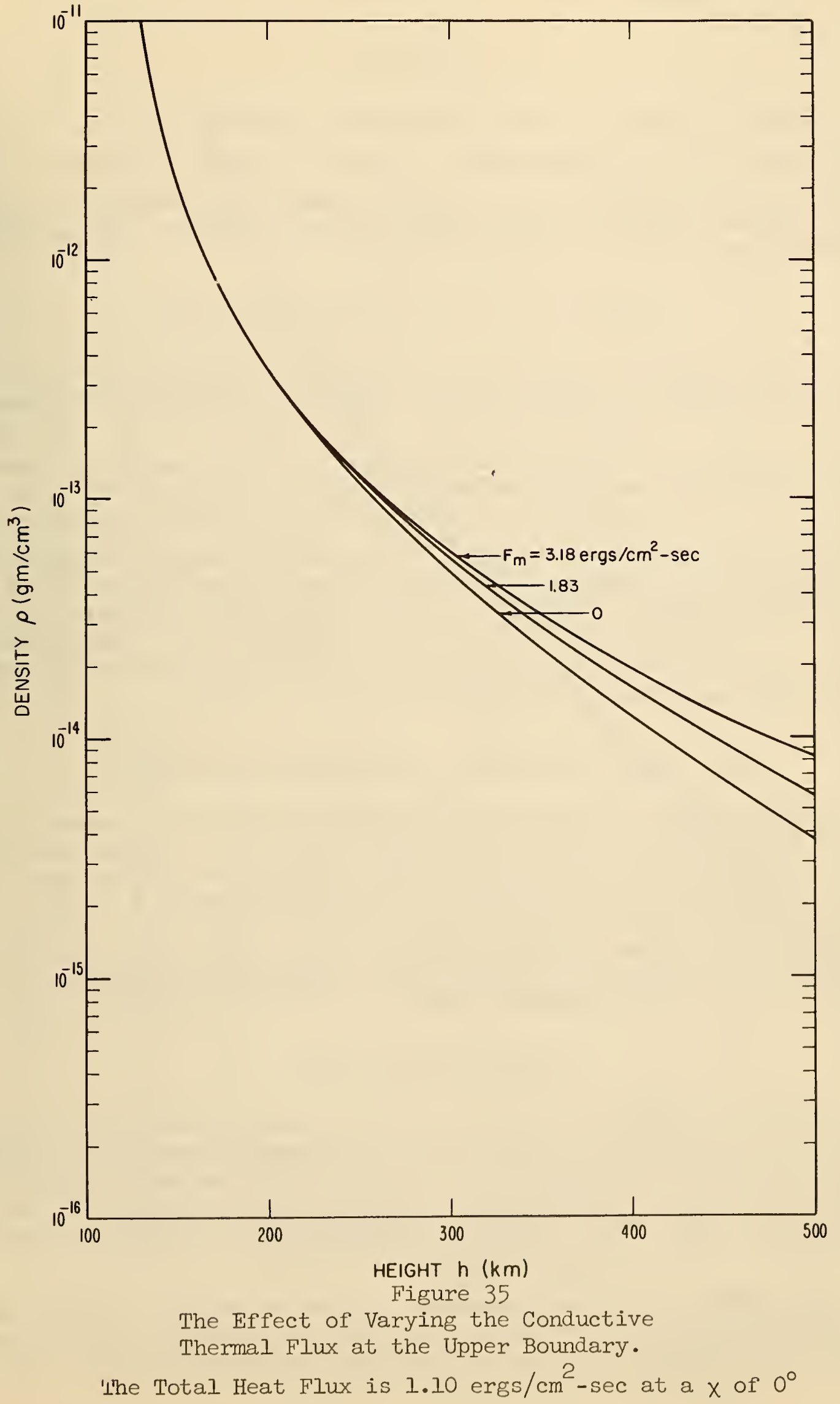


h. Composition

Figure 36 shows the total mass density profiles for pure $0, \mathrm{O}_{2}$, an $\mathrm{N}_{2}$ atmospheres. The temperature is adjusted so that the boundary scale height is always $15 \mathrm{~km}$. It is seen by comparing the observational density profile in figure 10 that atomic oxygen is the best approximation to the thermal situation in the F-region.

\section{i. Blamont, et al. (1961) Boundary Conditions}

If the previously mentioned boundary temperatures of $350^{\circ} \mathrm{K}$ is taken, a diffusive height of about $110 \mathrm{~km}$ along with 100 percent dissociation of $\mathrm{O}_{2}$ is needed to keep the boundary scale height at $15 \mathrm{~km}$. This is a case of varying $\mathrm{T} / \mathrm{M}$ together so that their quotient remains the same. The total density and detailed composition are plotted in figure 37. Such low $h_{d}$ and such a high degree of $\mathrm{O}_{2}$ dissociation indicate that a temperature of $350^{\circ} \mathrm{K}$ is probably somewhat low. Thus the detailed composition according to this boundary temperature is taken as an extreme model with the correct detailed composition closer to the model selected in this paper. If however, the upper range of the Blamont, et al. temperature at 130 is used $\left(395^{\circ} \mathrm{K}\right.$ ) and if an $\mathrm{h}_{\mathrm{d}}$ of $100 \mathrm{~km}$ is taken, a composition model may be made which has a sufficient amount of $\mathrm{O}_{2}$ in it.

\subsection{Range of Validity of Quasi-steady State Model}

On this model the afternoon and morning densities and temperatures at a fixed height are symmetric about noon $\left(x=0^{\circ}\right)$. However, satellite observations (Martin and Priester, 1960) indicate the diurnal mass variation to be symmetric about noon at low heights and about $x_{0}=30^{\circ}$ at high heights. Thus if the zero of $x$ were shifted to about $+20^{\circ}$, the agreement with observation would be quite good to about $x= \pm 50^{\circ}$ (see figure 11 ). Beyond this, the quasi-steady state drops considerably below the observed densities due to the increasing importance of the heat capacity term.

\subsection{The Non-steady State}

As seen in figure 26, there is rough agreement between the form of the observational density-versus- $x$ and the computed form. The computed curve has too large an amplitude and the amplitude maxima are generally $20^{\circ}-30^{\circ}$ too late in the afternoon. However, this discrepancy is due in large part to the assumption that the quasi-steady state holds at noon and not at $x=20^{\circ}$, as the observations indicate.

The complete solution to the heat balance equation, including the heat capacity term, can probably closely approximate the entire diurnal density and temperature variation as the following qualitative discussion 


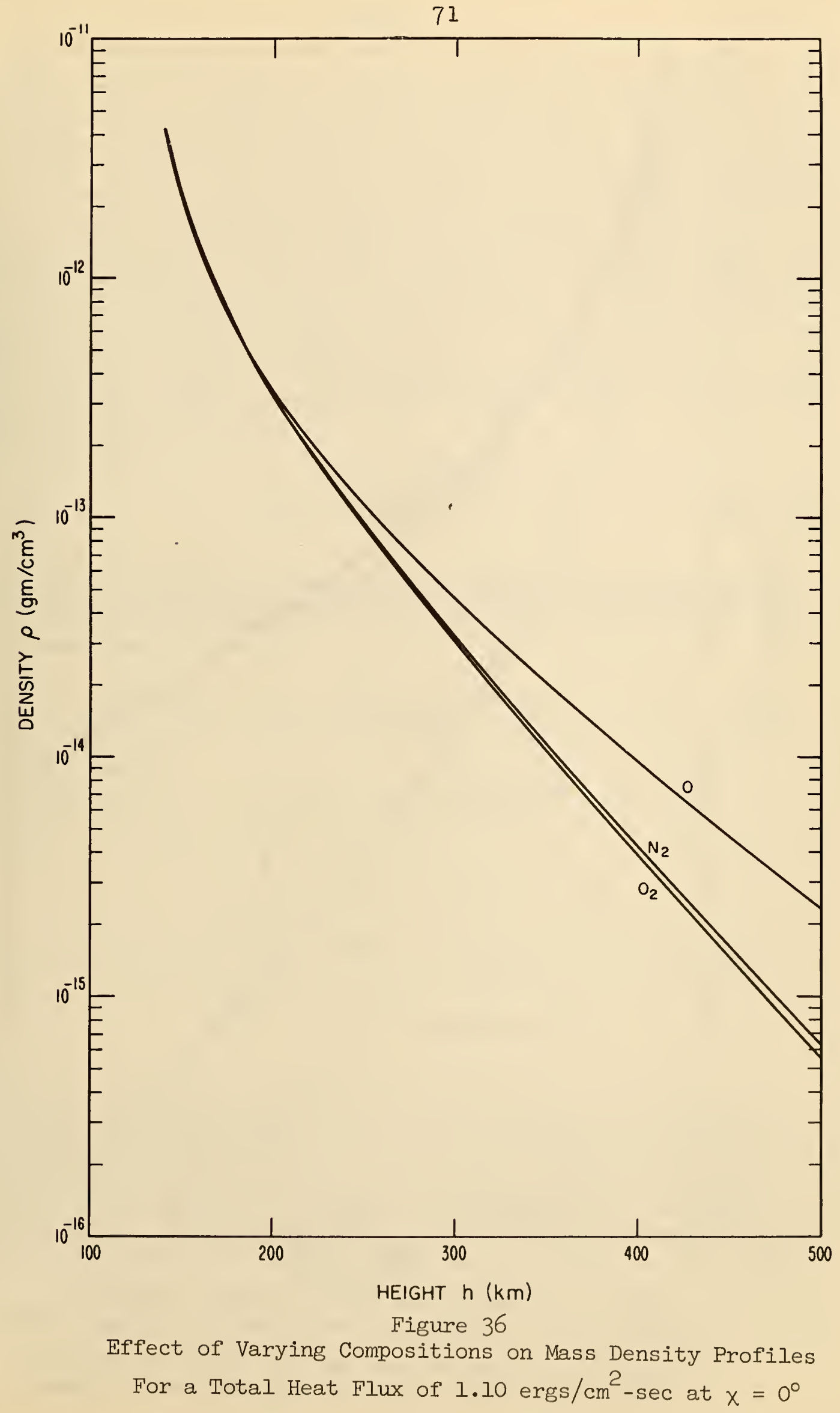




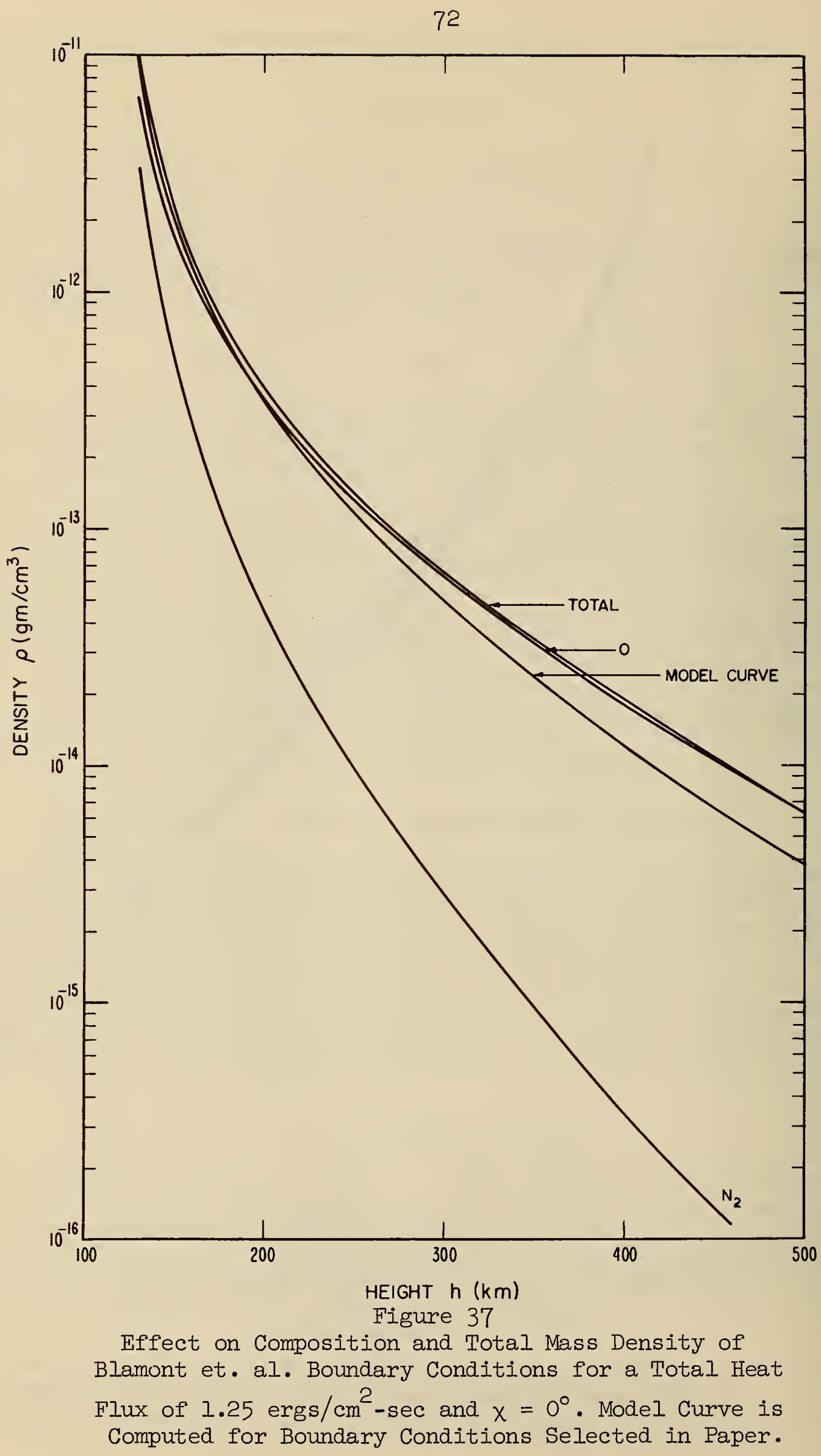


indicates. The heat capacity term is a measure of the sluggishness of the atmosphere in responding to thermal influences. It tends to oppose any atmospheric temperature changes. Thus the moming temperatures and densities will not be able to rise as rapidly as they would according to the quasi-steady state and will peak later than noon, as is observed. Also the afternoon temperature drop will be inhibited giving rise to higher densities at large $X^{\prime}$ s than those predicted by the quasi-steady state. The nighttime temperature drop will be likewise lessened by the heat capacity although isothermality will extend probably as low as $200-250 \mathrm{~km}$ by the night's end (Nicolet, 196I). If the total heat capacity of the atmosphere and the average conductive flux is estimated, it may be seen that the nighttime F-region atmosphere cannot be cooled on the average by more than about $800^{\circ} \mathrm{K}$ in twelve hours through conductive loss. This is seen by using the equation

$$
\Delta t=\overline{\rho c} \overline{\mathrm{H}}_{\Delta \mathrm{T}} \overline{\mathrm{KT}^{\mathrm{I} / 2}} \overline{\mathrm{T}^{\prime}} .
$$

Here $T^{\prime} \equiv \partial T / \partial h$ and the bars mean averages of the indicated quantities over the height ranges in which they are important. The assumed values are:

$$
\begin{aligned}
\bar{\rho} & =10^{-12} \mathrm{gms} / \mathrm{cm}^{3} \\
\bar{c}_{\mathrm{v}} & =8.3 \times 10^{6} \mathrm{ergs} / \mathrm{gm}^{\circ} \mathrm{K} \\
\overline{\mathrm{H}} & =40 \mathrm{~km} \\
\overline{\mathrm{T}^{\prime}} & =5^{\circ} \mathrm{K} / \mathrm{km} \\
\mathrm{KT}^{I / 2} & =6 \times 10^{4} \mathrm{ergs} / \mathrm{sec}-\mathrm{cm}^{\circ}{ }^{\circ} \mathrm{K} .
\end{aligned}
$$

According to (5-1) it will take about 25 hours for the atmosphere to cool $800^{\circ} \mathrm{K}$. Taking average radiative loss values increases the denominator of $(5-1)$ by about 10 percent and so decreases the cooling time by about the same amount. Observations (figure 9) show the nighttime scale heights to be about 60 percent of the daytime scale heights from 400 to $500 \mathrm{~km}$. A decrease from about $1700^{\circ} \mathrm{K}$ (predicted by the quasi-steady state model) to about $900^{\circ} \mathrm{K}$ would indicate a similar percentage decrease in the scale height. 
5.5 The Structure in the Observational Density and Scale Height Profiles

An anomaly is seen in the observational mass density and scale height curves (figures 9 and 10) at about $200 \mathrm{~km}$. This anomaly shows up as a pair of inflection points in the mass density curve and as an inversion in the scale height curve. A scale height inversion will almost certainly imply a negative T'. For this to occur (2-10-2) shows that:

$$
\int_{\angle O C}^{h}\left(c_{v} \rho \dot{T}+L\right) d h \geqq \int_{200}^{h} G d h .
$$

On the theory as used here it is difficult to get an inversion in the temperature except possibly near sunrise or late in the night. However, (5-2) could not be satisfied all day and all night as the observations indicate.

There are several possible explanations. Johnson (1960) has pointed out the possibility of time dependent heat convection from the polar regions at about $200 \mathrm{~km}$. This would cause a sharp temperature rise. Then at slightly higher heights, where the convective flux stops, the values of $c_{v} \rho T$ and $I$ could be high enough to satisfy (5-2). Such a convective flow could lead to sharp gradients in $\vec{c}_{0}$, which have been neglected in the heat balance equation. These would have the effect (if positive) of increasing the Ieft hand side of (5-2). Another possibility is that the charged particle conduction could be high here due to a high electron temperature (caused by low electron densities and the input of many high energy electrons near $200 \mathrm{~km}$ ). Thus a cool spot could be created which would account for the observed inversion. It is, however, unlikely that electron temperatures high enough for this to occur $\left(\approx 10,000^{\circ} \mathrm{K}\right)$ could actually exist in the F-region. It can be asserted that whatever the mechanism it is not due to differential absorption of ultraviolet flux.

\subsection{Conclusions}

The primary conclusion is that ultraviolet photons are a major Fregion heating source. The necessary sunspot maximum heat flux is found to be 1.13 ergs $/ \mathrm{cm}^{2}-\mathrm{sec}$, giving an estimated photon energy flux of 5.18 ergs $/ \mathrm{cm}^{2}-\mathrm{sec}$. The estimated error (due mainly to the uncertainty in the $\mathrm{T} / \mathrm{M}$ ratio at $130 \mathrm{~km}$ ) is about \pm 30 percent. This compares favorably with the extrapolation of Hinteregger's (1960) measured ultraviolet flux values made by Denison (1961), who estimates $5.66 \mathrm{ergs} / \mathrm{cm}^{2}-\mathrm{sec}$ in the Fregion ionizing flux. 
The non-steady state heat balance equation can qualitatively explain the diurmal variation of the F-region mass densities and temperatures during the aftermoon. The quasi-steady state quantitatively explains the F-region diurnal variation of mass densities and temperature for $x \leqq 50^{\circ}$ and up to about $300 \mathrm{~km}$. The detailed composition models and ultraviolet flux absorption parameter models are valid over the same ranges $\left(x \leqq 50^{\circ}, \mathrm{h} \leqq 300 \mathrm{~km}\right)$.

The uncertainties in the parameters and boundary conditions necessary in the theory are insufficiently large to invalidate the above conclusions.

\subsection{Future Studies Indicated}

The complete solution of the non-steady state for daytime and nighttime is the most important extension of the present work. The method of solution attempted in the present study could be improved by a more accurate numerical calculation of $T$ and better boundary conditions. When the non-steady state problem is completely solved, seasonal and latitudinal variations may be investigated through the transformation of $T$ to $\partial T / \partial x$

The use of the heat balance equation (with appropriate extra gain terms) to explain the critical points in the density and scale height curves near $200 \mathrm{~km}$ is a study which should be done. The magnitude of the density inflection and the scale height inversion is a measure of the strength of the necessary gain terms.

The heat balance equation may also be used to study atmospheres of other planets. For example, the Martian atmosphere is thought to be mostly $N_{2}$. Also, the force of gravity on Mars is less than on Earth and the solar flux is less at Mars than at Earth. Appropriate boundary conditions are not known but may be roughly estimated from the previous facts. This is all the information necessary to apply the heat balance equation. 


\section{ACKNOWLEDGMENTIS}

The author wishes to express his gratitude to his wife for aid and encouragement, to $\mathrm{Dr}$. T. E. VanZandt for many valuable consultations and helpful criticisms, and to $\mathrm{Mr}$. D. L. Obitts for technical advice on computer programming. Also, the author is grateful to both the National Science Foundation and to the National Bureau of Standards for partial support of this work. 


\section{BIBLIOGRAPHI}

Bates, D. R. and M. J. Seaton, "The Quantal Theory of Continuous Absorption of Radiation by Various Atoms in Their Ground States," Mon. Not. Roy. Astron. Soc., 109, 698 (1949).

Bates D. R., "The Temperature of the Upper Atmosphere," Proc. Phys. Soc. (Iondon), B 64,805 (1951).

Bates, D. R., "Some Problems Concerning the Terrestrial Atmosphere Above the $100 \mathrm{~km}$ Level," Proc. Roy. Soc., A 253, 456 (1959).

Blamont, J. E., T. M. Donahue, and M. L. Lory, "Measurement of the Temperature in the Upper Atmosphere to $150 \mathrm{~km}$ in a Rocket Experiment," Phys. Rev. Letters, 6, No. 8, 403 (1961).

Brokaw, R. S., "Energy Transport in High Temperature and Reacting Gases," Planetary and Space Sci., $\underline{3}, 238$ (1961).

Carslaw, H. S. and J. C. Jaeger, Conduction of Heat in Solids, p. 243, (Iondon, Oxford University Press, 1959).

Chapman, S., "Notes on the Solar Corona and the Terrestrial Ionosphere," Smithsonian Contribs. to Astrophys., 2 , I (1957).

Chapman, S. and T. G. Cowling, The Mathematical Theory of Non-Uniferm Gases, p. 241 (Iondon, Cambridge University Press, 1952a).

Chapman, S. and T. G. Cowling, The Mathematical Theory of Non-Uniform Gases, p. 107 (Iondon, Cambridge University Press, 1952b).

Chapman, S. and T. G. Cowling, The Mathematical Theory of Non-Uniform Gases, p. 169 (Iondon, Cambridge University Press, 1952c).

Chapman, S. and T. G. Cowling, The Mathematical Theory of Non-Uniform Gases, p. 166 (Iondon, Cambridge University Press, 1952d).

Chapman, S. and"T. G. Cowling, The Mathematical Theory of Non-Uniform Gases, p. 179 (Iondon, Cambridge University Press, 1952e).

Dalgarmo, A. and D. Parkinson, "Photoionization of Atomic Oxygen and Atomic Nitrogen," J. Atmospheric and Terrest. Phys., 18, 335 (1960).

Daniels, F. B., "Acoustical Energy Generated by the Ocean Waves," J. Acoust. Soc. Am., 24, 83 (1952). 
Denison, Judith S., Predicted Photon Fluxes and Photoionization Rates in the Upper Atmosphere, Master's Thesis, University of Colorado (1961).

Dessler, A. J., "Ionospheric Heating by Hydromagnetic Waves," J. Geophys. Research, 64, 397 (1959).

Friedman, H., "The Solar Spectrum Below 2000 A," Ann. Geophys., 11, 174 (1955).

Friedman, H., "The Sun's Ionizing Radiation", Physics of the Upper Atmosphere, p. 168 (New York, Academic Press, 1960).

Hanson, W. B. and F. S. Johnson, "Electron Temperatures in the Ionosphere, Memoires R. Sc. Soc. Tiege, Fifth Series, 4, 390 (1961).

Hinteregger, H. E., "Interplanetary Ionization by Solar Extreme UItraviolet Radiation," Astrophys. J., 132, 801 (1960).

Hunt, D. C. and T. E. Vanzandt, "Photoionization Heating in the F-Region of the Ionosphere," J. Geophys. Research, 66, 1673 (1961).

Johnson, F. S., J. D. Purcell, and R. Tousey, "Measurements of the Vertical Distribution of Atmospheric Ozone," J. Geophys. Research, 56, 583 (1951).

Johnson, F. S., "Temperatures in the High Atmosphere," Ann. Geophys., 14, 94 (1958).

Kaiser, T. R., "Meteors," J. Atmos. and Terrest. Phys., Suppl. 2, 119 (1955).

Kallmann-Bijl, H. K., "Daytime and Nighttime Atmospheric Properties Derived from Rocket and Satellite Observations," J. Geophys. Research, 66, 787 (1961).

Kallmann, H. Korf, "Upper Atmosphere Properties Based on Rocket and Satellite Data," Physics and Medicine of the Atmosphere and Space, p. 100 (Editëd by Benson and Strughold, Wiley Press, 1960).

Keyes, F. G., "The Heat Conductivity, Viscosity, Specific Heat, and Prandtl Numbers for Thirteen Gases," M. I. T. Technical Report 37, Project Squid, 1952 .

Krassovsky, V. I., "Fnergy Sources of the Upper Atmosphere," Planetary and Space Sci., 1, 19 (1959). 
Krassovsky, V. I., "Corpuscular Heating of the Outer Atmosphere," Space Research, p. 90 (Edited by H. Kallmann-Bijl, North Holland Publishing Co., Amsterdam, 1960).

Kuiper, G. P., The Atmosphere of the Farth and Planets, p. 312 (Chicago, University of Chicago Press, 1952).

Kupperian, J. E., Jr., E. T. Byram, and H. Friedman, "Molecular Oxygen Densities in the Mesosphere at Fort Churchill," J. Atmospheric and Terrest. Phys., 16, 174 (1959).

Lovell, A. C. B., and J. S. Greenhow, "The Upper Atmosphere and Meteors," Physics of the Upper Atmosphere, p. 513 (Edited by J. A. Ratcliffe, Academic Press, New York, 1960).

Martin, H. A. and W. Priester, "Measurement of Solar and Diurnal Effects in the High Atmosphere by Artificial 'Satellites," Nature, 185, 600 (1960).

McIlwain, C. E., "Direct Measurements of Particles Producing Visible Auroras," J. Geophys. Research, 65, 2727 (1960).

Meadows, E. B. and J. W. Townsend, Jr., "I. G. Y. Rocket Measurements of Arctic Atmosphere Composition Above $100 \mathrm{~km}$," Space Research, p. 175 (Edited by H. Kallmann-Bijl, North Holland Publishing Co., Amsterdam, 1960).

Minzner, R. A., K. S. W. Champion, H. I. Pond, "The ARDC Model Atmosphere, 1959, "Air Force Cambridge Research Center Technical Report, 59-267, 1959.

Mitra, S. K., The Upper Atmosphere, p. 24 (The Asiatic Society, Calcutta 1952 ).

Nicolet, M., "Sturcture of the Thermosphere," Planetary and Space Sci., 5, I (1961).

Nicolet, M., "The Properties and Constitution of the Upper Atmosphere," Physics of the Upper Atmosphere, p. 37 (Academic Press, New York, 1960).

Nicolet, M. and P. Mange, "The Dissociation of Oxygen in the High Atmosphere," J. Geophys. Research, 59, 15 (1954).

Penndorf, R., "The Vertical Distribution of Atomic Oxygen in the Upper Atmosphere," J. Geophys. Research, 54, 7 (1949). 
Sugiura, M., "Some Evidence of Hydromagnetic Waves in the Earth's Magnetic Field," Phys. Rev. Letters 6, 255 (1961).

Victoreen, J. A., "The Calculation of X-ray Mass Absorption Coefficients, J. Applied Phys., 20, 1.141 (1949).

Violett, T. and W. A. Rense, "Solar Emission Lines in the Extreme Ultraviolet," Astrophys. J., 130, 954 (1959).

Wulf, O. R. and I. S. Deming, "The Distribution of Atmospheric Ozone in Equilibrium with Solar Radiation and the Maintenance of the Distribution," Terrest. Magnetism and Atmos. Electricity, 42, 195 (1937). 


\section{U. S. DEPARTMENT OF COMMERCE}

Luther H. Hodges, Secretary

NATIONAL BUREAU OF STANDARDS

A. V. Astin, Director

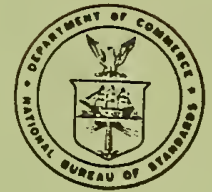

\section{THE NATIONAL BUREAU OF STANDARDS}

The scope of activities of the National Bureau of Standards at its major laboratories in Washington, D.C., and Boulder, Colorado, is suggested in the following listing of the divisions and sectionsengaged in technical work. In general, each section carries at specialized research, development, and engineering in the field indicated by its title. A brief description of the activities, and of the resultant publications, appears on the inside of the front cover.

\section{WASIIINGTON, D. C.}

Electricity. Resistance and Reactance. Electrochemistry. Electrical Instruments. Magnetic Measurements Dielectrics. High Voltage.

Metrology. Photometry and Colorimetry. Refractometry. Photographic Research. Length. Engineering Metrology. Mass and Scale. Volumetry and Densimetry.

Heat. Temperature Physics. Heat Measurements. Cryogenic Physics. Equation of State. Statistical Physics. Radiation Physics. X-ray. Radioactivity. Radiation Theory. High Energy Radiation. Radiological Equipment. Nucleonic Instrumentation. Neutron Physics.

Analytical and Inorganic Chemistry. Pure Subst ances. Spectrochemistry. Solution Chemistry. StandardReference Materials. Applied Analytical Research. Crystal Chemistry.

Mechanics. Sound. Pressure and Vacuum. Fluid Mechanics. Engineering Mechanics. Rheology. Combustion Controls.

Polymers. Macromolecules: Synthesis and Structure. Polymer Chemistry. Polymer Physics. Polymer Characterization. Polymer Evaluation and Testing. Applied Polymer Standards and Research. Dental Research.

Metallurgy. Engineering Metallurgy. Microscopy and Diffraction. Metal Reactions. Metal Physics. Electrolysis and Metal Deposition.

Inorganic Solids. Engineering Ceramics. Glass. Solid State Chemistry. Crystal Growth. Physical Properties. Crystallography.

Building Research. Structural Engineering. Fire Research. Mechanical Systems. Organic Building Materials. Codes and Safety Standards. Heat Transfer. Inorganic Building Materials. Metallic Building Materials.

Applied Mathematics. Numerical Analysis. Computation. Statistical Engineering. Mathematical Physics. Operations Research.

Data Processing Systems. Components and Techniques. Computer Technology. Measurements Automation. Engineering Applications. Systems Analysis.

Atomic Physics. Spectroscopy. Infrared Spectroscopy. Far Ultraviolet Physics. Solid State Physics. Electron Physics. Atomic Physics. Plasma Spectroscopy.

Instrumentation. Engineering Electronics. Electron Devices. Electronic Instrumentation. Mechanical Instruments. Basic lnstrumentation.

Physical Chemistry. Thermochemistry. Surface Chemistry. Organic Chemistry. Molecular Spectroscopy. Elementary Processes. Mass Spectrometry. Photochemistry and Radiation Chemistry.

Office of Weights and Measures.

BOULDER, COLO.

Cryogenic Engineering Laboratory. Cryogenic Equipment. Cryogenic Processes. Properties of Materials. Cryogenic Technical Services.

\section{CENTRAL. RADIO PROPAGATION LABORATORY}

Ionosphere Research and Propagation. Low Frequency and Very Low Frequency Research. Ionosphere Research. Prediction Services. Sun-Earth Relationships. Field Engineering. Kadio Warning Services. Vertical Soundings Research.

Radio Propagation Engineering. Data Reduction Instrumentation. Radio Noise. Tropospheric Measurements. Tropospheric Analysis. Propagation-Terrain Effects. Radio-Meteorology. Lower Atmosphere Physics.

Radio Systems. Applied Electromagnetic Theory. High Frequency and Very High Frequency Research. Frequency Utilization. Modulation Research. Antenna Research. Radiodetermination.

Upper Atmosphere and Space Physics. Upper Atmosphere and Plasma Physics. High Latitude Ionosphere Physics. lonosphere and Exosphere Scatter. Airglow and Aurora. Ionospheric Kadio Astronomy.

\section{RADIO STANDARDS LABORATORY}

Radio Physics. Radio Broadcast Service. Radio and Microwave Materials. Atomic Frequency and Time-Interval Standards. Kadio Plasma. Millimeter-Wave Research.

Circuit Standards. High Frequency Electrical Standards. High Frequency Calibration Services. Iligh Frequency Impedance Standards. Microwave Calibration Services. Microwave Circuit Standards. Low Frequency Calibration Services. 
NBS 